\title{
Vestibular exploration : on advanced diagnostics and therapy
}

Citation for published version (APA):

Janssen, M. J. A. (2011). Vestibular exploration : on advanced diagnostics and therapy. [Doctoral Thesis, Maastricht University]. Maastricht University. https://doi.org/10.26481/dis.20110706mj

Document status and date:

Published: 01/01/2011

DOI:

10.26481/dis.20110706mj

Document Version:

Publisher's PDF, also known as Version of record

\section{Please check the document version of this publication:}

- A submitted manuscript is the version of the article upon submission and before peer-review. There can be important differences between the submitted version and the official published version of record.

People interested in the research are advised to contact the author for the final version of the publication, or visit the DOI to the publisher's website.

- The final author version and the galley proof are versions of the publication after peer review.

- The final published version features the final layout of the paper including the volume, issue and page numbers.

Link to publication

\footnotetext{
General rights rights.

- You may freely distribute the URL identifying the publication in the public portal. please follow below link for the End User Agreement:

www.umlib.nl/taverne-license

Take down policy

If you believe that this document breaches copyright please contact us at:

repository@maastrichtuniversity.nl

providing details and we will investigate your claim.
}

Copyright and moral rights for the publications made accessible in the public portal are retained by the authors and/or other copyright owners and it is a condition of accessing publications that users recognise and abide by the legal requirements associated with these

- Users may download and print one copy of any publication from the public portal for the purpose of private study or research.

- You may not further distribute the material or use it for any profit-making activity or commercial gain

If the publication is distributed under the terms of Article $25 \mathrm{fa}$ of the Dutch Copyright Act, indicated by the "Taverne" license above, 
Vestibular Exploration ON ADVANCED DIAGNOSTICS AND THERAPY 
Printing of this thesis was financially supported by

Maastricht Instruments bv

School of Medical Physics and Engineering Eindhoven

Cover design, printed and published by proefschriftmaken.nl

ISBN 978-90-8891-259-7

(C) Maurice Janssen, Spaubeek 2011 


\section{Vestibular Exploration}

ON ADVANCED DIAGNOSTICS AND THERAPY

\section{PROEFSCHRIFT}

ter verkrijging van de graad van doctor aan de Universiteit Maastricht, op gezag van de Rector Magnificus, prof. mr. G.P.M.F. Mols, volgens het besluit van het College van Decanen, in het openbaar te verdedigen op woensdag 6 juli 2011 om 16.00 uur

door

Maurice Joseph Antoon Janssen 


\section{Promotores:}

prof. dr. Herman Kingma

prof. dr. Robert Stokroos

\section{Copromotor:}

dr. ir. Jos Reulen

Beoordelingscommissie:

prof. dr. Bernd Kremer (voorziter)

prof. dr. Måns Magnusson (Lund University, Sweden)

dr. Jaap Patijn

prof. dr. Floris Wuyts (Universiteit van Antwerpen, België) 


\section{Contents}

Part A: General Introduction

Part B: Control of Posture

Chapter 1: Effectiveness of vibrotactile biofeedback in healthy subjects on a tilting platform

Chapter 2: Clinical observational gait analysis to evaluate improvement of balance during gait with vibrotactile biofeedback

Chapter 3: Salient and placebo vibrotactile feedback are equally effective in reducing sway in bilateral vestibular loss patients

Part C: Spatial Orientation

Chapter 4: Clinical application of perception threshold of horizontal plane rotation

Chapter 5: Thresholds of tilt and translation perception in healthy subjects

Part D: Eye Movement Quantification

Chapter 6: Measuring saccade peak velocity using a low-frequency sampling rate of $50 \mathrm{~Hz}$

Chapter 7: Quantitative and qualitative comparison of electro nystagmography (ENG), video oculography (VOG) and scleral search coil (SSC) in measuring horizontal saccades

Part E: Summary and Concluding Remarks

Scientific Output

Samenvatting

Nawoord

Curriculum Vitae

Stellingen 


\section{Part A}

GENERAL INTRODUCTION 
Part A

This thesis would not have been possible without the support from Herman Kingma, Jos Reulen and Robert Stokroos and the technicians Ellen Rikers, Sophie Paredis and Marie-Cécile Gerards from the vestibular department. 
Head movement and orientation are sensed by two vestibular organs of the inner ear, supported by other sensory systems. Both vestibular organs are predominantly sensitive for accelerations. They both contain two otolith organs, which detect linear accelerations, and three semicircular canals (scc), which detect angular accelerations. As schematically shown in figure A.1, the three semicircular canals (lateral, anterior, and posterior) are oriented almost mutually perpendicular. The lateral (horizontal) canal is oriented under an angle of about $30^{\circ}$ with the transverse plane, while the anterior and posterior (vertical) canals are located vertically under an angle of about $45^{\circ}$ with the coronal plane [Huizing et al., 2007] and more or less perpendicular to the lateral canal. Each semicircular canal has a coplanar canal in the contralateral ear, so that any head rotation results in stimulation of at least two semicircular canals, a coplanar canal pair. The coplanar pairs are 1) left and right horizontal canal, 2) left posterior and right anterior canal and 3) left anterior and right posterior canal.

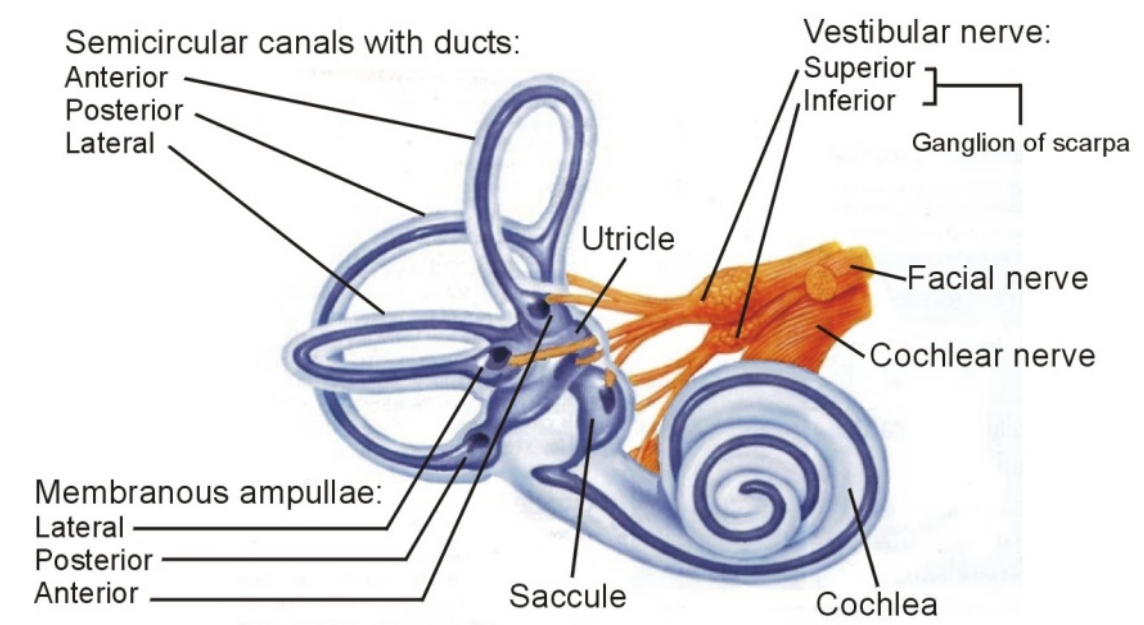

Figure A.1: Schematic representation of a vestibular organ and cochlea.

The two otolith organs are oriented almost mutually perpendicular as well, and allow detection of linear accelerations in two dimensions each. The utricule is oriented parallel to the transverse plane and is primarily sensitive for naso-occipital (for-aft) and interaural (left-right) accelerations. The saccule is oriented parallel to the sagittal plane and is primarily sensitive for naso-occipital and cranio-caudal (up-down) accelerations. Each otolith organ has a coplanar otolith organ in the contralateral ear, so that any linear acceleration results in stimulation of at least one coplanar otolith pair. Therefore both vestibular organs detect head rotation, translation and orientation with respect to 
gravity with 3 degrees of freedom. By integrating - anatomically, physiologically and functionally [Bringoux et al., 2003, Stolbkov and Orlov, 2009] - information from both sensory (vision, vestibular organs, proprioception ${ }^{1}$, mechanoreceptors [Bringoux et al., 2003]) and nonsensory (efferent copy, cognition, interpretation, re-weighting [Mahboobin et al., 2009] etc.) sources, the vestibular system is formed [Rader et al., 2009]. This system has three major properties [Huizing et al., 2007, Luxon et al., 2003], as illustrated in figure A.2:

- image stabilization; compensatory eye movements during fast head movements

- postural control; body stability

- spatial orientation; determination of self- and object-motion

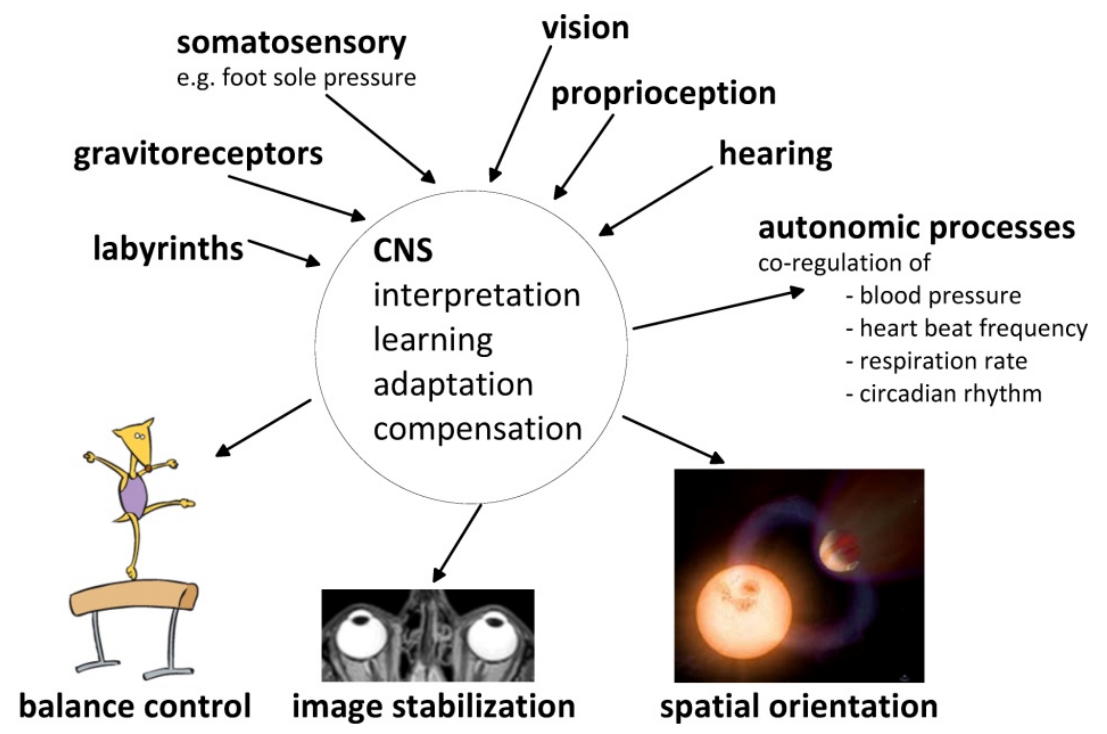

Figure A.2: Schematic representation of the multi-sensory control of image stabilization, balance control and spatial orientation. CNS = central nervous system

Despite some overlap in functionality, loss of any sensory system will reduce performance [Luxon et al., 2003]. Dysfunction of the vestibular organ thus leads to impairment in the three properties of the vestibular system.

Additionally, because of sensory overlap vestibular function tests still have limited sensitivity and specificity [Kingma, 2006, Merfeld et al., 2010].

\footnotetext{
${ }^{1}$ the capability to detect the position of your body parts [Oliver Sacks]
} 


\section{Theoretical background}

A vestibular organ contains three semicircular canals (scc), which detect angular accelerations, and two otolith organs, which detect linear accelerations. From both structures a theoretical background is discussed in this section.

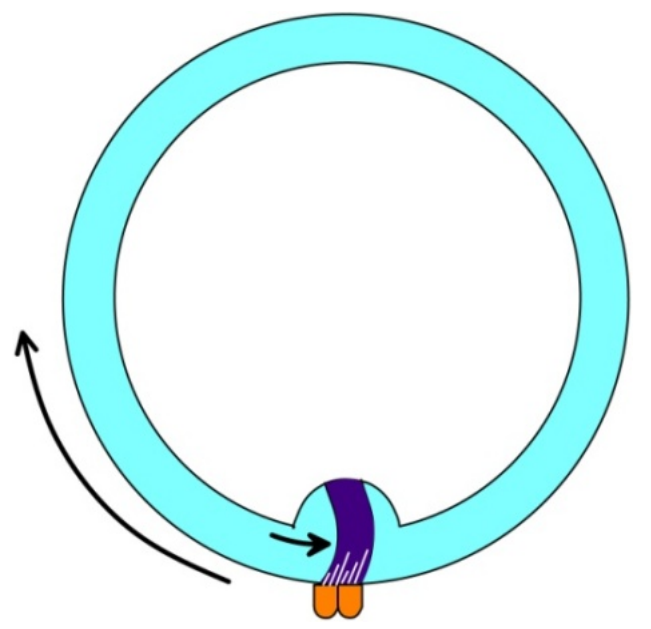

Figure A3: Schematic representation of the semicircular canal. The widened section represents the ampulla with the cupula and the hair cells at the base of the cupula

\section{Semicircular canals}

A schematic representation of a semicircular canal (scc) is shown in figure A.3. Each scc is filled with endolymph fluid, which is prevented from passing the ampulla (a widened section of each scc) by the cupula, a thin flap that stretches across the ampulla. When the head is rotated, the endolymph fluid lags behind due to mass inertia and exerts a force against the cupula of those scc's that are in the plane of motion (Ewald's first law states that the optimal rotation axis is perpendicular to a canal plane), causing the cupula to deflect/bend. This deflection causes a bending of the hair cells (the vestibular mechanoreceptors located at the base of the cupula) which signal this change to the brain via the $8^{\text {th }}$ nerve. Because all hair cells have the same polarization in the cupula, this bending causes depolarization or hyperpolarization of the hair cells depending on the rotation direction (with an asymmetric gain), which is known as Ewald's second law. Also, if one canal of the coplanar canal pair is depolarized, the other is hyperpolarized. This is called push-pull organization. 


\section{Compensatory eye rotation to the left}

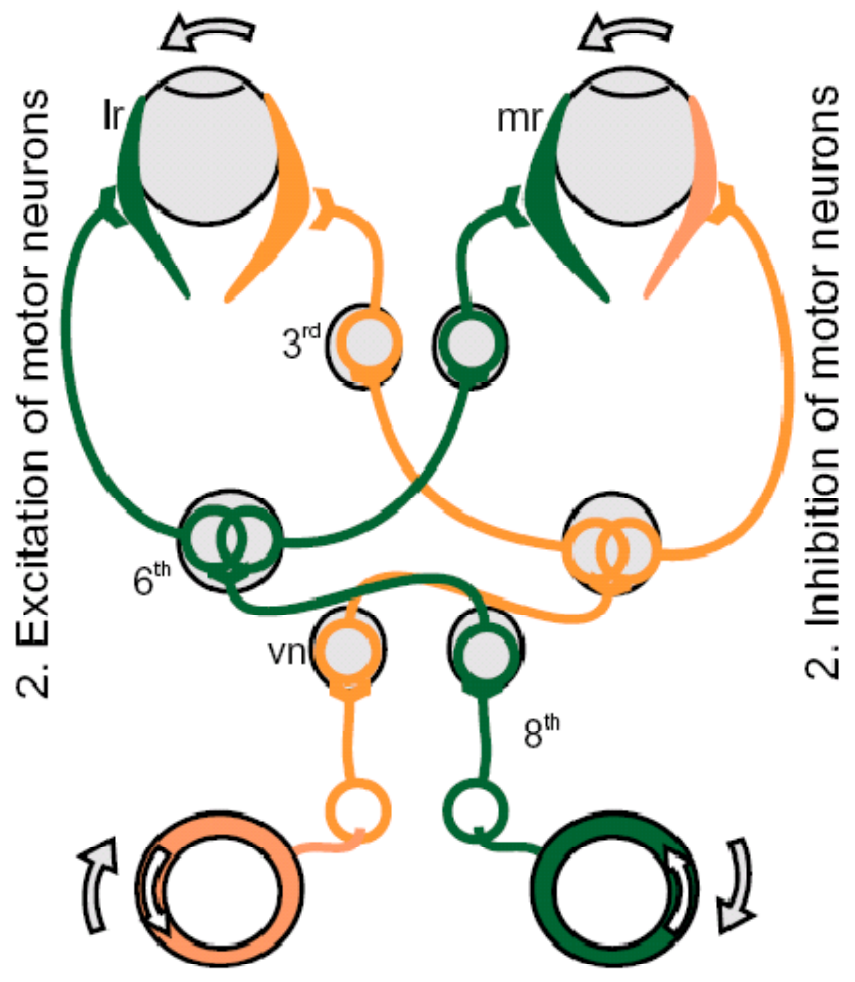

\section{Detect rotation to the right}

Figure A.4: Compensatory eye rotation to the left, induced by head rotation to the right. vn = vestibular nucleus, $I r=$ lateral rectus muscle (left), $m r=$ medial rectus muscle (right).

For example (figure A.4), when the head rotates rightward, depolarization occurs in the right horizontal canal and hyperpolarization occurs in the left. This results in an increase (decrease) of right (left) vestibular afferent and nucleus' activity and an increase (decrease) in left (right) oculomotor nucleus' activity. This results in contraction of the left and relaxation of the right extraocular muscles and thus in rotation of both eyes leftward, compensating for the head rotation to the right.

During head rotation the endolymph lags behind the movement of the scc due to mass inertia, causing viscous friction. Additionally, the deflected cupula has elastic properties. Therefore the scc can be modeled with a simple mechanical analogon, using inertia (I), viscosity (B) and elasticity/stiffness (K) as physical quantities, see figure A.5. 


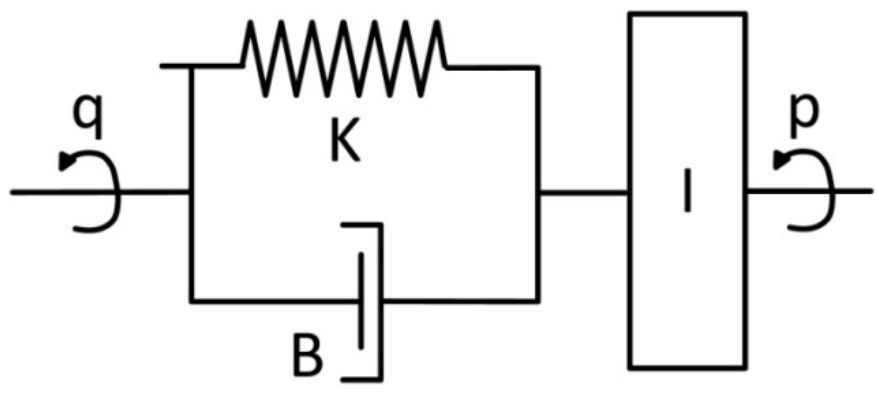

Figure A.5: Mechanical analogon of the semicircular canal (scc), $I=$ endolymph mass inertia, $B=v i s c o u s$ friction, $K=$ cupula restoring force, $q=a n g u l a r$ position of the head, $p=a n g u l a r$ position of the endolymph, $\theta$ =angle of the cupula $(=q-p)$.

The moment of inertia is given by $I \cdot \ddot{p}$, the moment of viscous friction by $B \cdot \dot{\vartheta}$ and the moment of elasticity by $K \cdot \vartheta$, which leads to a second-order differential equation by balancing moments:

$I \cdot \ddot{p}=B \cdot \dot{\vartheta}+K \cdot \vartheta$

with $\ddot{p}$ angular endolymph acceleration, $\dot{q}$ angular head velocity and $\vartheta$ cupula angle. Using $\vartheta=q-p$ and the fact that $I / B\left(\mathrm{~T}_{1} \approx 3 \mathrm{~ms}\right)$ is much smaller than $B / K\left(\mathrm{~T}_{2} \approx 10 \mathrm{~s}\right)$ [Melvill Jones, 1979], the transfer function can in the Laplace domain be written as

$\frac{\vartheta}{\dot{q}}(s)=T_{1} \cdot T_{2} \cdot \frac{s}{\left(T_{1} \cdot s+1\right) \cdot\left(T_{2} \cdot s+1\right)}$

with angular head velocity $\dot{q}$ as input and cupula angle $\vartheta$ as output. The shape of this transfer function is shown in figure A.6.

The scc senses angular acceleration because the endolymph's mass inertia is the driving force, but at physiological frequencies of head movements (about 0.5 to $5 \mathrm{~Hz}$ ) the ssc works as angular velocitymeter [Goldberg and Fernandez, 1971]: the cupula afferent signals are proportional to and in phase with angular head velocity as indicated by the flat response of the transfer function. Additionally, as shown in figure A.7, compensatory eye velocity is proportional to and in anti-phase with head velocity [Huizing et al., 2007]. Cupula deflection (A.7.d) and vestibular nerve firing rate (A.7.e) are proportional and in phase with head velocity (A.7.C). The abducens nerve firing rate (A.7.f) lags the cupula deflection (A.7.d) by 90 degrees, caused by an oculomotor integrator, because the 
lateral rectus muscle contraction needs position-coded information, which is superimposed on the baseline contraction (voluntary eye position). Compensatory eye velocity (A.7.h) is then proportional to and in anti-phase with head velocity (A.7.C).

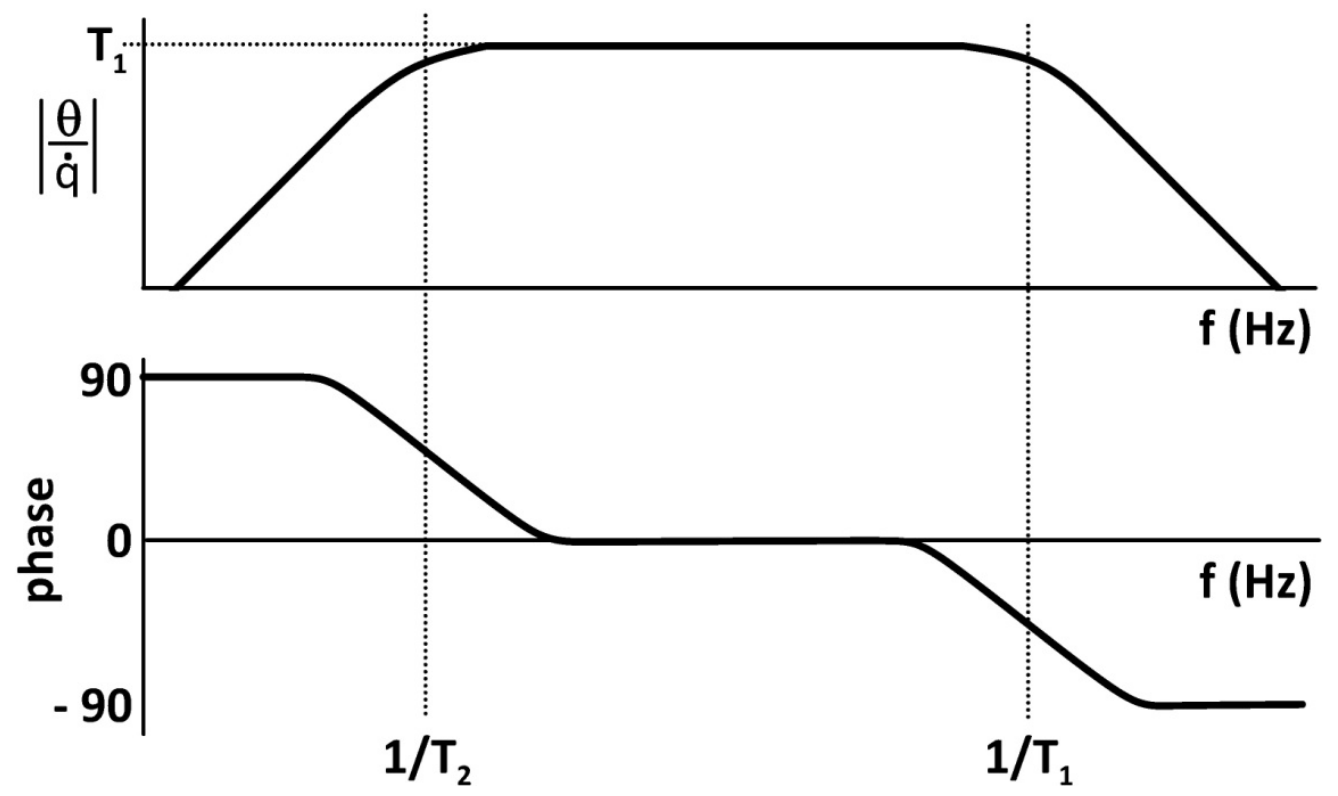

Figure A.6: Bode plot of the frequency response of the transfer function equation 2, representing the dynamic response of the mechanical analogon of the scc. $1 / T_{2}=0.1 \mathrm{~Hz}, 1 / T_{1}=333 \mathrm{~Hz}$ 


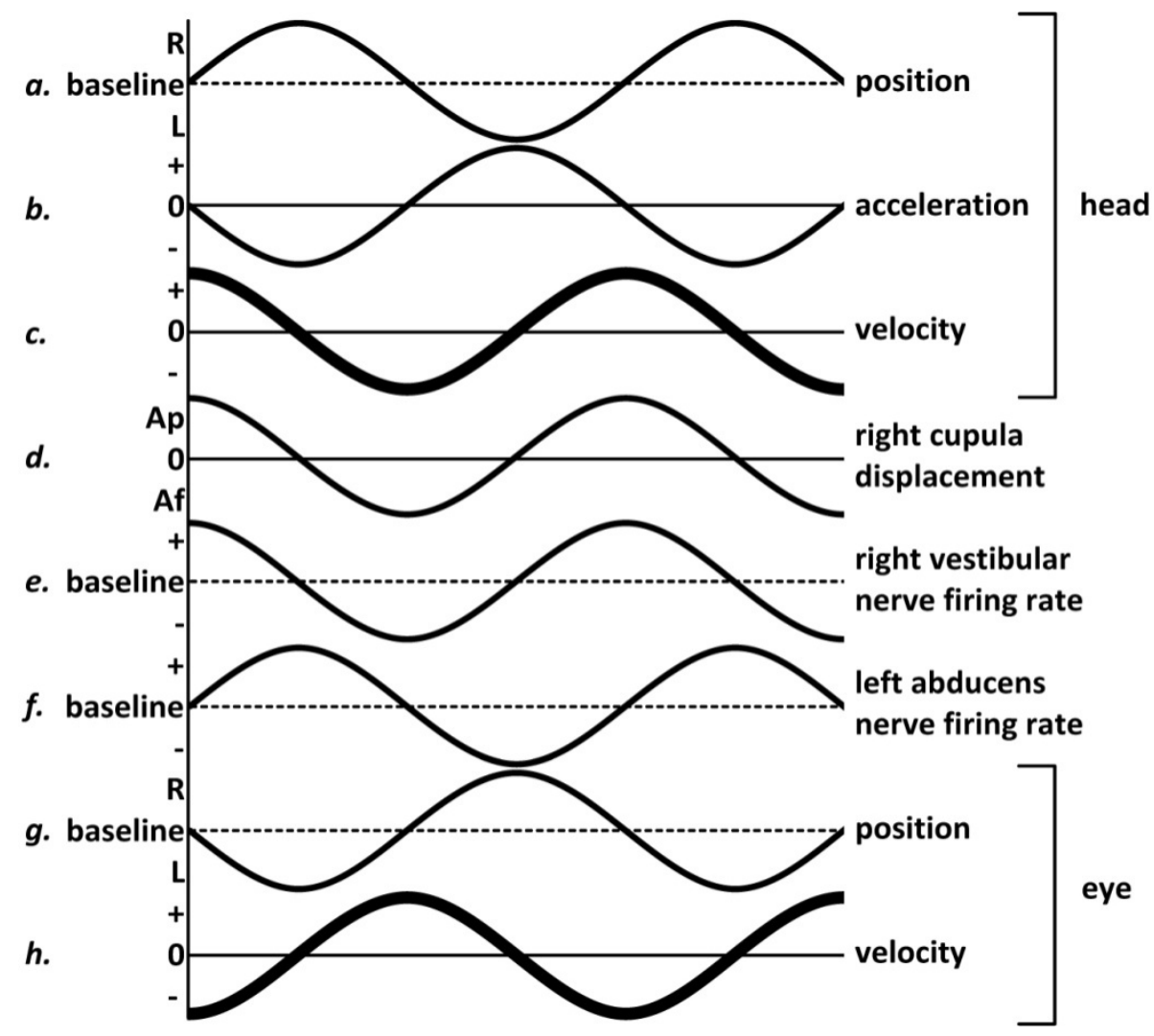

Figure A.7: Mechanism by which a sinusoidal change in head position (a) is converted to an equal and opposite eye position ( $g$ ) at physiological frequencies of head movements, based on [Baloh and Honrubia, 2001]. Cupula deflection (d) and vestibular nerve firing rate (e) are proportional and in phase with head velocity (c). The abducens nerve firing rate (f) lags the cupula deflection (d) by 90 degrees, caused by an oculomotor integrato. Compensatory eye velocity $(h)$ is then proportional to and in anti-phase with head velocity (c).Ap = ampullopetal, $A f=$ ampullofugal

Using two commonly applied paradigms in vestibular clinical investigation, different cupula responses can be explained. Using a constant angular acceleration on a rotational chair, the cupula deflects with the time constant $T_{2}$, as shown in figure A.8.a. A very short angular acceleration (velocity step), illustrated in figure A.8.b, results in a rapid deflection of the cupula with time constant $\mathrm{T}_{1}$ and exponential decay back to zero with time constant $\mathrm{T}_{2}$. 


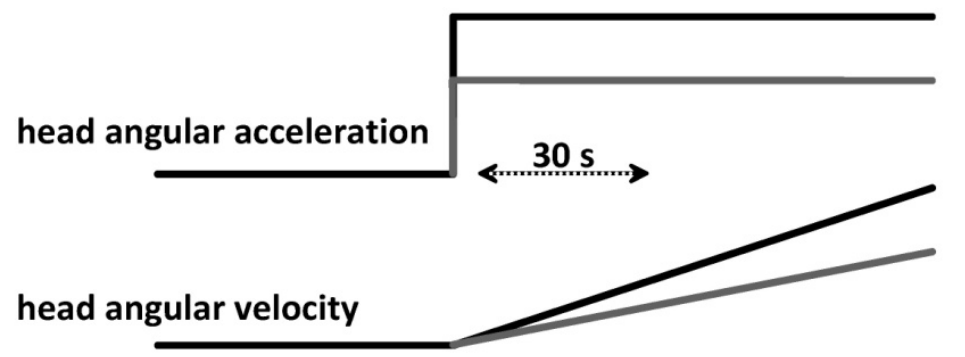

cupula displacement

\section{a. acceleration step}
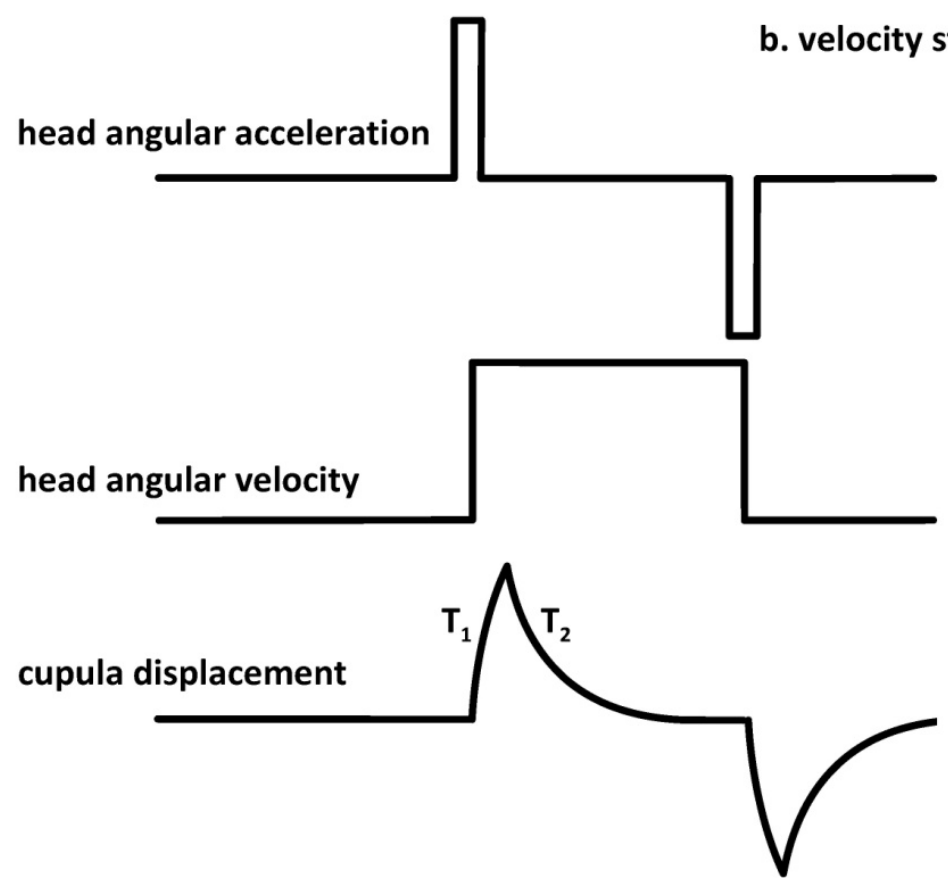

Figure A.8: Schematic cupula responses to a) a step in angular acceleration and b) a velocity step. Grey and black indicate 2 angular acceleration steps with differing amplitudes 


\section{Otolith organs}

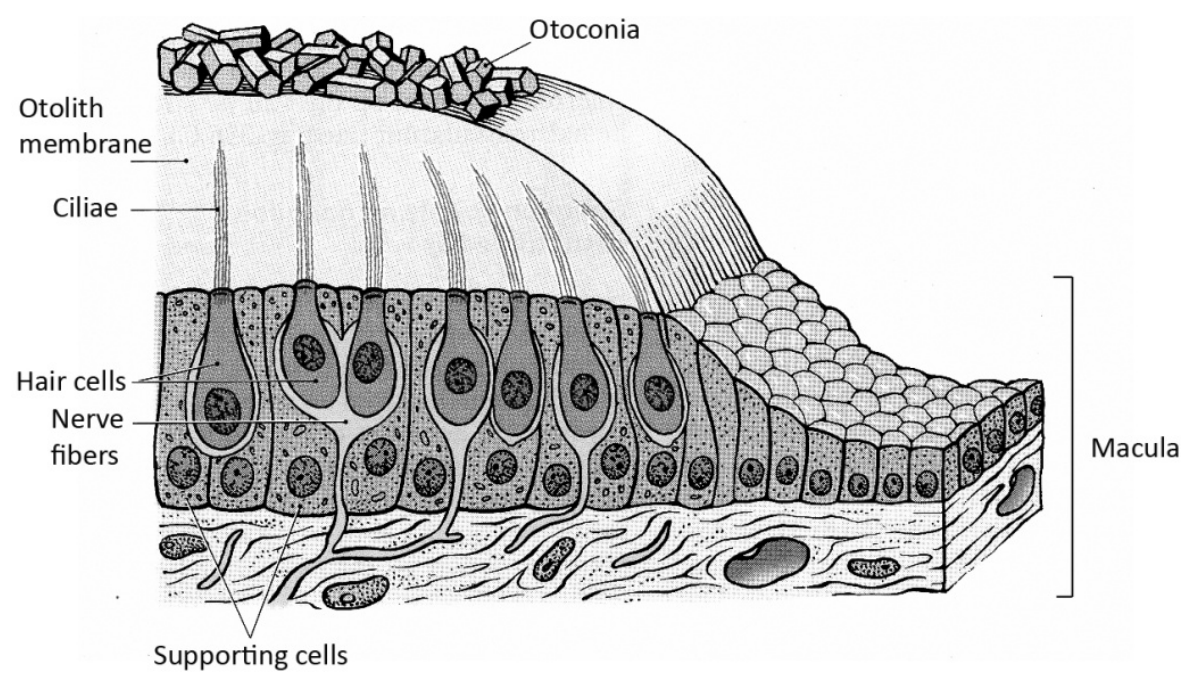

Figure A.9: Schematic representation of the otolith organ, with the macula as sensory epithelium, the otolithic membrane, courtesy of Robby Vanspauwen

A schematic representation of an otolith organ is shown in figure A.9, which is immersed in endolymph fluid [Melvill Jones, 1979]. The macula is the sensory epithelium, with an otolithic membrane which contains the mechanoreceptive hairs and crystalline deposit (otoconia) on top. A linear acceleration of the head causes the otoconia mass to lag behind due to its mass inertia of that otolith organ which is parallel to the plane of motion, thereby bending the otolithic membrane and thus the hair cells, which signal this change to the brain via the $8^{\text {th }}$ nerve. This bending causes depolarization or hyperpolarization of the hair cells depending on the direction of linear acceleration and their polarization on the epithelium. Each macula contains hair cells that are polarized in all directions, in contrast to the scc, resulting in a redundancy of the direction preponderance of the macula in the left and right labyrinth.

During linear head acceleration or head tilt the otoconia mass shifts relative to the macula due to the otoconia mass inertia, causing opposing viscous friction and an elastic force. Therefore the otolith organ can be modeled similar to the scc with a simple mechanical analogon, using inertia (I), viscosity (B) and elasticity (K) as physical quantities (see figure A.10). 


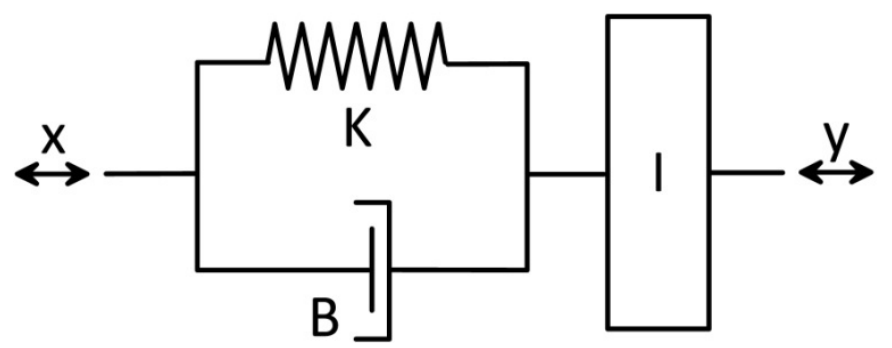

Figure A.10: Mechanical analogon of the otolith organ, I=otoconia mass inertia, $B=$ viscous friction, $K=e l a s t i c$ restoring force, $x=$ position of the head, $y=$ position of the otoconia, $\delta=x-y=$ relative displacement of the otolithic membrane.

The moment of inertia is given by $I \cdot \ddot{y}$, the moment of viscous friction by $B \cdot \dot{\delta}$ and the moment of elasticity by $K \cdot \delta$, which leads to a second-order differential equation similar to the scc. In the case of the otolithic organ however, since the otoconial mass is immersed in endolymph fluid of density $\rho_{e}$, any linear acceleration will generate a buoyancy force acting according to Archimedes' principle in the direction of imposed acceleration and is equal to $\left(\rho_{e} / \rho_{o}\right) \cdot I \cdot \ddot{x}$, with $\rho_{o}$ the density of the otoconial mass. Therefore the second-order differential equation of the otolith organ is:

$\left(1-\frac{\rho_{e}}{\rho_{o}}\right) I \cdot \ddot{x}=I \cdot \ddot{\delta}+B \cdot \dot{\delta}+K \cdot \delta$

with $\ddot{x}$ linear head acceleration, $\ddot{y}$ linear otoconia acceleration, and $\delta$ relative displacement of the otolithic membrane, using $\delta=x-y$ [Melvill Jones, 1979]. The transfer function can in the Laplace domain be written as

$\frac{\delta}{\ddot{x}}(s)=\left(1-\frac{\rho_{e}}{\rho_{o}}\right) \cdot \frac{I}{I \cdot s^{2}+B \cdot s+K}$

with linear head acceleration $\ddot{x}$ as input and relative otolithic membrane displacement $\delta$ as output. The shape of this transfer function is shown in figure A.11, using the fact that $I / B\left(\mathrm{~T}_{1} \approx 0.1 \mathrm{~s}\right)$ is smaller than $B / K\left(\mathrm{~T}_{2} \approx 1 \mathrm{~s}\right)$ [Fernandez and Goldberg, 1976, Melvill Jones, 1979, Bos and Bles, 2002]. 


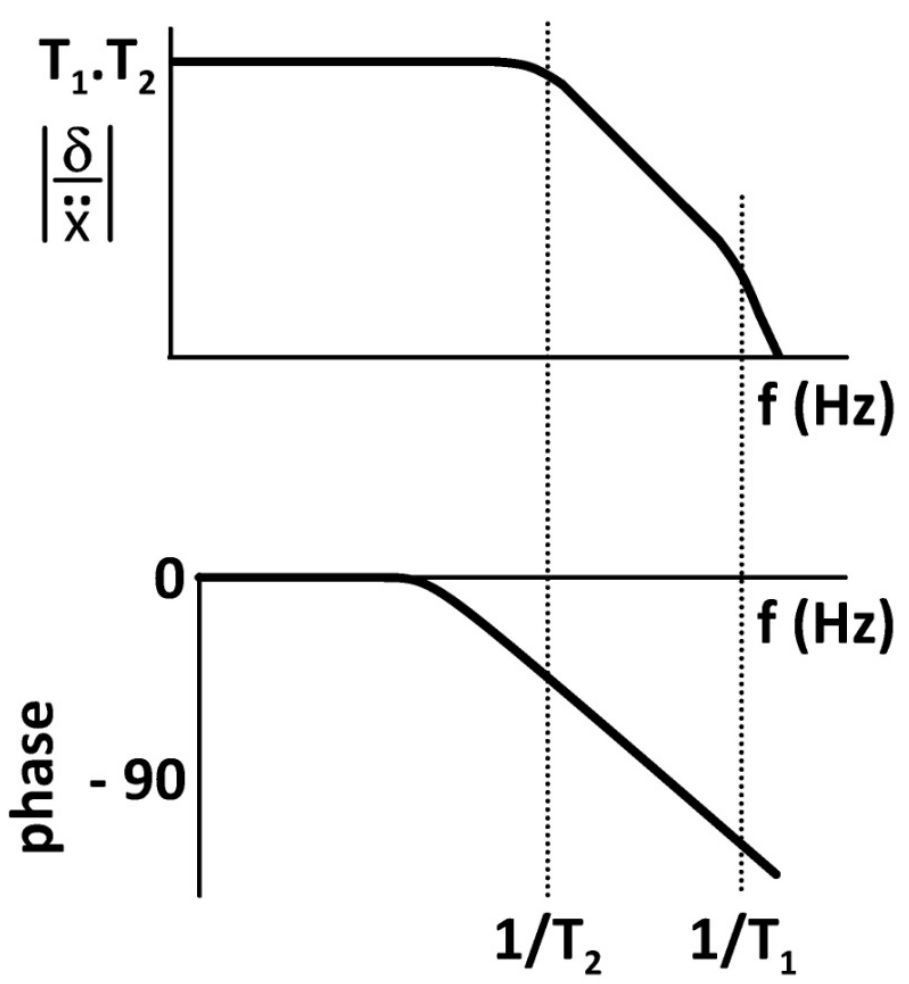

Figure A.11: Bode plot of the frequency response of the transfer function equation 4, representing the dynamic response of the mechanical analogon of the otolith organ. $1 / T_{2}=1 \mathrm{~Hz}, 1 / T_{1}=10 \mathrm{~Hz}$

The otolith organ is sensitive for constant $(0 \mathrm{~Hz})$ and low frequent linear accelerations up to about $1 \mathrm{~Hz}$. Since gravitational acceleration and a corresponding linear acceleration of the system are physically equivalent (Einstein's equivalence principle), the otolith organs cannot distinguish between pure head translations and static head tilts. The relative otolithic membrane displacement $\delta$ in response to a constant linear acceleration is similar to the cupula displacement in response to an angular acceleration as shown in figure A.8.a, but with a time constant $T_{2}$ a factor 10 smaller.

\section{Vestibular organs}

The semicircular canals and otolith organs are both embedded in the vestibular organs and have a complementary functionality because, as explained, the canals sense angular accelerations whereas the otolith system senses linear accelerations of the head. On earth, head movements always occur within the gravitational field and are often 
composed of both rotations and translations. At constant rotational head velocity, the canals are after a while (see figure A.8.b) not stimulated but the otolith system still is due to the centrifugal force. Due to the same force, the otolith system is also stimulated during a change in rotational head velocity, probably supporting a correct interpretation of the output of the canals. A few additional remarks can be made:

- due to the parallel axis theorem [Feynman, 1989], stimulation of an individual canal does not depend on the distance between the axis of rotation and the center of the canal. But the centrifugal component, detected by the otolith system, depends on the location relative to the rotation axis

- a scc measures angular acceleration independently of coincidental linear acceleration [Melvill Jones, 1979] because the cupula and endolymph have the same density. If differences in densities would occur the canal dynamics would be more complex. There would be a dependency on the orientation of both the gravity vector relative to the canal plane and the axis of rotation, as well as on the distance between the axis of rotation and the center of the semicircular canal [Kondrachuk et al., 2008].

This effect can be experienced after too much alcohol intake, resulting in sensations of rotation when lying in bed and can even induce eye movements known as positional alcohol nystagmus [Goldberg, 1966]. This is also the effect experienced in the common vestibular disorder BPPV (Benign Paroxysmal Positional Vertigo). In BPPV, otoconia particles are present in the semicircular canals. These particles make the semicircular canal system sensitive to the orientation of gravity and can adher to the cupula (called cupulolithiasis [Schuknecht, 1962]) or remain freefloating (called canalithiasis [Epley, 1995, Rajguru et al., 2004, Rajguru et al., 2005]).

\section{Vestibular system}

As mentioned before, the vestibular system integrates sensory information from the visual and the somesthic system with information from the vestibular organs, schematically shown in figure A.2. Both the visual and somesthic system can only process relatively slow body movements, and can be modeled with a low pass fiter with a cut-off frequency of about $0.2 \mathrm{~Hz}$. The otolith organs detect low frequent linear accelerations (translations and tilt) up to about $1 \mathrm{~Hz}$, the semicircular canals (scc) detect 
angular velocity above $0.1 \mathrm{~Hz}$. A schematic representation of the gain of these frequency responses is shown in figure $A .12$.

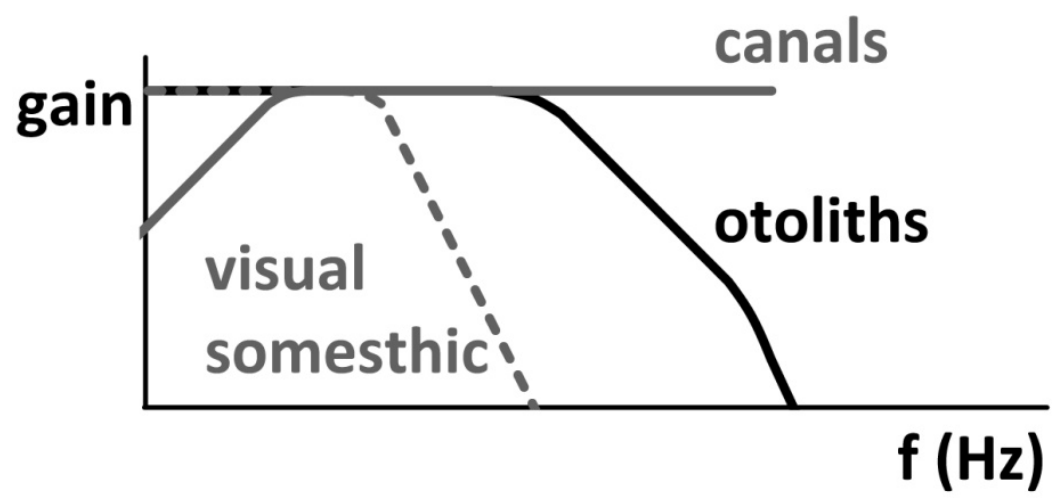

Figure A.12: Schematic representation of the gain of the different frequency responses of vision, somatosensory, and vestibular organs. The visual and somesthic system can be modeled with a low pass filter with $0.2 \mathrm{~Hz}$ cut-off, the otoliths detect low frequent linear accelerations up to about $1 \mathrm{~Hz}$ and the scc detect angular velocity above $0.1 \mathrm{~Hz}$.

The visual and somesthic system support the otolith organs for the detection of constant linear acceleration [Vaugoyeau et al., 2008], whereas the scc support the otolith organs to distinguish true body tilt from translatory movements [Green et al., 2005, Merfeld et al., 2005]. Additionally, the scc drive the angular vestibular ocular reflex and head stabilization during body movements.

As mentioned before (figure A.2), the vestibular system is an adaptive system [Lopez et al., 2007]: walking on the beach and maintaining balance with eyes closed (no visual input and reduced somatosensory information) is possible by relying more on the labyrinthine input. If however different sensory systems give conflicting information, hindering a correct perception of the direction of gravity, motion sickness can be provoked [Bles et al., 1998]. Even worse, when vestibular function is lossed, a permanent impairment remains [Luxon et al., 2003], although as mentioned before and shown in figure A.12 some overlap in functionality of different sensory systems exists. This is especially problematic when for example walking on the beach - reduced somatosensory information and a moving visual horizon -, cycling in the hills - tilted visual scene - or wind surfing - conflicting somatosensory information and a moving visual scene. 


\section{Aging}

Aging affects among others the human's stiffness and hydration properties, and thus can also be expected to affect the physical quantities of both the semicircular canals and otolith organs:

- an increasing stiffness $K$ increases the lower cut-off frequency $(K / B)$ and decreases the gain $(\mathrm{I} / \mathrm{K})$ below this cut-off frequency

- an increasing viscosity $B$ decreases the higher cut-off frequency $(B / I)$ and decreases the gain (I/B) below this cut-off frequency

These effects are schematically shown in figure A.13. The vestibular system as a whole is thus affected as well, deteriorating the distinction between tilt and translation, because the scc optimal range becomes smaller. This is extra unfortunate because at higher age body movements become slower due to stiffer body mechanics.

semicircular canals

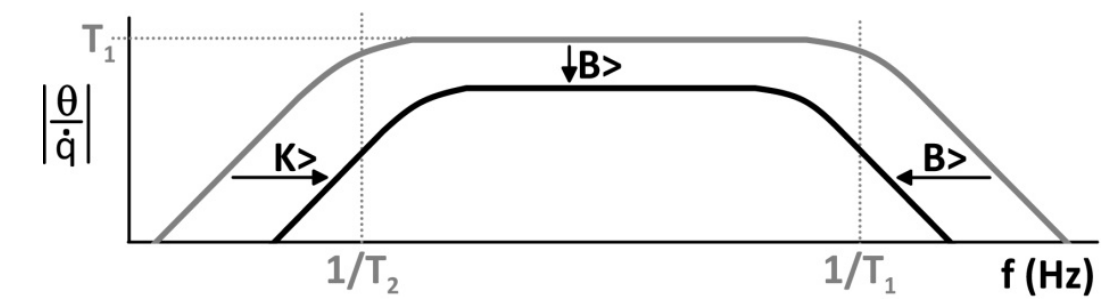

otolith organs

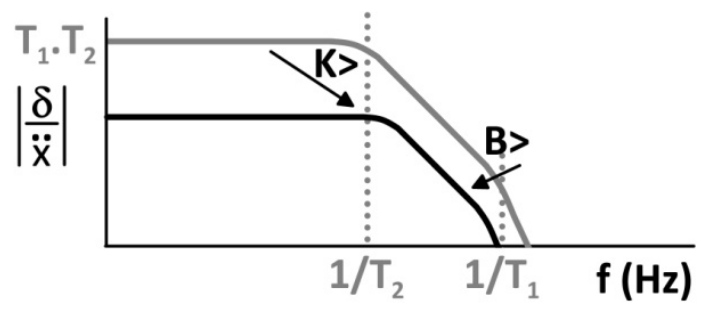

Figure A.13: Effect of aging (increasing stiffness $K$ and increasing viscosity $B$, indicated by the arrows) on the gain of the frequency responses of the semicircular canals (equation 2) and the otolith organs (equation 4) 


\section{Aim and outline of this thesis}

The aim of this research was to evaluate and improve existing and to develop advanced diagnostic and therapeutic possibilities for vestibular medicine: first of all to improve vestibular diagnostics and therapy in general and therefore to be able in the (near) future to select vestibular patients for specific rehabilitation programs and therapeutic options.

This thesis is built around the three major properties of the vestibular system. Part B focuses on an advanced therapeutic method to improve postural control of vestibular patients; the ambulatory vibrotactile biofeedback (AVBF) system. In part $\mathrm{C}$ perception of rotations, translations and tilts is discussed. Part $D$ focuses on improvement of an advanced diagnostic method to visualize and quantify eye movements, the infrared video eye tracker. As a basis, this part introduces a theoretical background. 


\section{References}

Baloh, R. W. \& Honrubia, V. (2001) Clinical Neurophysiology of the Vestibular System. 3 ed.

Bles W., Bos J. E., de Graaf B., Groen E. \& Wertheim A.H. (1998) Motion sickness: only one provocative conflict? Brain Res Bull, 47, 481-7

Bos, J. E. \& Bles, W. (2002) Theoretical considerations on canal-otolith interaction and an observer model. Biol Cybern, 86, 191-207.

Bringoux, L., Nougier, V., Barraud, P. A., Marin, L. \& Raphel, C. (2003) Contribution of somesthetic information to the perception of body orientation in the pitch dimension. $Q J$ Exp Psychol A, 56, 909-23.

Epley J. M. (1995) Positional vertigo related to semicircular canalithiasis. Otolaryngol Head Neck Surg, 112, 154-61

Fernandez, C. \& Goldberg, J. M. (1976) Physiology of peripheral neurons innervating otolith organs of the squirrel monkey. II. Directional selectivity and force-response relations. $J$ Neurophysiol, 39, 985-95.

Feynman, R. P. (1989) The Feynman lectures on Physics.

Goldberg, J. M. \& Fernandez, C. (1971) Physiology of peripheral neurons innervating semicircular canals of the squirrel monkey. I. Resting discharge and response to constant angular accelerations. J Neurophysiol, 34, 635-60.

Goldberg, L. (1966) Behavioral and Physiological Effects of Alcohol on Man. Psychosomatic Medicine, 28, 570-595.

Green, A. M., Shaikh, A. G. \& Angelaki, D. E. (2005) Sensory vestibular contributions to constructing internal models of self-motion. J Neural Eng, 2, S164-79.

Huizing, E. H., Snow, G. B. \& Graamans, K. (2007) Keel-neus-oorheelkunde en hoofd-halschirurgie. 3 ed.

Kingma, H. (2006) Function tests of the otolith or statolith system. Curr Opin Neurol, 19, 21-5.

Kondrachuk, A. V., Sirenko, S. P. \& Boyle, R. (2008) Effect of difference of cupula and endolymph densities on the dynamics of semicircular canal. J Vestib Res, 18, 69-88.

Lopez, C., Lacour, M., Ahmadi, A. E., Magnan, J. \& Borel, L. (2007) Changes of visual vertical perception: a long-term sign of unilateral and bilateral vestibular loss. Neuropsychologia, 45, 2025-37.

Luxon, L. M., Martini, A., Furman, J. M. \& Stephens, D. D. G. (2003) Audiological Medicine.

Mahboobin, A., Loughlin, P., Atkeson, C. \& Redfern, M. (2009) A mechanism for sensory reweighting in postural control. Med Biol Eng Comput.

Melvill Jones, G. (1979) Biophysics of the Peripheral End Organs. IN WILSON, V. J. \& MELVILLJONES, G. (Eds.) Mammalian vestibular physiology. New York, Plenum Press.

Merfeld, D. M., Park, S., Gianna-Poulin, C., Black, F. O. \& Wood, S. (2005) Vestibular perception and action employ qualitatively different mechanisms. I. Frequency response of VOR and perceptual responses during Translation and Tilt. J Neurophysiol, 94, 186-98.

Merfeld, D.M., Priesol A., Lee, D., Lewis, R.F. (2010) Potential solutions to several vestibular challenges facing clinicians. J Vest Res, 20, 71-77

Rader, A. A., Oman, C. M. \& Merfeld, D. M. (2009) Motion perception during variable-radius swing motion in darkness. J Neurophysiol, 102, 2232-44. 
Rajguru, S. M., Ifediba, M. A. \& Rabbitt, R. D. (2004) Three-dimensional biomechanical model of benign paroxysmal positional vertigo. Ann Biomed Eng, 32, 831-46.

Rajguru, S. M., Ifediba, M. A. \& Rabbitt, R. D. (2005) Biomechanics of horizontal canal benign paroxysmal positional vertigo. J Vestib Res, 15, 203-14.

Schuknecht, H. F. (1962) Positional vertigo: clinical and experimental observations. Trans Am Acad Opthalmol Otol, 66, 319-331.

Stolbkov, Y. K. \& Orlov, I. V. (2009) Artificial vestibular feedback in conditions of a modified body scheme. Neurosci Behav Physiol, 39, 173-81.

Vaugoyeau, M., Viel, S., Amblard, B., Azulay, J. P. \& Assaiante, C. (2008) Proprioceptive contribution of postural control as assessed from very slow oscillations of the support in healthy humans. Gait Posture, 27, 294-302. 
Part A 


\section{Part B}

CONTROL OF POSTURE
In this part, the effects of the ambulatory vibrotactile biofeedback (AVBF) system on orientation, stance and gait were explored. Healthy subjects, while seated on a stool on a tilting platform in a light-tight room, had to reorient the platform back to absolute horizontal using a velocity controlled joystick. It was demonstrated that the AVBF system has the ability to change earth vertical orientation.

In patients with severe bilateral vestibular hyporeflexia observational gait analysis was used to score individual balance during gait: significant improvements were shown in our patients using the AVBF system. Only 2 patients showed a significant individual gait improvement with the AVBF system,

but in the majority of our patients it increased confidence and a feeling of balance.

In another group of patients with severe bilateral vestibular losses a placebo-controlled study was performed to explore the effect of the AVBF system on body sway in stance. In $60 \%$ of the patients no significant change in body sway was observed. In 40 $\%$ of the patients body sway decreased significantly using biofeedback, but this improvement was only observed where an improvement was present with placebo biofeedback as well. The improvement was, at least partially, caused by other effects than biofeedback, like training, increased self confidence or alertness.

This part indicates the feasibility of vibrotactile biofeedback for vestibular rehabilitation and to improve balance in stance and during gait, although other effects than biofeedback play a significant role. 
This part would not have been possible without the work from

my graduate students Rianne Pas, Vera Nijssen, Marloes

Damen and Herman Assink, the help from my trainees Christine

Nabuurs, Josine Stammen and Christel van Loo, the technical

support from Jos Aarts, Vincent Kerkhofs and Erik Brands and

the technicians Ellen Rikers, Sophie Paredis and Marie-Cécile

Gerards from the vestibular department.

\section{Based on}

Janssen $M$, Stokroos $R$, Aarts J, van Lummel $R$ and Kingma $H$. Salient and placebo vibrotactile feedback are equally effective in reducing sway in bilateral vestibular loss patients. Gait Posture 2010; 31: 213-7

and

Janssen $M$, Pas $R$, Aarts J, Janssen-Potten $Y$, Vles $H$, Nabuurs $C$, van Lummel $R$, Stokroos $R$ and Kingma $H$. Clinical observational gait analysis to evaluate improvement of balance during gait with vibrotactile biofeedback. Phys Res Int 2011 
The vestibular labyrinths play a key role in posture and balance, retinal image stabilization and spatial orientation that all are affected in case of substantial vestibular deficits, and lead to a major handicap (oscillopsia, postural imbalance) in patients with a bilateral vestibular areflexia. Central compensation in combination with sensory substitution might reduce impairment, but is unlikely to restore full functionality. A potent aid for these patients might be an artificial labyrinth to restore the feedback of linear and angular accelerations of the head or body to the brain. Several researchers are currently engaged with the development of such a device. Some of them try to improve performance in posture and balance in humans using (non-implantable) sensory substitution - galvanic vestibular stimulation [Orlov et al., 2008, Scinicariello et al., 2001], auditory feedback [Hegeman et al., 2005], visual feedback [Hirvonen et al., 1997], electrotactile stimulation of the tongue [Tyler et al., 2003] or vibrotactile feedback to the trunk [Lewis et al., 2002], while others try to restore image stabilization by implanting electrodes to restore the input to the brain in animals [Lewis et al., 2002, Della Santina et al., 2005] or in humans [Wall et al., 2007].

Implantation of electrodes may be not the first option for patients as not everyone may want or need an implantable prosthesis [Wall et al., 2002], but even more because of the invasive aspect and the possible severe vegative symptoms [Yates and Bronstein, 2005]. Sensory substitution prostheses for vestibular biofeedback can be developed in a fairly short time and reduction of sway (in both anteroposterior and mediolateral direction) is possible using biofeedback based on information about an individual's posture. Several options for sensory substitution are available.

Galvanic vestibular stimulation (GVS) has been suggested to improve balance control [Orlov et al., 2008, Scinicariello et al., 2001] in case of labyrinthine deficits. However, habituation to galvanic stimuli is a major issue that reduces and changes the impact of GVS relatively fast [Balter et al., 2004]. Also, many patients investigated in our clinic with GVS, report substantial nausea and pain in the skin under the electrodes in case of prolonged galvanic stimulation.

Auditory feedback [Basta et al., 2008, Hegeman et al., 2005, Ernst et al., 2007, Dozza et al., 2004, Dozza et al., 2005a, Dozza et al., 2005b] and visual feedback [Hirvonen et al., 1997] could be used as well, although 1) visual and auditory inputs are already extensively used by patients with vestibular deficits [Alahakone and Senanayake, 2010], 
2) these patients tend to rely even more on the primary function of the visual and auditory systems in challenging situations and 3 ) communication might be hindered.

Electrotactile [Tyler et al., 2003, Danilov, 2004, Danilov et al., 2006, Danilov et al., 2007] and vibrotactile [Kentala et al., 2003, Dozza et al., 2007, Goebel et al., 2009, Wall et al., 2001, Asseman et al., 2007] feedback systems also seem to be suitable for vestibular substitution. Electrotactile feedback through the tongue is an elegant method to improve posture and balance, as it is a fully head-based system and because a learningeffect of several hours after removal of the prothesis has been shown [Danilov, 2004]. Although, it has disadvantages in daily life, both esthetic and practical (talking and eating).

Vibrotactile feedback through the trunk, stimulating various cutaneous receptors [Mahns et al., 2006, Jones and Sarter, 2008], is a very intuitive approach and has been used in military applications for navigation in combat and as support orientation for blind people [Ram and Sharf, 1998], in industrial telemanipulators [Dennerlein et al., 1997] and in virtual environments [Okamura et al., 1998]. Therefore an ambulatory vibrotactile biofeedback (AVBF) system to reduce body sway and increase postural stability for patients with vestibular dysfunction was developed, based on the approach used by Wall III and colleagues [Wall et al., 2001].

\section{Ambulatory vibrotactile biofeedback (AVBF) system}

The AVBF system, schematically shown in figure B.1, consists of four major components:

1. a DynaPort MiniMod (McRoberts, $5.5 \mathrm{mG}(1 \mathrm{mG} \approx 1 \mathrm{~cm} / \mathrm{s} 2)$ or $0.30^{\circ}$ resolution at a sample frequency of $50 \mathrm{~Hz}$ ) virtual zero drift sensor, small and light weight (64×62×13 mm, 55 gram), containing 3 orthogonal linear (piezo)capacitive accelerometers, which can be mounted on the subject's head or high on the trunk

2. an elastic belt with 12 equally distributed actuators (ZUB.NO32.VIB, eccentric vibra-motors like applied in Nokia 3210 at $300 \mathrm{~Hz}$ and an amplitude of $0.5 \mathrm{~mm}$ [Jones and Sarter, 2008]) around the waist mounted with a Velcro fastener

3. an ATMEGA128 (Atmel) processor to translate sensor output into activation of correct actuators with a delay of $<1 \mathrm{~ms}$

4. a LiPo battery pack (11.1V, $3270 \mathrm{mAh})$ to supply power to all components, thus making the AVBF system an ambulatory, comfortable and simple system 
The battery pack and processor unit dimensions are $12 \times 7 \times 3 \mathrm{~cm}$, weighing 330 gram and 240 gram respectively. The battery can power the processor, actuators and sensor for 72 hours continuously, and can be recharged within 8 hours, making sure that subjects can use the AVBF system for several days and recharge it overnight even without the explicit need of a spare battery.
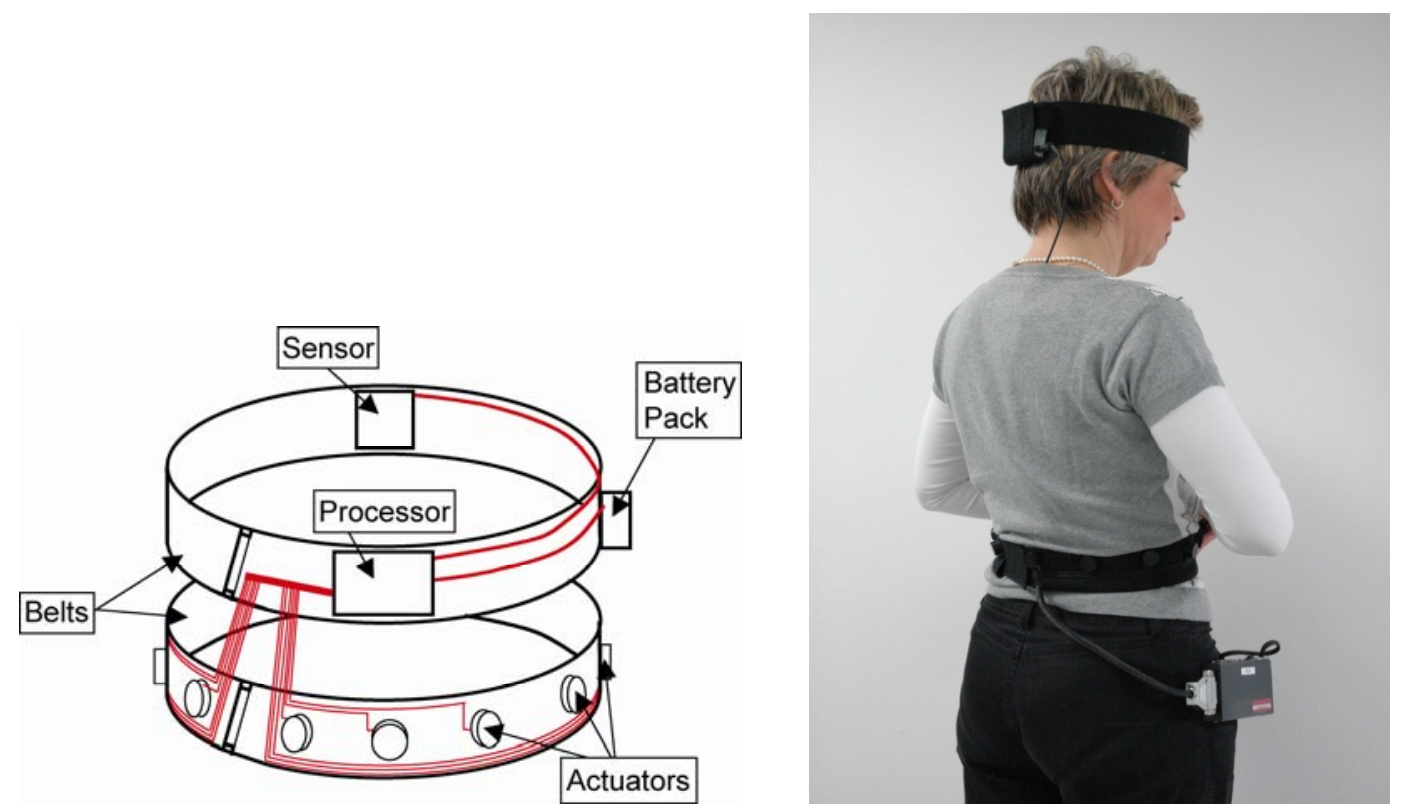

Figure B.1: Schematic overview and a picture of the ambulatory vibrotactile biofeedback (AVBF) system. Details are described in the text. The processor and battery pack can be detached from the upper belt and for exampled fastened on the trousers, having the upper belt only containing the sensor. The sensor can be mounted on the subject's head or high on the trunk.

A subject wearing the AVBF system can set a vertical reference vector at any desired moment, simply by pressing a button on the processor unit. Setting this reference vector is necessary for the AVBF system to know its sensor orientation. Subsequently the processor calculates the vector difference between the reference vector and the current sensor orientation. This vector difference is the subject's tilt angle (size/angle/magnitude and direction) and is translated into the activation of specific actuators. The subject's tilt magnitude and tilt direction are translated into the activation of specific actuators. 
In chapter 1 it will be demonstrated that the AVBF system changes earth vertical orientation in subjects with normal vestibular function, which demonstrates the effectiveness of our AVBF system. The individual use of the AVBF system to increase postural stability in patients with severe bilateral vestibular losses is examined in chapter 2 during gait and in chapter 3 in stance. In chapter 3 , the effect of the AVBF system on stance was evaluated in a placebo controlled way. 


\section{CHAPTER 1: EFFECTIVENESS OF VIBROTACTILE BIOFEEDBACK IN HEALTHY SUBJECTS ON A TILTING PLATFORM}

The goal of this study was to demonstrate that the AVBF system changes the ability of subjects with normal vestibular function to remain oriented to earth vertical during postural perturbations [Peterka et al., 2006]. Body orientation was perturbed by pseudorandom platform tilts upon which the subjects were seated. A seated instead of standing condition was chosen because potential useful ankle and hip angles, basic components for inverted pendulum models of body dynamics to align a body to gravity [Hemami et al., 1978, Kuo, 1995], can be avoided.

\subsection{Materials and methods}

\section{AVBF system activation scheme}

In this study, one actuator is activated in the direction of a subject's body tilt if it exceeds a tilt magnitude of $2^{\circ}$, based on our observations and knowledge that the limit of stability in healthy subjects is about $6^{\circ}$ [Nashner et al., 1989] and the typical sway angle in healthy subjects is about $0.5^{\circ}$ [Horlings et al., 2008]. In this way the AVBF system can code body tilt in any direction and the actuator which is activated above $2^{\circ}$ of tilt indicates the tilt direction. When the subject correctly responds to this actuator, it will be deactivated when the tilt magnitude drops below $1.5^{\circ}$. The range between $1.5^{\circ}$ and $2^{\circ}$ was chosen to avoid abrupt changes in on and off switching of a specific actuator (in other words, hysteresis is induced). Thus, the dead zone has a size of $2^{\circ}$. Figure B.2 shows a schematic representation of the translation of body tilt angle to actuator activation. 


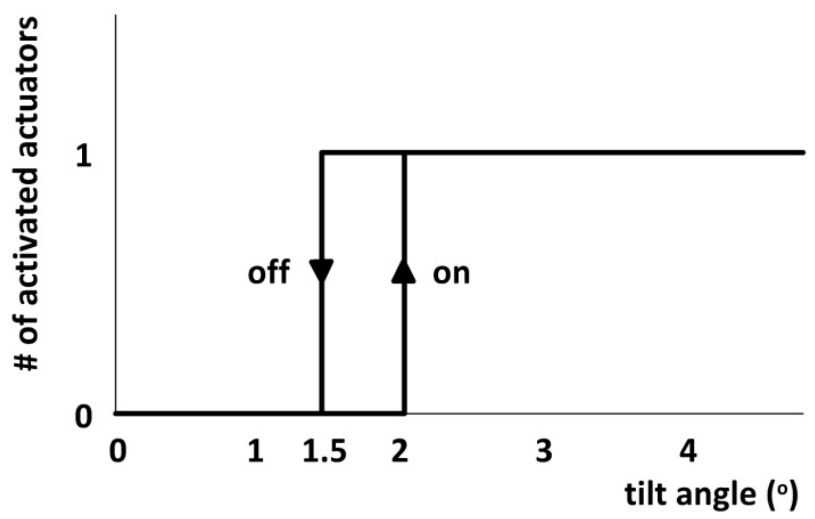

Figure B.2: Activation scheme of the actuator belt worn around the waist. One actuator is activated in the direction of a subject's body tilt when the AVBF system signals a tilt angle of $2^{\circ}$ or more and is deactivated for tilt angles below $1.5^{\circ}$.

\section{Tilt platform}

Whole body tilts while seated were generated by a tilting platform (Maastricht Instruments BV) in roll (left-right) and pitch (nose up-down) direction randomly. The platform was controlled by an embedded computer, which tilted the platform to preprogrammed tilt orientations with a $0.01^{\circ}$ resolution and $1 \mathrm{kHz}$ sample rate with a tilt velocity of $0.1 \%$ (rise time of about $0.1 \mathrm{~s}$ ).

Subjects were seated [Bringoux et al., 2002, Bisdorff et al., 1996] on a stool positioned on the platform without restraints, with their feet on a foam footrest (to avoid potential tactile cues from the platform), eyes closed and masked, hands holding a joystick, and headphones to mask auditory cues for spatial orientation and to communicate with the operator. To further minimize possible visual cues, the platform was tilted in a light-tight room.

\section{Profile}

A platform tilt velocity of $0.1 \%$ (sub canal threshold) was used to reach 15 random platform tilt orientations, with maximum $6^{\circ}$ roll tilts and $4{ }^{\circ}$ pitch tilts. Then the subjects reoriented the platform back to absolute horizontal using a velocity controlled joystick, inducing platform tilts in the full horizontal plane with a maximum velocity of $5 \% \mathrm{~s}$. After the subjects' indication that the platform was horizontal again, the operator pushed a 
button to move the platform to the next orientation. Total profile time was on average $12 \mathrm{~min}$.

\section{Healthy subjects}

Nine subjects ( 6 males, 3 females, mean age 28 ( 23 - 36) years) without any known history or evidence of any ophthalmologic, neurologic or vestibular disorder participated in this study. All subjects participated on a voluntary basis after giving their informed consent.

\section{Procedure}

Each subject had 1 minute to familiarize with the AVBF system with biofeedback on the waist and sensor on the trunk. They quickly learned how to use the system and to experience the relation between trunk orientation and actuators. Then the AVBF system was switched off, they were seated on the stool on the platform with their feet on the foam footrest and the joystick was given to them.

They were instructed, with the AVBF system switched off, to orient the platform to absolute horizontal using the joystick. Two random platform tilts were given to familiarize subjects with the joystick functionality, before closing and masking the eyes and mounting the headphones. They were then instructed that the platform would tilt to random positions and that they needed to orient the platform back to absolute horizontal using the joystick, after the platform had reached its position. They were instructed to verbally indicate that the platform was horizontal and the current platform orientation was saved. They were also instructed not to communicate with the operator, except when they needed to indicate horizontal platform orientation or when they wanted to abort the study (which never happened). Thereafter the platform tilted to the next random orientation while the AVBF system remained switched off until a total of 15 platform reorientations back to horizontal had been performed.

Then the AVBF system was switched on and the AVBF's reference vector was set, while the subjects' eyes were still closed and masked and the headphones were still mounted. They were instructed that the platform would tilt to random positions and that they needed to orient the platform back to absolute horizontal using the joystick, after the platform had reached its position. They were also instructed to verbally indicate that the platform was horizontal and the current platform orientation was saved. And they were instructed not to communicate with the operator, except when they needed to indicate 
horizontal platform orientation or when they wanted to abort the study (which never happened).

Table B.1: Individual mean and standard deviation of the 15 saved platform orientations in both roll and pitch direction. Within each subject (\#) the Mann-Whitney $U$ test was used to determine significant different platform orientations between both AVBF settings (on and off). The green cells indicate the significant $(p<0.01)$ individual orientation differences and the setting in which the platform was more horizontaol. Three different AVBF biofeedback strategies were used when the AVBF system was switched on: oriented the platform in such a way that the AVBF system stopped vibrating (within the AVBF dead zone) and then 1) indicated that the platform was horizontal, 2) used all other available bodily sensations to fine tune the platform orientation and then indicated that the platform was horizontal, or 3) completely ignored the vibrotactile biofeedback during the platform tilts.

\begin{tabular}{|c|c|c|c|c|c|c|c|c|c|c|}
\hline \multirow[t]{2}{*}{$\#$} & \multirow[t]{2}{*}{$\mathrm{m} / \mathrm{f}$} & \multirow[t]{2}{*}{ Age } & \multirow[t]{2}{*}{ AVBF } & \multicolumn{3}{|l|}{ Roll } & \multicolumn{3}{|l|}{ Pitch } & \multirow{2}{*}{$\begin{array}{l}\text { Biofeedback } \\
\text { usage }\end{array}$} \\
\hline & & & & mean & $\Sigma$ & $\begin{array}{l}\text { More } \\
\text { horizontal }\end{array}$ & mean & $\sigma$ & $\begin{array}{l}\text { More } \\
\text { horizontal }\end{array}$ & \\
\hline \multirow{2}{*}{1} & \multirow{2}{*}{$M$} & \multirow{2}{*}{32} & off & -0.3 & 0.37 & \multirow{2}{*}{ On } & -0.5 & 0.6 & \multirow{2}{*}{ on } & \multirow{2}{*}{1} \\
\hline & & & on & 0.15 & \begin{tabular}{|l|l}
0.44 \\
\end{tabular} & & -4 & 1.64 & & \\
\hline \multirow{2}{*}{2} & \multirow{2}{*}{$\mathrm{F}$} & \multirow{2}{*}{23} & off & -1.5 & 0.57 & \multirow{2}{*}{ On } & 0.1 & 0.65 & \multirow{2}{*}{ on } & \multirow{2}{*}{2} \\
\hline & & & on & -0.6 & 0.6 & & -0.6 & 0.53 & & \\
\hline \multirow{2}{*}{3} & \multirow{2}{*}{$M$} & \multirow{2}{*}{24} & off & -0.3 & 0.48 & \multirow{2}{*}{ On } & 0.5 & 0.7 & \multirow{2}{*}{ on } & \multirow{2}{*}{1} \\
\hline & & & on & -0.2 & 0.41 & & 3.59 & 0.49 & & \\
\hline \multirow{2}{*}{4} & \multirow{2}{*}{$M$} & \multirow{2}{*}{31} & off & 0.89 & 0.54 & \multirow{2}{*}{ On } & 0.24 & 0.27 & \multirow{2}{*}{ off } & \multirow{2}{*}{2} \\
\hline & & & on & 0.62 & 0.61 & & 0.1 & 0.83 & & \\
\hline \multirow{2}{*}{5} & \multirow{2}{*}{$M$} & \multirow{2}{*}{28} & off & -0.3 & 0.6 & fff & 1.05 & 0.84 & & 1 \\
\hline & & & on & 0.59 & 0.62 & UTा & -1.6 & 0.72 & on & 1 \\
\hline & & & off & \begin{tabular}{|c|}
-0.1 \\
\end{tabular} & \begin{tabular}{|l|}
0.41 \\
\end{tabular} & off & $\begin{array}{l}-0.9 \\
\end{array}$ & 0.44 & $0 n$ & ? \\
\hline 0 & $\mathrm{IVI}$ & 30 & on & -0.4 & 0.7 & $0 \pi$ & -0.2 & 0.72 & on & 2 \\
\hline 7 & $M$ & 28 & off & -0.6 & 1.09 & fff & -0.5 & 0.77 & on & 1 \\
\hline$f$ & $\mathrm{IVI}$ & $\angle 8$ & on & 1.6 & \begin{tabular}{|l|}
1.15 \\
\end{tabular} & UIT & \begin{tabular}{l|l}
-0.1 \\
\end{tabular} & 0.88 & on & 1 \\
\hline 8 & & & off & 0.13 & 0.48 & Off & -0.3 & 0.56 & & 1 \\
\hline 8 & $r$ & 25 & $\begin{array}{l}\text { on } \\
\end{array}$ & 1.78 & \begin{tabular}{|l}
1.71 \\
\end{tabular} & UTI & 0.06 & 0.68 & on & 1 \\
\hline & & & off & -0.2 & 0.82 & & -0.1 & 1.09 & ff & ? \\
\hline 9 & $r$ & 30 & $\begin{array}{l}\text { on } \\
\end{array}$ & 0.05 & \begin{tabular}{|l|l}
0.85 \\
\end{tabular} & on & 0.45 & 0.87 & $\mathrm{Otf}$ & 3 \\
\hline
\end{tabular}


Thereafter the platform tilted to the next random orientation while the AVBF system remained switched on until a total of 15 platform reorientations back to horizontal had been performed.

As we noticed in a pilot study that, despite the strict description, naïve subjects interpreted the instructions in different ways. Therefore all subjects were asked at the end to briefly describe how if and how they had used the vibrotactile biofeedback to reorient the platform back to horizontal.

\section{Data analysis}

For each subject and AVBF setting (off or on) mean and standard deviation of the 15 saved platform orientations were calculated in both roll and pitch direction. Within each subject the Mann-Whitney $U$ test (non-parametric two-independent-samples test) was used to determine possible significant different platform orientations between both AVBF settings. To determine possible significant differences in mean and standard deviation between both AVBF settings on a group level, Wilcoxon signed rank test (nonparametric two-related-samples test) was used. The level of significance in all tests applied was $p<0.05$.

\subsection{Results}

The subjects' results are shown in table B.1. After each experiment all subjects described how they managed to fulfill the task. One subject (\# 9) indicated that she had completely ignored the vibrotactile biofeedback during the platform tilts when the AVBF system was switched on. The other 8 subjects indicated they used the vibrotactile biofeedback to orient the platform, using 2 different strategies:

1. 5 subjects oriented the platform in such a way that the AVBF system stopped vibrating (within the AVBF dead zone) and then indicated that the platform was horizontal, although they sometimes noticed that the platform was not horizontal at all. (AVBF strategy 1 in table B.1)

2. 3 subjects oriented the platform in such a way that the AVBF system stopped vibrating and then used all other available bodily sensations to fine tune the platform orientation. They then indicated that the platform was horizontal, although they sometimes noticed that the platform was not horizontal at all. (AVBF strategy 2 in table B.1) 
These 8 subjects were then again told that, even with the AVBF system switched on, the instruction had been to orient the platform back to absolute horizontal using the joystick. They all indicated that they had interpreted this instruction as orient the platform as horizontal as possible without getting any vibration in these orientations.

Seven subjects oriented the platform significantly $(p<0.01)$ different between both AVBF settings (switched off and on). The mean and standard deviation were not significantly different between AVBF system switched off and on (mean: $p=0.07$ and 0.95 and standard deviation: $p=0.07$ and 0.33 , for roll and pitch respectively).

\subsection{Discussion and conclusion}

Based on the subjective responses of the 9 healthy subjects investigated, the subjects were not always able to keep their body orientation perpendicular to the platform surface. They sometimes noticed that the platform was tilted, although the AVBF system was not giving vibrotactile biofeedback and the AVBF sensor was thus aligned with the initial reference orientation. In other words, the body orientation had changed during the procedure with the AVBF system switched on, and they sometimes adjusted the platform orientation in such a way that the AVBF system did not give any vibration biofeedback (dead zone); although they still noticed that the platform was not horizontal. This demonstrates the effectiveness of our AVBF system and its ability to change earth vertical orientation of subjects with normal vestibular function. 


\section{CHAPTER 2: CLINICAL OBSERVATIONAL GAIT ANALYSIS TO EVALUATE IMPROVEMENT OF BALANCE DURING GAIT WITH VIBROTACTILE BIOFEEDBACK}

In this study we focus on the use of the AVBF system to increase postural stability during gait in patients with severe bilateral vestibular losses. To our knowledge only 2 studies on the effect of biofeedback on gait have been published [Hegeman et al., 2005, Dozza et al., 2007]. Hegeman et al. measured trunk sway during different gait tasks using two angular velocity transducers, while Dozza et al. measured motion kinematics using a Motion Analysis system. We assess gait performance, of patients with various pathologies (including spasticity and severe vestibular hyporeflexia), in our clinical movement laboratory with a Sybar system [Harlaar et al., 2000] to register and interpret gait and to evaluate treatment based on observation.

\subsection{Materials and methods}

\section{AVBF system activation scheme}

Figure B.3 shows a schematic representation of the translation of body tilt angle to actuator activation used in this study. The number of activated actuators is based on the sensor resolution and chosen to avoid sensory adaptation due to continuous vibrotactile stimulation. The activation scheme is based on our observations and knowledge that the limit of stability in healthy subjects is about $6^{\circ}$ [Nashner et al., 1989] and the typical sway angle in healthy subjects is about $0.5^{\circ}$ [Horlings et al., 2008]. One actuator is activated in the direction of a patient's body tilt if it exceeds a tilt magnitude of $1^{\circ}$ (no.1 in figure B. 3 if the patient tilts forward). If the tilt angle increases in the same direction and exceeds $2.5^{\circ}$, the 2 adjacent actuators (no. 2 and 12 in figure B.3) are activated and the first actuator (no. 1) is deactivated. If the tilt angle increases and exceeds $4^{\circ}, 2$ adjacent and the middle actuators (no. 3, 1 and 11 in figure B.3) and the previous actuators (no. 2 and 12) are deactivated. Thus, the actuator which is activated between $1^{\circ}$ and $2.5^{\circ}$ of tilt indicates the tilt direction, whereas the number of actuators (the intensity of tactile stimulation (1, 2 or 3 actuators)) indicate the tilt angle. In this way the AVBF system can code body tilt in any direction. When the patient correctly responds to these actuators, they will be deactivated when the tilt angle drops below $1^{\circ}$ in any direction. However, if for example the patient responds by tilting backward and more than $1^{\circ}$ to the right, an actuator on the right side will be activated. 

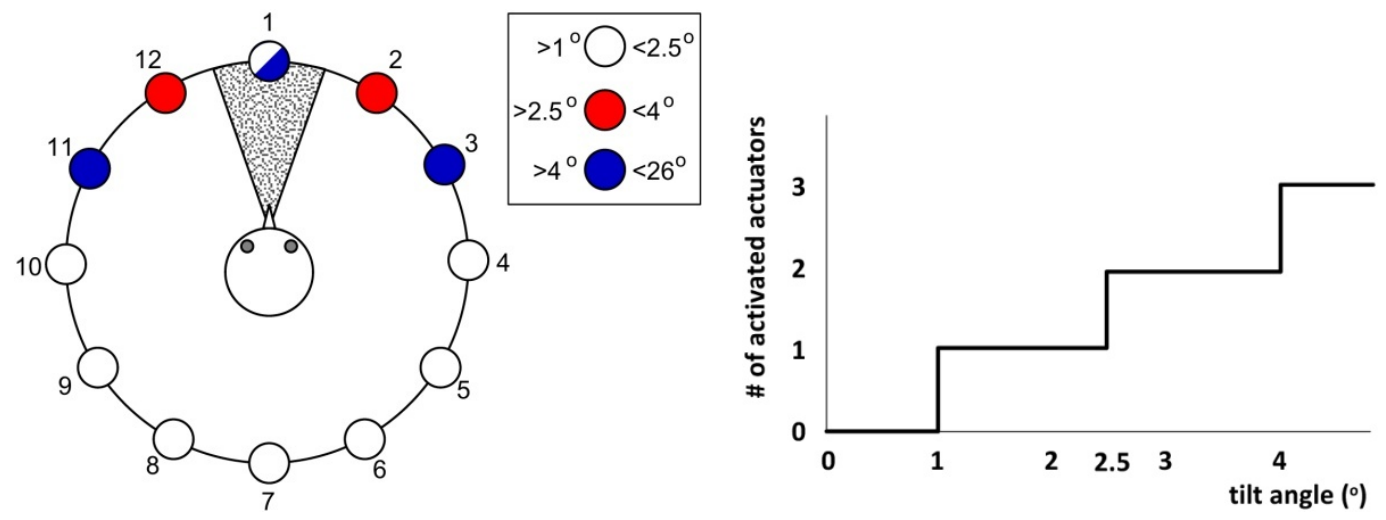

Figure B.3: Activation scheme of the actuator belt worn around the waist. The different marked actuators are activated at different tilt angles, relative to the reference vector, when the patient moves in the marked piece of the pie. In this case actuator no. 1 is activated at tilt angles $>1^{\circ}$ and $<2.5^{\circ}$, as well as $>4^{\circ}$.

\section{Patients}

20 patients participated in this study (10 males, 10 females, age 39-77 years) to assess the effect of the AVBF system on postural stability during gait. All patients had severe balance problems with frequent falls ( $>5$ times per year) and showed no responses to caloric irrigations $\left(30\right.$ and $44{ }^{\circ} \mathrm{C}$ ) and reduced or zero gains $(\leq 0.2)$ at sinusoidal stimulation of the horizontal and vertical canals on rotatory chair testing $(0.1 \mathrm{~Hz}$, $\mathrm{V}_{\max }=60 \% \mathrm{sec}$ ), pointing to a bilateral vestibular areflexia or severe bilateral vestibular hyporeflexia.

\section{Procedure}

Each patient had 5 minutes to familiarize with the AVBF system. Thereafter each patient practiced with the AVBF system for 15 minutes to learn how to use the system and to experience the relation between trunk or head movement and actuators. They were instructed to improve their balance using the vibrotactile biofeedback information, during stance and gait, both on a firm surface, with eyes open and closed. 
After practicing, gait was assessed with eyes open in all patients, and performance was scored using 3 standardized gait velocity tests in our clinical movement laboratory with a $9 \mathrm{~m}$ long and $1 \mathrm{~m}$ wide horizontal track using the Sybar videosystem:

1. slow tandem gait (1 step every 2 seconds (as described by Dozza et al. [Dozza et al., 2007])

2. fast tandem gait (more than 2 steps per second)

3. normal gait on $2 \mathrm{~cm}$ thick foam

Gait was recorded in the frontal (front and back) and sagittal plane using the Sybar videosystem [Harlaar et al., 2000]. An example video capture of a patient is shown in figure B.4. All gait tests were performed under 3 different conditions:

- noAVBF; without biofeedback

- $\mathrm{AVBF}_{\text {trunk: }}$ with biofeedback on the waist and sensor on the trunk

- $\mathrm{AVBF}_{\text {head }}$ : with biofeedback on the waist and sensor on the head

resulting in 9 different tests. Both the gait tests and biofeedback conditions were randomized.

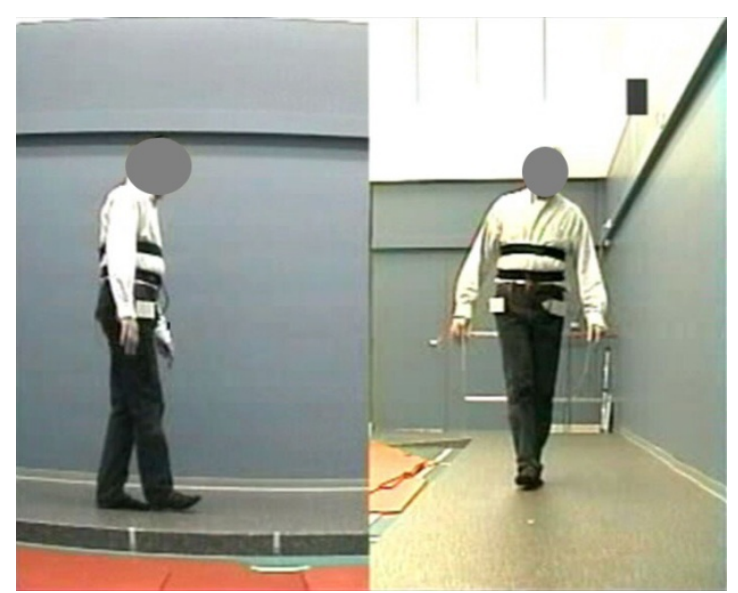

Figure B.4: An example video capture of a patient wearing the AVBF system and the sensor on the trunk, with the sagittal camera at the left and the frontal camera at the right. 


\section{Data analysis}

Blinded as to the vibrotactile feedback condition, balance during gait was scored independently by 3 expert observers (Y.J., H.V. and H.K.) based on the video recordings. Per standardized gait velocity test the observers identified the condition with best and worst balance during gait, upon which the 3 conditions were ranked on a 3-point scale (2=best, 1=medium, $0=$ =worst [Scholtes et al., 2007]). This resulted in an individual maximum score for one of the biofeedback conditions of 6 for each gait velocity test and 18 for the 3 gait tests combined. Wilcoxon's signed ranked test was used to determine the effect of the $A V B F$ system activated $\left(A V B F_{\text {trunk }}\right.$ and $\left.A V B F_{\text {head }}\right)$ versus the $A V B F$ system deactivated (noAVBF) for each gait velocity test and for the 3 gait tests combined.

As Bayesian analysis allows for a direct patient specific statement regarding the probability that a treatment was beneficial [Adamina et al., 2009], classical Bayesian probability statistics was used to determine a patient specific effect of the AVBF system, which was significant for a total score $>16$ or $<2$. The level of significance in all tests applied was $p<0.05$.

Interobserver reliability was assessed by using Intraclass Correlation Coefficients (ICCs), validated for use with multiple raters and calculated in a two-way random model based on absolute agreement, as described by Brunnekreef et al. [Brunnekreef et al., 2005]. After having fully tested a patient, the patient was asked in open question to comment on the functionality of the AVBF system and its effect on balance.

\subsection{Results}

The individual total gait scores are shown in table B.2. The inter-observer reliability of gait performance scoring was 0.68 (95\% Cl: 0.50-0.81), which was substantial. Patients' balance was significantly better in the $\mathrm{AVBF}_{\text {trunk }}$ condition than the noAVBF condition both during normal gait $(p=0.04)$ and fast tandem gait $(p=0.03)$. $A V B F_{\text {head }}$ and noAVBF were not significantly different in these gait tests. During slow tandem gait no significant differences could be shown. Over the total score, patients' balance during gait was significantly better in both the $\operatorname{AVBF}_{\text {trunk }}(p=0.01)$ and $\operatorname{AVBF}_{\text {head }}(p=0.03)$ condition than the noAVBF condition. Two patients (9 and 10) demonstrated significant individual improvements of balance during gait with the AVBF system activated as both performed worst without the AVBF system. 
Sixteen patients (80\%) commented that they felt more confident regarding their postural stability using the AVBF system and 14 of them were very interested to acquire a system. Balance improvement, increased confidence, independence, feeling more safe and the ability to perform multiple tasks in stance or during walking (e.g. talking and looking around) were reported. During a follow up consultation some patients mentioned that the use of the AVBF system even had improved their balance for several hours after the system had been removed.

\subsection{Discussion}

\section{Vibrotactile biofeedback system outcome}

We used videorecordings from the Sybar videosystem and scores from 3 expert observers to determine performance of balance during gait with and without vibrotactile biofeedback in 20 patients with a bilateral vestibular areflexia. Both during normal gait and fast tandem gait, the patients' balance was significantly better with biofeedback of the AVBF system on the waist and sensor on the trunk compared to no biofeedback. Additionally, using observational gait analysis as a means to score gait performance, we were able to identify significant individual improvements of balance during gait in 2 patients with the AVBF system activated.

These improvements are in line with work of Dozza and colleagues, who showed that stability during slow tandem gait improved using vibrotactile biofeedback [Dozza et al., 2007] in patients with unilateral vestibular loss. They also showed in their cross-over design that stability improved with repetition of tandem gait trials, thus indicating a learning effect of trial repetition, but that performance was consistently better in the trials with biofeedback than without biofeedback. In our study we controlled for a possible learning effect by randomizing the biofeedback conditions and gait velocities, thus the significant improvements in our patients can be attributed to effects of biofeedback. However, as we did not perform a placebo-controlled study [Janssen et al., 2010], the effects might also be due to the patient's belief [Yardley et al., 2001] or increased alertness [Basta et al., 2008, Dozza et al., 2007, Hegeman et al., 2005]. 
In contrast to Dozza et al. [Dozza et al., 2007] we were not able to show significant improvements in balance during slow tandem gait. This might be due to the task (slow tandem gait) being too difficult for bilateral vestibular areflexia patients to show balance improvements using vibrotactile biofeedback with limited practice time. Additionally Dozza et al. have shown a biofeedback effect in their patients with unilateral vestibular loss using a gait test with eyes closed, whereas our patients performed the gait tests with eyes open. An increased gait performance with eyes closed might be present in our gait tests as well, but more training with the AVBF system is then probably needed.

Hegeman et al. [Hegeman et al., 2005] found only little improvement in a group of patients with bilateral vestibular areflexia performing several gait tasks using auditory biofeedback, but their group may not have been a real at risk group in contrast to our (frequent falls), because none of their subjects had recently suffered a fall. Moreover, our results are in line with work of others showing that vestibular impaired patients are more stable using biofeedback in stance [Basta et al., 2008, Tyler et al., 2003, Danilov, 2004, Danilov et al., 2006, Danilov et al., 2007, Dozza et al., 2005b, Ernst et al., 2007, Goebel et al., 2009, Hegeman et al., 2005, Kentala et al., 2003].

\section{Sensor location}

Balance improvements during stance have been shown using a sensor on the trunk [Hegeman et al., 2005, Kentala et al., 2003, Ernst et al., 2007, Basta et al., 2008, Dozza et al., 2004, Dozza et al., 2005a, Dozza et al., 2005b, Dozza et al., 2007] as well as on the head [Tyler et al., 2003, Danilov, 2004, Danilov et al., 2006, Danilov et al., 2007, Goebel et al., 2009, Orlov et al., 2008, Wall et al., 2001]. We know of only one study about optimal sensor location in biofeedback [Janssen et al., 2010], which seems to be the head, but which also showed that the placebo effect of biofeedback might be substantial. The current study appears to indicate that the trunk seems to be the optimal location of the sensor (gait scores were significantly better for AVBF trunk versus noAVBF during gait tests 2 and 3 and never for $A V B F_{\text {head }}$ versus noAVBF), although not on an individual basis. So the optimal sensor location in biofeedback is yet to be examined in future studies and might even be individually determined. 


\subsection{Conclusions}

Although significant individual gait improvements were only shown in $10 \%$ of our patients, vibrotactile biofeedback increased confidence, independence, and a feeling of balance and safety in the majority of our patients. This was in agreement with Kentala et al. [Kentala et al., 2003]. Some patients even mentioned that the use of the vibrotactile biofeedback system had improved their balance for several hours after the device had been removed. This indicates the feasibility of the AVBF system to improve balance during gait and that the brain is able to include vibrotactile biofeedback in its predictive behaviour to avoid falls.

Some critical remarks after evaluating our results obtained so far and exploring the literature:

1. improvement of balance by biofeedback is shown in the minority of patients with balance problems [Ernst et al., 2007, Janssen et al., 2010]

2. training to optimise use of biofeedback seems to be essential [Basta et al., 2008, Ernst et al., 2007, Dozza et al., 2007, Danilov et al., 2006, Danilov et al., 2007]

3. adaptation effects do occur and reduce the impact of biofeedback in time during use [Ernst et al., 2007] but also prolong the impact after use [Danilov, 2004]

4. the placebo effect might be substantial [Dozza et al., 2007, Janssen et al., 2010]

Future studies should examine if patient performance in daily life or quality of life indeed increases using biofeedback. Compared to no feed back, biofeedback is a technique with a rewarding approach as it gives a positive feeling and motivates a patient to be more active and train challenging tasks. So, no doubt, by itself, biofeedback will be beneficial for training, rehabilitation and sensory substitution. 


\section{CHAPTER 3: SALIENT AND PLACEBO VIBROTACTILE FEEDBACK ARE EQUALLY EFFECTIVE IN REDUCING SWAY IN BILATERAL VESTIBULAR LOSS PATIENTS}

In this study we focus on the use of the AVBF system to increase postural stability in stance in patients with severe bilateral vestibular losses. A placebo-controlled evaluation of the effect of the AVBF system on static body sway and the optimal location of the sensor (trunk or head) will be presented. To our knowledge, no studies on the placebo effects of biofeedback have been published.

\subsection{Materials and methods}

\section{AVBF system activation scheme}

In this study, 3 activation modes were implemented in the AVBF system. In normal mode, the patient's tilt magnitude and tilt direction are translated into the activation of a specific actuator. One actuator is activated in the direction of a patient's body tilt if it exceeds a tilt magnitude of $2^{\circ}$. In this way the AVBF system can code body tilt in any direction and the actuator which is activated above $2^{\circ}$ of tilt indicates the tilt direction. When the patient correctly responds to the actuator, it will be deactivated when the tilt magnitude drops below $1.5^{\circ}$. The range between $1.5^{\circ}$ and $2^{\circ}$ was chosen to avoid abrupt changes in on and off switching of a specific actuator (in other words, hysteresis is induced). Thus, the dead zone has a size of $2^{\circ}$.

2 extra activation modes were implemented in the AVBF system as well to assess the placebo effect, which could be selected using a dedicated switch on the processor without having to set the reference vector. In full mode, if a patient's body tilt exceeds a tilt angle of $2^{\circ}$ in any direction, all actuators are activated, thus only the patient's tilt magnitude, and not tilt direction, is translated into the activation of all 12 actuators. In this way, a patient knows his body tilt exceeds a certain limit, but he doesn't know in which direction. When the patient corrects his body tilt back to below $1.5^{\circ}$, all 12 actuators are deactivated. In random mode, the AVBF processor decides pseudorandomly 5 times per second if an actuator will be switched on and if so, which one of the 12 actuators, thus neither the patient's tilt magnitude nor tilt direction are translated into the activation of a specific actuator. Even within the dead zone, an actuator is randomly switched on. Our patient's tended to test the AVBF system before 
every measurement and for that reason, in random mode the AVBF system functioned for the first 20 seconds as in normal mode.

Figure B.5 shows a schematic representation of the translation of body tilt angle to actuator activation in normal, full and random mode.

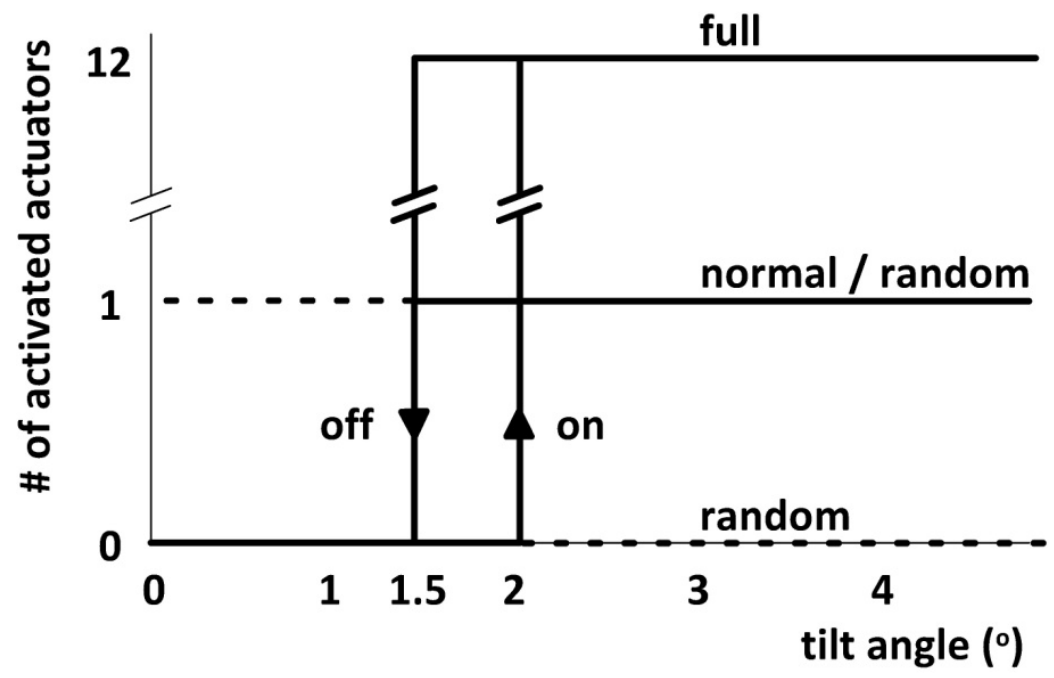

Figure B.5: Activation scheme of the actuator belt worn around the waist. In normal mode one actuator is activated in the direction of a subject's body tilt if it exceeds a tilt angle $2^{\circ}$ and deactivated when the tilt angle drops below $1.5^{\circ}$. In full mode, all 12 actuators are activated if body tilt exceeds a tilt angle $2^{\circ}$ and deactivated when the tilt angle drops below $1.5^{\circ}$. In random mode (dashed lines), the AVBF processor decides pseudo-randomly 5 times per second if an actuator will be switched on or off.

\section{Patients}

10 patients ( 7 males, 3 females, age 45-76 years) participated in this placebo-controlled study to assess the effect of the AVBF system on postural stability (AVBF group). 10 additional patients ( 5 males, 5 females, age 40-65 years) participated to assess interindividual and test-retest variability (reference group). All patients had severe balance problems with frequent falls ( $>5$ times per year) and showed no responses to caloric stimulation ( 30 and $44{ }^{\circ} \mathrm{C}$ ) of their lateral semicircular canals and reduced or zero gains $(\leq 0.2)$ at sinusoidal stimulation of the horizontal and vertical canals on rotatory chair testing $\left(0.1 \mathrm{~Hz}, \mathrm{~V}_{\max }=60 \% \mathrm{sec}\right)$, pointing to a bilateral vestibular areflexia or severe bilateral vestibular hyporeflexia. 


\section{Procedure}

The procedure for the AVBF group is schematically shown in table B.3.

Table B.3: Schematic representation of the procedure followed

\begin{tabular}{lllll}
\hline & surface & sensor location & AVBF mode & condition \\
\hline familiarization & firm & trunk & normal & \\
\hline practice & firm and foam & trunk and head & normal and full & \\
\hline body sway assessment & & - & - & 1 \\
\cline { 3 - 5 } & & & normal & 2 \\
& \multirow{2}{*}{ foam } & trunk & full & 3 \\
\cline { 3 - 4 } & & & random & 4 \\
\cline { 3 - 4 } & & head & normal & 5 \\
& & & full & 6 \\
& & & random & 7 \\
\hline
\end{tabular}

Each patient had 5 minutes to familiarize with the AVBF system. Thereafter each patient practiced with the AVBF system for 15 minutes to learn how to use the system and to experience the relation between trunk or head movement and actuators in both the normal mode and full mode. They were instructed to improve their balance using the vibrotactile biofeedback information, both on a firm surface as well as on a foam pad. Patients were informed about the application and evaluation of three activation modes. The feedback mechanism of the normal and full mode were explained in detail, whereas the patients were told that the third (random) mode was only slightly different from the normal mode and we tried to find the best out of the 3 modes to improve balance.

After practicing, body sway was assessed in all patients and performance was scored in 7 different conditions:

- without biofeedback (1)

- with biofeedback on the waist and sensor on the trunk in normal (2), full (3) and random (4) mode

- with biofeedback on the waist and sensor on the head in normal (5), full (6) and random (7) mode

Body sway was assessed using a force platform. Patients were instructed to stand bare feet on a $6 \mathrm{~cm}$ thick foam pad (Airex balance-pad) as still as possible for 45 second with 
the feet at hip width and the arms hanging along the sides. The first 5 seconds were performed with eyes open, to identify the patient's initial centre of pressure (COP), followed by 40 seconds with eyes closed. Each condition was measured in 2 trials, resulting in 14 measurements per patient. Each measurement started 15 seconds after the AVBF system was correctly activated (which includes setting the reference vector) and the activation mode was selected. This way, the biofeedback during the measurement when the eyes were closed was truly random in random mode.

Patients in the reference group were instructed to stand bare feet on a $6 \mathrm{~cm}$ thick foam pad (Airex balance-pad) as still as possible for 45 second with the feet at hip width and the arms hanging by the sides. The first 5 seconds were performed with eyes open, to identify the patient's initial COP, followed by 40 seconds with eyes closed. All patients performed 4 trials.

\section{Data and statistical analysis}

The (COP) was sampled at $50 \mathrm{~Hz}, 16$ bits, and related to the patient's body length to calculate the body sway angle (bs) in degrees in the horizontal plane

$b s=\arctan \{C O P / 0.56 \cdot$ body length $\}$

From bs swaypath, swayarea and mean sway (in both anteroposterior (AP) and mediolateral (ML) direction) were calculated per condition and averaged over the trials. Swaypath is defined as the length of the trajectory of the COP divided by the measurement time $(\mathrm{mm} / \mathrm{s})$. Swayarea is defined as the time integral of the area swept by the COP trajectory with respect to the initial COP, divided by the measurement time $\left(\mathrm{mm}^{2} / \mathrm{s}\right)$ [Baratto et al., 2002]. Mean sway is defined as the time average of the absolute value of the COP $(\mathrm{mm})$. In the reference group inter-individual variability and the testretest variability (the latter defined as standard deviation relative to the average), also often referred to as intra-individual variation, were calculated. In the AVBF group Wilcoxon's signed ranked test was used to determine the group effect of the AVBF system activated versus the condition with AVBF system deactivated. The changes in individual parameters were also determined per patient, relative to the condition without biofeedback (AVBF system deactivated). The level of significance in all tests applied was $p<0.05$. 
After having tested a patient, the patient was asked to rate the functionality of the AVBF system and its effect on balance on a scale from 0 to 10 .

\subsection{Results}

In the reference group, the test-retest variability was on average $9 \%$ for swaypath (95\% confidence interval (Cl): 1-17\%), $22 \%$ for swayarea (95\% Cl: 8-60 \%), 18\% for mean AP sway (95\% Cl: 6-51\%) and $21 \%$ for mean $\mathrm{ML}$ sway (95\% Cl: 6-67\%). The interindividual variability was $25 \%$ in swaypath, $45 \%$ in swayarea, $31 \%$ in mean AP sway and $35 \%$ in mean ML sway.

Because, of the 4 measures used in this study for body sway assessment, swaypath showed the smallest intra- and inter-individual variability in the reference group, swaypath was the only measure used for analysis in the AVBF group. A significant decrease in swaypath was identified when it exceeded $17 \%$. The relative decreases in swaypath per patient comparing the AVBF system activated with the AVBF system deactivated are shown in figure B.6 and table B.4. Table B.4 also shows the significant swaypath changes.

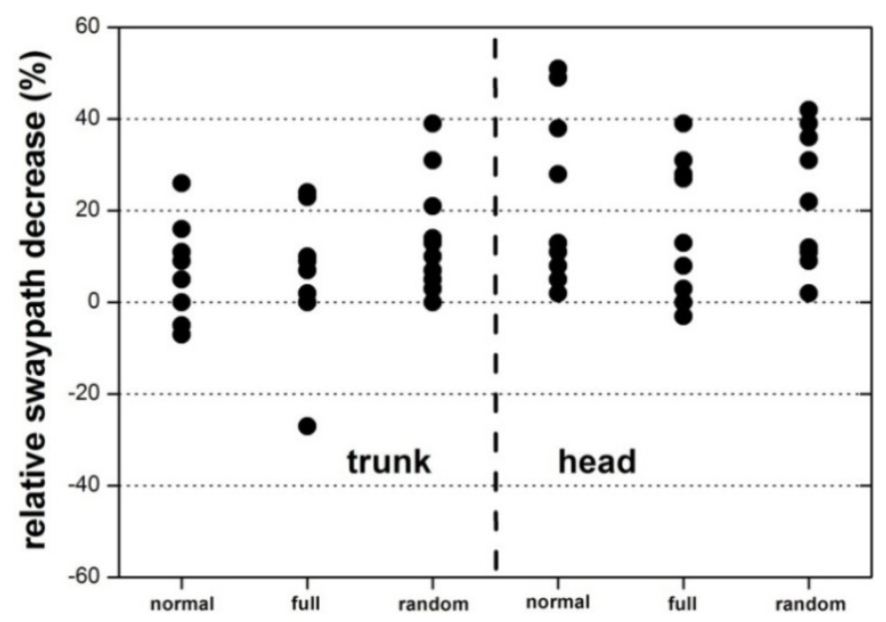

Figure B.6: Relative decreases in swaypath per patient using the AVBF system (on foam, eyes closed, feet at hip width and without footwear) in 2 (sensor location) conditions and in the 3 different activation modes (normal, full, random). A change in swaypath larger than $17 \%$ is significant. Absolute swaypath values in these patients were on average $122 \mathrm{~mm} / \mathrm{s}$ (95 \% Cl: 71$205 \mathrm{~mm} / \mathrm{s}$ ) 
Table B.4: Relative decreases in swaypath per patient using the AVBF system, sorted by the decrease in swaypath with the AVBF system activated in normal mode and sensor on the head. Trunk = sensor on the trunk, head = sensor on the head, normal = AVBF activation mode normal, full = AVBF activation mode full, random = AVBF activation mode random, see text for details. The cells marked in grey indicate the significant swaypath differences.

\begin{tabular}{|c|c|c|c|c|c|c|c|c|c|}
\hline \multirow[t]{2}{*}{ patient } & \multirow[t]{2}{*}{ age } & \multirow[t]{2}{*}{ gender } & \multicolumn{3}{|c|}{ Trunk (\%) } & \multicolumn{3}{|c|}{ Head (\%) } & \multirow{2}{*}{$\begin{array}{l}\text { AVBF system } \\
\text { rating }\end{array}$} \\
\hline & & & normal & full & random & normal & full & random & \\
\hline 10 & 62 & $\mathrm{~m}$ & 16 & 23 & 39 & 51 & 28 & 42 & 6 \\
\hline 6 & 76 & $\mathrm{~m}$ & 26 & 24 & 31 & 49 & 31 & 36 & 4 \\
\hline 1 & 60 & $\mathrm{~m}$ & 11 & 9 & 14 & 38 & 39 & 39 & 9 \\
\hline 8 & 57 & $\mathrm{~m}$ & -7 & 0 & 5 & 28 & 27 & 31 & 6 \\
\hline 9 & 64 & $\mathrm{~m}$ & 0 & 2 & 7 & 13 & 8 & 9 & 7 \\
\hline 2 & 69 & $\mathrm{~m}$ & 0 & 9 & 21 & 11 & -3 & 11 & 4 \\
\hline 7 & 67 & $f$ & -5 & -27 & 3 & 11 & 3 & 2 & 7 \\
\hline 3 & 56 & $\mathrm{~m}$ & 16 & 10 & 13 & 8 & 13 & 22 & 7 \\
\hline 5 & 75 & $f$ & 5 & 7 & 0 & 5 & 0 & 9 & 7 \\
\hline \multirow[t]{2}{*}{4} & 45 & $f$ & 9 & 10 & 10 & 2 & 8 & 12 & 8 \\
\hline & & average & 7 & 7 & 17 & 22 & 15 & 23 & 6.5 \\
\hline$p$ & & & .05 & .07 & .01 & .005 & .01 & .01 & \\
\hline
\end{tabular}

With biofeedback on the waist and sensor on the trunk, a maximal decrease in swaypath of $39 \%$ was shown. A decrease in swaypath was shown in 6 patients in normal mode (7 $\%$ on average), in 8 patients in full mode ( $7 \%$ on average) and in 9 patients in random mode ( $17 \%$ on average, $p=0.01$ ). In patient no. 6 a significant decrease in swaypath ( 26 $\%)$ was shown in normal mode. A significant decrease in body sway in full and random mode ( 24 and $31 \%$ respectively) was also shown in this patient. In patient no. 10 a significant decrease in swaypath was shown in full and random mode ( 23 and $39 \%$ respectively), not in normal mode (16\%). In both these patients (no. 10 and 6) the decrease in swaypath in random mode was larger than in full and normal mode.

With biofeedback on the waist and sensor on the head, a maximal decrease in swaypath of $51 \%$ was shown. A decrease in swaypath was shown in all 10 patients in normal mode ( $22 \%$ on average, $p=0.005)$, in 8 patients in full mode ( $15 \%$ on average, $p=0.01$ ) and in 10 patients in random mode ( $23 \%$ on average, $p=0.01$ ). In 4 patients (no. 10, 6, 1 and 8) a significant decrease in swaypath was shown in normal mode. A significant 
decrease in swaypath in full and random mode was also shown in these patients. The decrease in swaypath in random mode was larger, but not significant, than in full mode.

Patients rated the functionality of the AVBF system and its effect on balance on average 6.5 (range 4-9).

\subsection{Discussion}

\section{Vibrotactile biofeedback system outcome}

In this study swaypath was used as outcome parameter, because it showed the smallest intra- and inter-individual variability. Moreover it was recently shown to be particularly valuable in clinical practice [Baratto et al., 2002, Raymakers et al., 2005]. A significant decrease in swaypath was present in 4 of our patients with bilateral vestibular areflexia using vibrotactile biofeedback. This in line with other studies showing that vestibular impaired patients are more stable using biofeedback in stance [Basta et al., 2008, Tyler et al., 2003, Danilov, 2004, Danilov et al., 2006, Danilov et al., 2007, Dozza et al., 2005b, Ernst et al., 2007, Goebel et al., 2009, Hegeman et al., 2005, Kentala et al., 2003]. Moreover, using a reference group to quantify test-retest variability we were able to identify individual stance improvements.

\section{Impact of feedback versus alerting}

The comparison of the vibrotactile biofeedback system activated versus deactivated was performed in 3 activation modes (normal, full and random). Because balance improvements in normal mode were only observed when balance had also improved in full and random mode, the improvements were, at least partially, caused by other effects than tilt magnitude and tilt direction biofeedback. These effects could be 1) training because vibrotactile stimulation might help to optimize brain plasticity and improves motor performance [Dozza et al., 2007, Ernst et al., 2007], 2) the patient's belief [Yardley et al., 2001] or 3) increased alertness [Basta et al., 2008, Dozza et al., 2007, Hegeman et al., 2005], as it continuously gives specific cues to reduce postural sway [Asseman et al., 2007]. Speculative, elegant study protocols to show the true effect of biofeedback are crucial, because performing more measurements (and thus more practicising in a certain condition) will show a training effect and switching off the biofeedback will decrease patient's alertness and will increase the placebo effect of these biofeedback systems. 


\section{Sensor location}

With biofeedback on the waist and the sensor on the head 4 patients showed a significant improvement in swaypath (in all 3 activation modes), whereas with sensor on the trunk only 1 patient showed a significant improvement in swaypath in all 3 activation modes and another patient only in two activation modes. Thus the head seems to be the optimal location of the sensor. To our knowledge no studies on the optimal sensor location in biofeedback have been published [Wall et al., 2002], but improvements have been shown using a sensor on the trunk [Hegeman et al., 2005, Kentala et al., 2003, Ernst et al., 2007, Basta et al., 2008, Dozza et al., 2004, Dozza et al., 2005a, Dozza et al., 2005b, Dozza et al., 2007] as well as on the head [Tyler et al., 2003, Danilov, 2004, Danilov et al., 2006, Danilov et al., 2007, Goebel et al., 2009, Orlov et al., 2008, Wall et al., 2001]. Future studies are needed to examine the individual optimal sensor location.

\subsection{Conclusions}

Vibrotactile biofeedback reduces body sway in some patients with severe balance problems with frequent falls, indicating that the brain is able to include vibrotactile biofeedback in its predictive behaviour to improve balance. But the major question is why? And is there any true effect of biofeedback beyond a placebo effect? Several work suggest that there is a fundamental effect, because biofeedback consistently increased postural stability, beyond the effects of practice alone [Danilov et al., 2007, Dozza et al., 2007, Ernst et al., 2007].

Some critical remarks after evaluating our results and exploring the literature can be made:

1. improvement of balance by biofeedback is shown in the minority of patients with balance problems - this study and [Ernst et al., 2007]. The impact of biofeedback might vary among patients, because of differences in 1) the severity of the vestibular deficit and its effect on postural control, 2) the dependence on vestibular input for postural control compared to the other senses, 3) patient motivation and 4) the ability to learn how to switch to vibrotactile feedback of body sway and to interpret all sensory information to keep body sway within stability limits [Asseman et al., 2007] 
2. training to optimise use of biofeedback seems to be essential [Basta et al., 2008, Ernst et al., 2007, Dozza et al., 2007, Danilov et al., 2006, Danilov et al., 2007]

3. adaptation effects do occur and reduce the impact of biofeedback in time during use [Ernst et al., 2007] but also prolong the impact after use [Danilov, 2004]. This would suggest that repetitive applications of relatively short duration of vibrotactile biofeedback might improve balance and orientation and that continuous use of such a device might not be necessary

4. the placebo effect might be substantial - this study and [Dozza et al., 2007]

The even more important question is, if patient performance in daily life or quality of life will indeed increase by biofeedback. It will at least have a benefit in patient motivation to be more active, to train in challenging tasks and to learn more about postural sway and its dependence on daily life activities. Moreover, because there is a need for training and rehabilitation, biofeedback is a rewarding approach as it gives a positive feeling compared to the situation when no feedback has been given at all during a task. Therefore we continue the development and evaluation of a vibrotactile biofeedback system for a broad clinical application, but we are aware of the fact that our study suggests that a major impact of AVBF application is related to support training and sensory substitution. 


\section{References}

Adamina, M., Tomlinson, G. \& Guller, U. (2009) Bayesian statistics in oncology: a guide for the clinical investigator. Cancer.

Alahakone, A. U. \& Senanayake, S. M. N. A. (2010) A real-time system with assistive feedback for postural control in rehabilitation. IEEE/ASME Transactions on Mechatronics, 15, 226233.

Asseman, F., Bronstein, A. M. \& Gresty, M. A. (2007) Using vibrotactile feedback of instability to trigger a forward compensatory stepping response. J Neurol, 254, 1555-61.

Balter, S. G., Stokroos, R. J., De Jong, I., Boumans, R., Van De Laar, M. \& Kingma, H. (2004) Background on methods of stimulation in galvanic-induced body sway in young healthy adults. Acta Otolaryngol, 124, 262-71.

Baratto, L., Morasso, P. G., Re, C. \& Spada, G. (2002) A new look at posturographic analysis in the clinical context: sway-density versus other parameterization techniques. Motor Control, 6, 246-70.

Basta, D., Singbartl, F., Todt, I., Clarke, A. \& Ernst, A. (2008) Vestibular rehabilitation by auditory feedback in otolith disorders. Gait Posture, 28, 397-404.

Bisdorff, A. R., Wolsley, C. J., Anastasopoulos, D., Bronstein, A. M. \& Gresty, M. A. (1996) The perception of body verticality (subjective postural vertical) in peripheral and central vestibular disorders. Brain, 119 ( Pt 5), 1523-34.

Bringoux, L., Schmerber, S., Nougier, V., Dumas, G., Barraud, P. A. \& Raphel, C. (2002) Perception of slow pitch and roll body tilts in bilateral labyrinthine-defective subjects. Neuropsychologia, 40, 367-72.

Brunnekreef, J. J., Van Uden, C. J., Van Moorsel, S. \& Kooloos, J. G. (2005) Reliability of videotaped observational gait analysis in patients with orthopedic impairments. BMC Musculoskelet Disord, 6, 17.

Danilov, Y. (2004) Effects of electrotactile head-based feedback on subjects with bilateral vestibular dysfunction. Proc Bárány Society.

Danilov, Y. P., Tyler, M. E., Skinner, K. L. \& Bach-Y-Rita, P. (2006) Efficacy of electrotactile vestibular substitution in patients with bilateral vestibular and central balance loss. Conf Proc IEEE Eng Med Biol Soc, Suppl, 6605-9.

Danilov, Y. P., Tyler, M. E., Skinner, K. L., Hogle, R. A. \& Bach-Y-Rita, P. (2007) Efficacy of electrotactile vestibular substitution in patients with peripheral and central vestibular loss. J Vestib Res, 17, 119-30.

Della Santina, C., Migliaccio, A. \& Patel, A. (2005) Electrical stimulation to restore vestibular function development of a 3-d vestibular prosthesis. Conf Proc IEEE Eng Med Biol Soc, 7, 7380-5.

Dennerlein, J. T., Millman, P. A. \& Howe, R. D. (1997) Vibrotactile feedback for industrial telemanipulators. ASME IMECE.

Dozza, M., Chiari, L., Chan, B., Rocchi, L., Horak, F. B. \& Cappello, A. (2005a) Influence of a portable audio-biofeedback device on structural properties of postural sway. J Neuroeng Rehabil, 2, 13. 
Dozza, M., Chiari, L. \& Horak, F. B. (2004) A portable audio-biofeedback system to improve postural control. Conf Proc IEEE Eng Med Biol Soc, 7, 4799-802.

Dozza, M., Chiari, L. \& Horak, F. B. (2005b) Audio-biofeedback improves balance in patients with bilateral vestibular loss. Arch Phys Med Rehabil, 86, 1401-3.

Dozza, M., Wall, C., $3^{\text {rd }}$, Peterka, R. J., Chiari, L. \& Horak, F. B. (2007) Effects of practicing tandem gait with and without vibrotactile biofeedback in subjects with unilateral vestibular loss. J Vestib Res, 17, 195-204.

Ernst, A., Singbartl, F., Basta, D., Seidl, R. O., Todt, I. \& Eisenschenk, A. (2007) Short-term rehabilitation of patients with posttraumatic otolith disorders by auditory feedback training: a pilot study. J Vestib Res, 17, 137-44.

Goebel, J. A., Sinks, B. C., Parker, B. E., Jr., Richardson, N. T., Olowin, A. B. \& Cholewiak, R. W. (2009) Effectiveness of head-mounted vibrotactile stimulation in subjects with bilateral vestibular loss: a phase 1 clinical trial. Otol Neurotol, 30, 210-6.

Harlaar, J., Redmeijer, R. A., Tump, P., Peters, R. \& Hautus, E. (2000) The SYBAR system: integrated recording and display of video, EMG, and force plate data. Behav Res Methods Instrum Comput, 32, 11-6.

Hegeman, J., Honegger, F., Kupper, M. \& Allum, J. H. (2005) The balance control of bilateral peripheral vestibular loss subjects and its improvement with auditory prosthetic feedback. J Vestib Res, 15, 109-17.

Hemami H., Weimer F.C., Robinson C.S., Stockwell C.W., Cvetkovic V.S. (1978) Biped stability considerations with vestibular models. IEEE Trans. on Autom. Control, AC-23, 1074-79

Hirvonen, T. P., Aalto, H. \& Pyykko, I. (1997) Stability limits for visual feedback posturography in vestibular rehabilitation. Acta Otolaryngol Suppl, 529, 104-7.

Horlings, C. G., Kung, U. M., Bloem, B. R., Honegger, F., Van Alfen, N., Van Engelen, B. G. \& Allum, J. H. (2008) Identifying deficits in balance control following vestibular or proprioceptive loss using posturographic analysis of stance tasks. Clin Neurophysiol, 119, 2338-46.

Janssen, M., Stokroos, R., Aarts, J., Van Lummel, R. \& Kingma, H. (2010) Salient and placebo vibrotactile feedback are equally effective in reducing sway in bilateral vestibular loss patients. Gait Posture, 31, 213-7.

Jones, L. A. \& Sarter, N. B. (2008) Tactile displays: guidance for their design and application. Hum Factors, 50, 90-111.

Kentala, E., Vivas, J. \& Wall, C., $3^{\text {rd }}$ (2003) Reduction of postural sway by use of a vibrotactile balance prosthesis prototype in subjects with vestibular deficits. Ann Otol Rhinol Laryngol, 112, 404-9.

Kuo A.D. (1995) An optimal control model for analyzing human postural balance. IEEE Trans on Biomed Eng, 42, 87-101

Lewis, R. F., Gong, W., Ramsey, M., Minor, L., Boyle, R. \& Merfeld, D. M. (2002) Vestibular adaptation studied with a prosthetic semicircular canal. J Vestib Res, 12, 87-94.

Mahns, D. A., Perkins, N. M., Sahai, V., Robinson, L. \& Rowe, M. J. (2006) Vibrotactile frequency discrimination in human hairy skin. J Neurophysiol, 95, 1442-50. 
Nashner, L. M., Shupert, C. L., Horak, F. B. \& Black, F. O. (1989) Organization of posture controls: an analysis of sensory and mechanical constraints. Prog Brain Res, 80, 411-8; discussion 395-7.

Okamura, A. M., Dennerlein, J. T. \& Howe, R. D. (1998) Vibration Feedback Models for Virtual Environments. Proc IEEE Int Conf Robotics Automation, 3, 2485-2490.

Orlov, I. V., Stolbkov, Y. K. \& Shuplyakov, V. C. (2008) Effects of artificial feedback to the vestibular input on postural instability induced by asymmetric proprioceptive stimulation. Neurosci Behav Physiol, 38, 195-201.

Peterka, R. J., Wall, C., $3^{\text {rd }}$ \& Kentala, E. (2006) Determining the effectiveness of a vibrotactile balance prosthesis. J Vestib Res, 16, 45-56.

Ram, S. \& Sharf, J. (1998) The People Sensor: A Mobility Aid for the Visually Impaired. IEEE Wearable Computers.

Raymakers, J. A., Samson, M. M. \& Verhaar, H. J. (2005) The assessment of body sway and the choice of the stability parameter(s). Gait Posture, 21, 48-58.

Scholtes, V. A., Dallmeijer, A. J., Knol, D. L., Speth, L. A., Maathuis, C. G., Jongerius, P. H. \& Becher, J. G. (2007) Effect of multilevel botulinum toxin a and comprehensive rehabilitation on gait in cerebral palsy. Pediatr Neurol, 36, 30-9.

Scinicariello, A. P., Eaton, K., Inglis, J. T. \& Collins, J. J. (2001) Enhancing human balance control with galvanic vestibular stimulation. Biol Cybern, 84, 475-80.

Tyler, M., Danilov, Y. \& Bach, Y. R. P. (2003) Closing an open-loop control system: vestibular substitution through the tongue. J Integr Neurosci, 2, 159-64.

Wall, C., $3^{\text {rd }}$, Kos, M. I. \& Guyot, J. P. (2007) Eye movements in response to electric stimulation of the human posterior ampullary nerve. Ann Otol Rhinol Laryngol, 116, 369-74.

Wall, C., $3^{\text {rd }}$, Merfeld, D. M., Rauch, S. D. \& Black, F. O. (2002) Vestibular prostheses: the engineering and biomedical issues. J Vestib Res, 12, 95-113.

Wall, C., $3^{\text {rd }}$, Weinberg, M. S., Schmidt, P. B. \& Krebs, D. E. (2001) Balance prosthesis based on micromechanical sensors using vibrotactile feedback of tilt. IEEE Trans Biomed Eng, 48, 1153-61.

Yardley, L., Beech, S. \& Weinman, J. (2001) Influence of beliefs about the consequences of dizziness on handicap in people with dizziness, and the effect of therapy on beliefs. $J$ Psychosom Res, 50, 1-6.

Yates, B. J. \& Bronstein, A. M. (2005) The effects of vestibular system lesions on autonomic regulation: observations, mechanisms, and clinical implications. J Vestib Res, 15, 119-29. 


\section{Part C}

SPATIAL ORIENTATION
In this part a) normative data for the perception threshold for rotation, tilt and translation and b) the perception threshold for rotation in patients with a history pointing to a possible vestibular deficit were determined. Subjects were submitted to a) deceleration step stimuli during continuous subthreshold acceleration rotation, resulting in rotation sensations in the opposite direction of the actual rotation and b) to 1) unilateral and bilateral eccentric rotations (centrifugation), 2) whole body translatory decelerations opposite to the movement direction while seated on a linear sled and 3) discrete slow velocity platform tilts. Subjects were instructed to verbally indicate a) a change in rotation direction and b) the perceived direction of tilt or translation.

Perception thresholds for rotation could be assessed in a reproducible way, but 13 out of 18 patients with a partial or complete unilateral or bilateral caloric hypofunction had normal rotation perception thresholds. Thus the perception threshold for rotation might have a complimentary value in diagnostics and be more specifically related to vestibular deficits than the classical test.

Our results from unilateral centrifugation indicate a threshold for body tilt perception of about $2^{\circ}$, which to our interpretation mainly depends on otolithic function. Tilt sensations during eccentric rotations appear to be a promising tool for the evaluation of utricular dysfunction. Tilt perception during whole body decelerations and discrete platform tilts mainly depends on somatosensory information, showing the dominant role of the somatosensory system for the perception of body orientation. 
This part would not have been possible without the work of my graduate students Rik Hanssen and Mieke Bijveld, the help from my trainees Xander Janssen, Nico Vanhove, Pieter van Deutekom, Lia Vugts, Martijn Wolters, Twan Bloebaum, Wesley van der Ven, Marc Lauvenberg and Bram Geerets, the technical support from Iwan de Jong, René Hermans, Simon Biermans, Paul Laeven, Erik Brands and Harold Walraven and the technicians Ellen Rikers, Sophie Paredis and Marie-Cécile Gerards from the vestibular department.

Based on

Janssen $M$, van der Ven $W$, Wolters $M$ and Kingma $H$. Clinical application of perception threshold of horizontal plane rotation. J Vest Res 2011, submitted and Janssen $M$, Lauvenberg $M$, van der Ven $W$, Bloebaum $T$ and Kingma $H$. Perception Threshold for Tilt. Otol Neurotol 2011 


\section{CHAPTER 4: CLINICAL APPLICATION OF PERCEPTION THRESHOLD OF HORIZONTAL PLANE ROTATION}

\subsection{Introduction}

Angular head accelerations induce a deflection of the cupula, which codes head velocity in the physiological frequency domain [Melvill-Jones, 1979] and among others leads to compensatory eye movements, the vestibular ocular reflex (VOR). Quantification of the VOR under different conditions is the common way for the diagnosis of possible vestibular dysfunction. Angular accelerations lead to a sensation of rotation, and visual illusion of motion (oculogyral) even when no eye movements are induced [Benson et al., 1989]. Rotation perception has been quantified using a tachometer to indicate the sensed turning velocity [Grunfeld et al., 2000], by self-controlled reorienting [Metcalfe and Gresty, 1992, von Brevern et al., 1997] or by indicating the direction of rotation [Grabherr et al., 2008, Clark and Stewart, 1970]. The latter has even led to the introduction of a "vestibulogram" [Grabherr et al., 2008], the vestibular equivalent of an audiogram quantifying thresholds of rotation perception as a function of frequency of sinusoidal stimuli. Because of the inverse relationship between the duration of constant angular accelerations and their detection threshold [Clark et al., 1980], it has been suggested to use the product of acceleration and its duration (Mulder product expressed in $\%$ ) to quantify thresholds for short duration stimuli. This product is theoretically constant for constant angular acceleration stimuli with a duration between 0.5 and $5 \mathrm{~s}$ (frequencies between 0.1 and $1 \mathrm{~Hz}$ ). This is due to the response characteristics of the semicircular canals: after a step in angular acceleration the cupula deflection is exponential with a time constant of about 10 seconds [Melvill-Jones, 1979, Guedry, 1974], which can initially (first 5 seconds) be approximated with linear deflection after initiation of the acceleration step, as shown in figure C.1. For stimuli longer than $5 \mathrm{~s}$ (frequency $<0.1 \mathrm{~Hz}$ ) the threshold will increase due to the exponential cupula response to an acceleration step [Rodenburg et al., 1981, Benson et al., 1989]. Both the acceleration threshold as well as the Mulder product threshold show a wide range in healthy subjects: $0.035-4 \% \mathrm{~s}^{2}$ [Clark, 1967] and $0.38-2.84 \%$ [Grabherr et al., 2008] respectively. 
Several studies suggest that the proprioceptive threshold for movements is lower than vestibular thresholds [Benson et al., 1986, Clark, 1967], which puts a question mark at the normative data for the vestibular rotation thresholds determined in the literature. Especially, very short stimuli, e.g. below $0.5 \mathrm{~s}$ (above $1 \mathrm{~Hz}$ ) will also give tactile cues, and therefore might lead to lower thresholds for rotation perception and not indicate real vestibular sensitivity.
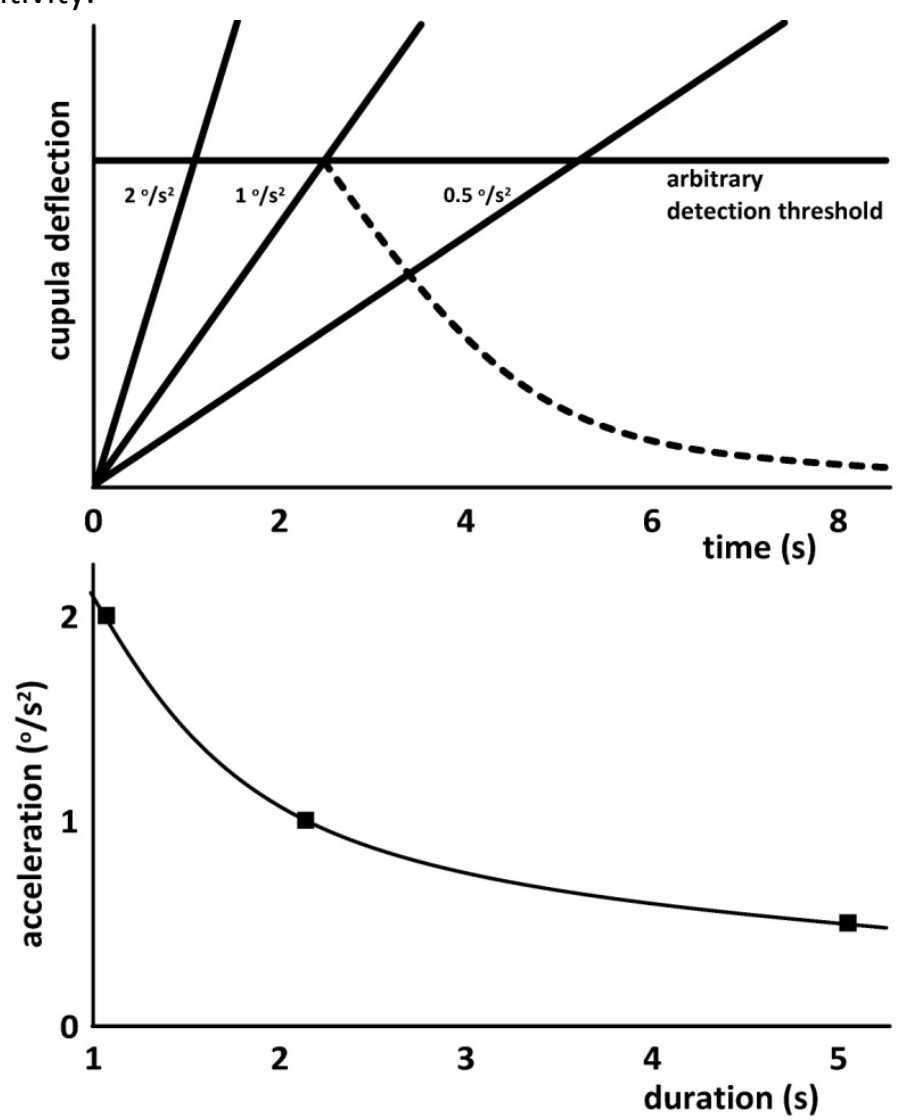

Figure C.1: (a) Model description of cupula deflection as a function of time (solid lines) after a step in acceleration from $0 \% \mathrm{~s}^{2}$ to $0.5,1.0$ and $2.0 \% \mathrm{~s}^{2}$ at $t=0 \mathrm{~s}$. An arbitrary fixed detection threshold, results in detection of rotation after 5.11, 2.23 and 1.05 seconds - a threshold of 2.55, 2.23 and $2.1 \%$ respectively. The dashed line reflects the cupula relaxation after a step in acceleration back to $0 \% \mathrm{~s}^{2}$ at $t=2.23 \mathrm{~s}$. (b) the inverse relationship between stimulus duration and angular acceleration for a fixed threshold. For stimuli of $5 \mathrm{~s}$ or longer the actual threshold (the product of the acceleration and its duration) is $\geq 20 \%$ lower, due to the exponential cupula response to an acceleration step 
Although several studies on rotation perception quantification have been performed, only a few studies have shown clinical data for rotation perception thresholds. Metcalfe and Gresty showed that subjects without labyrinths failed to respond because they could not sense the stimuli and failed to stop themselves rotating by self-controlled reorienting [Metcalfe and Gresty, 1992]. Patients with unilateral absence of vestibular function who were tested within a month after vestibular nerve section gave similar responses as the subjects without labyrinths. However, within several months all changed to bidirectional normal responses. Similar results were reported by Kanayama et al. [Kanayama et al., 1995] and by von Brevern et al. [von Brevern et al., 1997]. Roggeveen and Nijhof showed that patients with Ménière's syndrome all had increased thresholds for turning sensation [Roggeveen and Nijhoff, 1956]. Okada et al. showed that congenital nystagmus patients have a shortened vestibular sensation of rotation [Okada et al., 1999]. Von Brevern et al. additionally showed that patients with posterior canal plugging systematically underestimated the magnitude of their stimuli.

\section{This study}

The stimuli generally applied for threshold detection are composed of motion profiles starting from and/or stopping at zero velocity which not only stimulate the vestibular end organs but also give tactile cues, especially at higher frequencies [Clark, 1967]. These stimuli, being triangular or sinusoidal, also contain a certain periodicity which is by nature predictable and might lower the threshold [Benson et al., 1989]. It is easier to feel a difference between rhythmically alternating periods of stimulation and no stimulation than to recognize a small stimulus during a prolonged stimulation time. To reduce the impact of non-vestibular cues for rotation we 1) masked sounds, 2) eliminated air flow detection, and 3) used deceleration step stimuli [Clark and Stewart, 1970] during continuous subthreshold acceleration rotation. In this way, as a new motion profile paradigm centered on slowing down, specific vestibular rotation perceptions opposite to the actual rotation direction are induced. Subjects were instructed to indicate the direction of the rotation stimulus (driven by the deceleration step), and not sensed velocity or orientation as these are estimated based on central integration of vestibular nerve signals.

Our goal was to provide normative data for the perception threshold for rotation in the horizontal plane and to determine this threshold in patients with a history clearly pointing to a vestibular deficit. In 18 out of the 20 patients the vestibular deficit was confirmed by an abnormal torsion swing (sinusoidal chair rotation) and/or caloric test. 


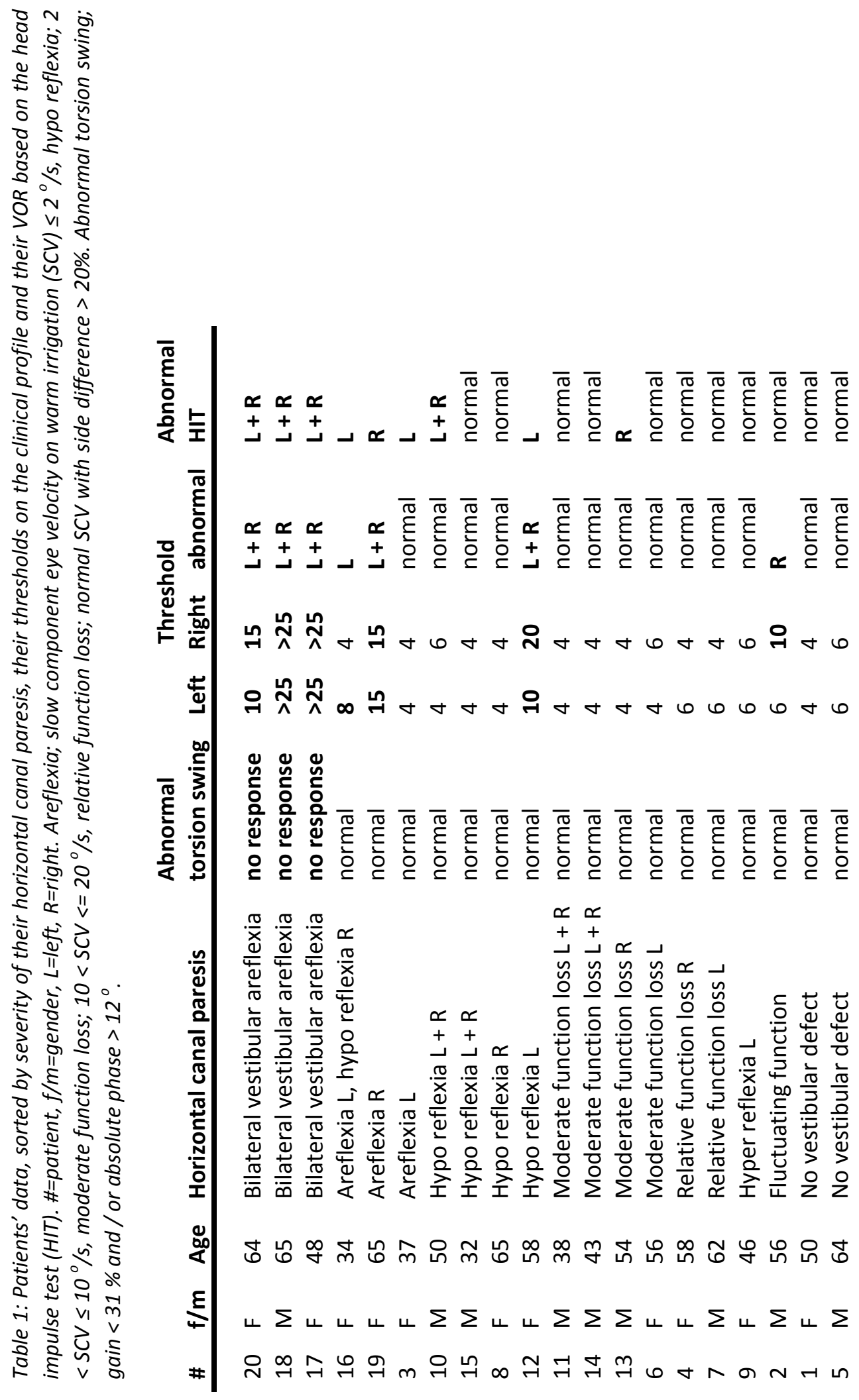




\subsection{Methods}

\section{Healthy subjects}

17 healthy subjects (10 male, 7 female, 19 - 31 years) without any history or evidence of any ophthalmologic or neuro-otological disorder participated in this study. All subjects participated on a voluntary basis after giving their informed consent. Subjects were seated in a chair (Nydiak 500, Ekida $\mathrm{GmbH}$ ) in our vestibular clinic with a 3-points seatbelt, feet secured, each hand around a bar fixed to the armrest of the chair, eyes closed and masked, headphones mounted to mask auditory cues for spatial orientation and to communicate with the operator. Chair accelerations could be produced in 0.01 $\% / \mathrm{s}^{2}$ steps at the low velocities used in this study, making it possible to produce changes in acceleration with a rise time in the order of $0.01 \mathrm{~s}$ [Clark and Stewart, 1970]. The chair was rotated about an earth-vertical (yaw) axis in a light-tight room, which further minimized possible visual and auditory cues. To orient the horizontal canals with the plane of rotation, subjects flexed their neck slightly [von Brevern et al., 1997]. Subjects were instructed to keep their head in this specific position, which was constantly monitored by the operator via an infra red sensitive camera.

The stimuli opposed the continuous rotation direction of the chair, thus opposing possible perception of the actual rotation direction and at the same time minimizing possible tactile cues such as the start of rotation and wind on the skin.

\section{Patients}

20 patients ( 9 male, 11 female, $32-65$ years) with a clinical history clearly pointing to a vestibular deficit participated in this study. All patients were examined by a neurootologist and underwent standard vestibular tests (spontaneous, gaze, smooth pursuit, saccades, optokinetic, torsion swing $0.1 \mathrm{~Hz}$, peak velocity $75 \%$ s and caloric tests of 30 and $44{ }^{\circ} \mathrm{C}$ ). In all patients oculomotor tests were normal and in 17 patients the torsion swing test was normal (gain $>31 \%$ and / or absolute phase $<12 \%$ ). Only 3 patients with a bilateral vestibular areflexia had an abnormal torsion swing. Patients were classified based on the outcome of the caloric tests (see table C.1). In one patient, no. 2, the caloric test had been performed multiple times showing fluctuating caloric responses. Clinical details are shown in table C.1. 

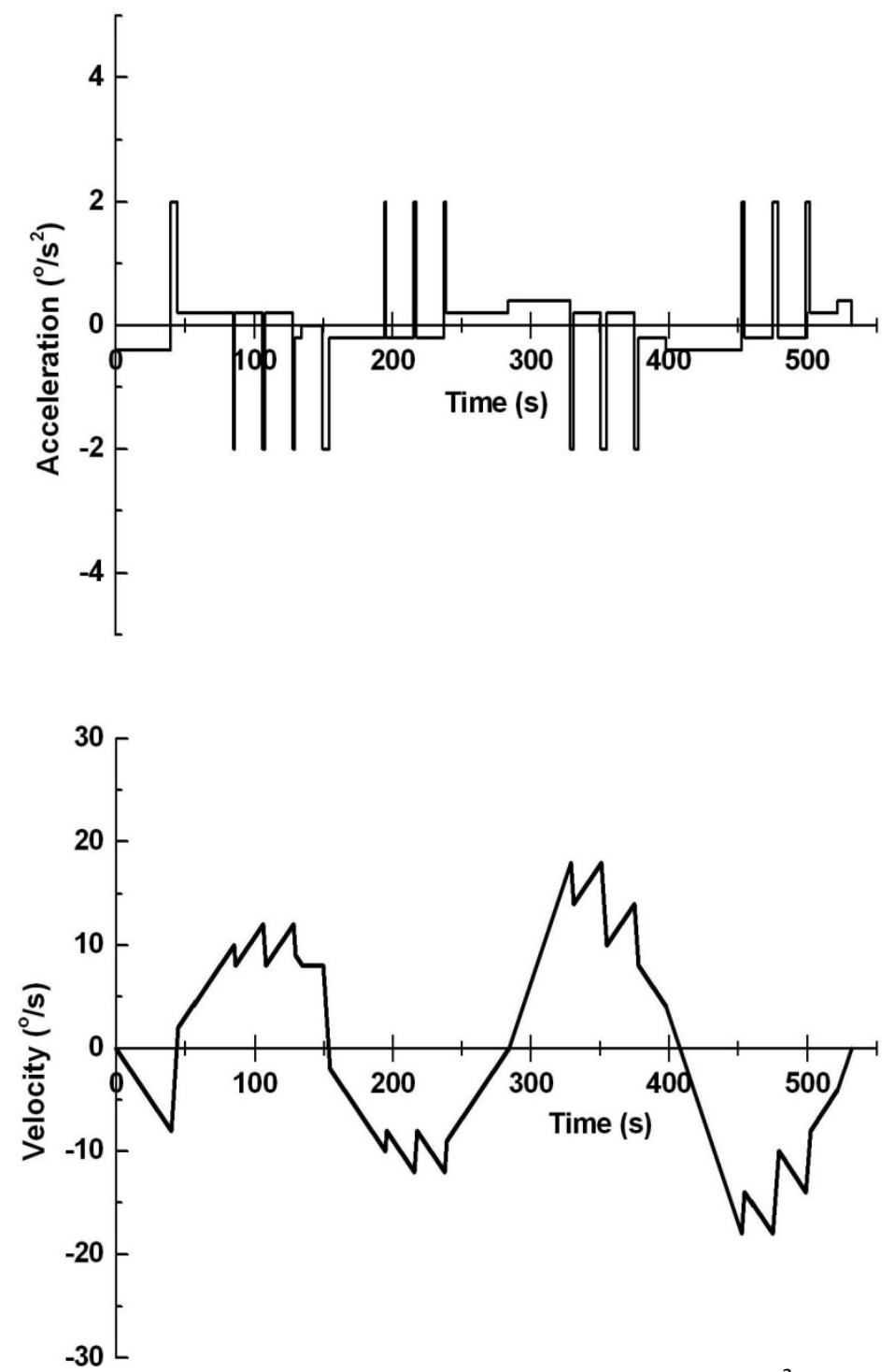

Figure C.2: Acceleration and velocity profile for healthy subjects with $2 \% \mathrm{~s}^{2}$ stimuli lasting between 1 and $5 \mathrm{~s}$, at least 20 s separated. Voice commands were given $5 \mathrm{~s}$ prior to a stimulus. Absolute velocity after a stimulus was at least $8 \%$ s. The profile was terminated after $284 \mathrm{~s}$ if a healthy subject had correctly perceived a 2,3 or $4 \%$ stimulus (with $0.2 \% \mathrm{~s}^{2}$ subthreshold accelerations between stimuli) to the left and right. If not, the profile was continued to the end (532 s) with 4, 6 and $8 \%$ s stimuli (with $0.4 \% \mathrm{~s}^{2}$ subthreshold accelerations between stimuli). Voice commands with no stimulus (catch trials) were given at 15 and $169 \mathrm{~s}$. See text for more details. 
After the clinical examination patients were seated in a chair (Nydiak 500, Ekida GmbH) in the light-tight room with a 3-points seatbelt, feet secured, each hand around a bar fixed to the armrest of the chair, eyes closed and masked, headphones mounted to mask auditory cues for spatial orientation and to communicate with the operator. To orient the horizontal canals with the plane of rotation (the earth-vertical axis), subjects flexed their neck slightly [von Brevern et al., 1997]. Patients were instructed to keep their head in this specific position, which was constantly monitored by the operator via an infra red sensitive camera. They underwent a clinical profile. After the clinical profile the head impulse test (HIT) on the horizontal canals was performed and recorded using a high speed video camera (Casio Exilim $300 \mathrm{fps}$ ) to determine a possible defective horizontal vestibular ocular reflex (VOR).

\section{Stimulus profile}

\section{Healthy subjects}

Subthreshold accelerations of 0.2 and $0.4 \% \mathrm{~s}^{2}$ were continuously applied and modulated with $2 \% \mathrm{~s}^{2}$ decelerations of short duration $(0-5 \mathrm{~s})$, resulting in stimuli between 0 and 10 $\%(0,2,3,4,6,8$ and $10 \%$ s). Based on a pilot study, it was established that the strongest stimuli $\left(2 \% \mathrm{~s}^{2}\right.$ for $5 \mathrm{~s}=10 \%$ ) were supra threshold. Inter-stimuli time was minimally $20 \mathrm{~s}$ and post-stimulus rotation velocity was minimally $8 \%$. The profile applied in healthy subjects is shown in figure C.2. Onset direction of the profile was randomized. Five seconds before the onset of each stimulus the operator warned the subject with a voice command. Additionally, 15 and 169 seconds after initiation of the profile, the subject was given a catch trial, i.e. a voice command, although 5 seconds later no stimulus $\left(0 \% \mathrm{~s}^{2}\right.$ deceleration, $0 \mathrm{~s}$ duration) was given.

The subjects were told beforehand that the chair would rotate constantly, to the right or left, but that they would not be able to feel this slow rotation. They were also informed that we would warn them about five seconds before a sudden small increase in the rotation velocity direction and ask them to verbally indicate the actual rotation direction (leftwards or rightwards) after the sudden increase of velocity. We told them that it was possible that sometimes no increase in rotation velocity could be perceived. If the subject did not respond within 5 seconds after the velocity change, the operator asked again if they felt any change in rotation. 

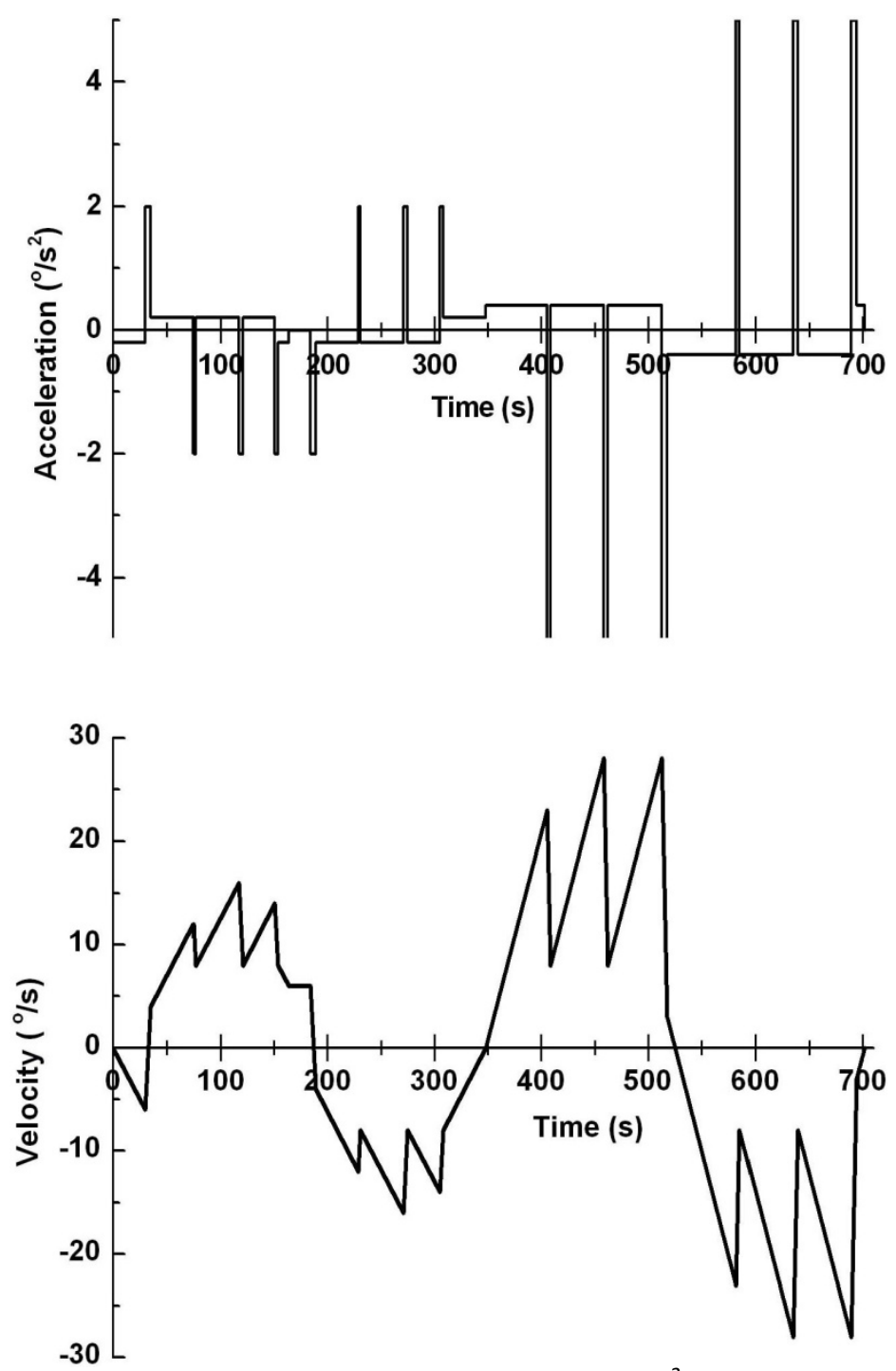

Figure C.3: Clinical acceleration and velocity profile with 2 or $5 \% \mathrm{~s}^{2}$ stimuli lasting between 2 and 5 $s$, at least $30 \mathrm{~s}$ separated. Voice commands were given $5 \mathrm{~s}$ prior to a stimulus. Absolute velocity after a stimulus was at least $8 \%$, except for the largest $25 \%$ stimulus. The profile was terminated after $348 \mathrm{~s}$ if a patient had correctly perceived a 4, 6, 8 or $10 \%$ stimulus (with $0.2^{\circ} / \mathrm{s}^{2}$ subthreshold accelerations between stimuli) to the left and right. If not, the profile was continued to the end (702 s) with 15, 20 and $25 \%$ stimuli (with $0.4 \% \mathrm{~s}^{2}$ subthreshold accelerations between stimuli). Voice commands with no stimulus (catch trials) were given at 10 and $164 \mathrm{~s}$. 
The profile was terminated after 284 seconds if the subject, during $0.2 \% \mathrm{~s}^{2}$ subthreshold accelerations, had correctly perceived a rotation deceleration stimulus of 2,3 or $4 \%$ to the left as well as to the right (all subjects except 1 perceived the supra threshold stimulus of $10 \%$ s). If not, the profile was continued with $0.4 \%$ accelerations until the end (532 s), which included stimuli of 4,6 and $8 \% \mathrm{~s}$ to the left and to the right. The threshold was defined as the smallest perceivable stimulus. 5 subjects were randomly selected and had 5 runs on the profile on 5 different days with the onset direction randomized. The remaining 12 subjects had only one run on the profile.

\section{Patients}

The patients underwent a clinical profile of short duration accelerations, based on the normative results obtained in the 17 healthy subjects. Subthreshold accelerations of 0.2 and $0.4 \% \mathrm{~s}^{2}$ were continuously applied and modulated with 2 or $5 \% \mathrm{~s}^{2}$ decelerations of short duration $(0-5 \mathrm{~s})$, resulting in stimuli between 0 and $25 \% \mathrm{~s}(0,4,6,8,10,15,20$ and $25 \%$ s). Inter-stimuli time was minimally $30 \mathrm{~s}$ and post-stimulus rotation velocity was minimally $8 \%$ s (except for the $25 \%$ stimulus). The clinical profile is shown in figure C.3. Onset direction of the profile was randomized. Five seconds before the onset of each stimulus the operator warned the patient with a voice command. 10 and 164 seconds after initiation of the profile, the subject was also given a catch trial, i.e. a voice command, although 5 seconds later no stimulus $\left(0 \% \mathrm{~s}^{2}\right.$ deceleration, $0 \mathrm{~s}$ duration) was given. The instructions were the same as for the healthy subjects.

The profile was terminated after 348 seconds if the subject had correctly perceived a rotation stimulus of $4,6,8$ or $10 \%$ to the left as well as to the right. If not, the profile was continued with $0.4 \% \mathrm{~s}^{2}$ subthreshold accelerations until the end (702 s), which included stimuli of 15,20 and $25 \%$ s. The threshold was defined as the smallest perceivable stimulus. 


\section{Data analysis}

Significant differences between left and right and between onset directions were determined using Wilcoxon's signed rank test. Significant departures from Gaussian distributions were identified using the Kolmogorov-Smirnov test. The level of significance in all tests applied was $p<0.05$. Average thresholds and $95 \%$ confidence intervals were determined for left and right stimuli. The $95 \%$ confidence interval was determined for the difference between left and right stimuli as well. Intra and interindividual variability was defined as the standard deviation relative to the average threshold, the former averaged over the 5 subjects selected for 5 runs.

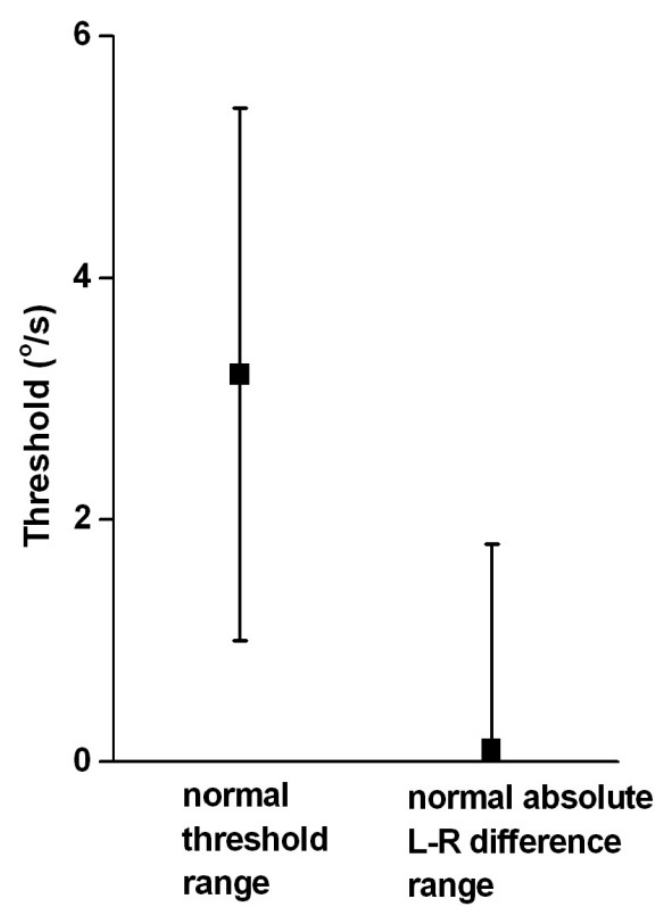

Figure C.4: Mean and 95\% confidence intervals for rotation perception thresholds (left or right) and for absolute left-right differences 


\subsection{Results}

\section{Healthy subjects}

One healthy male subject had a rotation perception threshold above the strongest stimulus $(10 \% \mathrm{~s})$. To exclude vestibular pathology, this subject underwent standard vestibular testing as described in the methods section, which were all normal. He also underwent the clinical profile and had a threshold that was even above the strongest stimulus in the clinical profile $(25 \% \mathrm{~s})$. This subject's data are not included in the remaining data analysis.

None of the healthy subjects reported sensation of rotation during subthreshold accelerations or visual, auditory or tactile cues about rotation direction. Subjects only had a rotation sensation during the stimuli above their individual thresholds, which they localized inside the head. None of them experienced any non-vestibular cues during the stimuli.

No gender effects were observed. The differences between left and right and between onset directions were not significant and not significantly different from a normal distribution. The average threshold $3.2 \%$ and left-right difference $0.1 \%$ and $95 \%$ confidence intervals are shown in figure C.4. The confidence interval of the threshold is from 1.0 to $5.5 \%$ ( $34 \%$ inter-individual variability) and between 0 and $1.8 \%$ for leftright differences. Because stimuli were given in steps of $2 \% \mathrm{~s}$, we specified the normal range up to $6 \%$ for left and right stimuli and up to $2 \%$ for left-right differences. No healthy subject had a threshold above $6 \%$, except the one mentioned above, and none had a left-right difference above $2 \%$.

During the 5 runs in the 5 selected subjects the threshold did not differ significantly between runs (Friedman test, $p>0.2$ ), indicating no adaptation or learning during subsequent runs. Intra-individual variability of the threshold was on average $17 \%$.

\section{Patients}

The patient results are shown in table C.1. Seven out of the 20 patients had an increased perception threshold for horizontal plane rotations to at least one side. The increased thresholds in these 7 patients confirmed the dominant horizontal canal paresis based on the caloric examination (threshold increased in 12 out of 14 ears), and especially the most severe pareses. Six out of these 7 patients also had an abnormal head impulse test 
(HIT). For the 10 patients (top 10 in table C.1, 20 ears) with unilateral or bilateral vestibular hypo- or areflexia (16 ears) the sensitivity of the perception threshold for horizontal plane rotations was 56 \% (9/16). For the 14 patients (top 14 in table C.1, 22 ears) with a horizontal canal paresis, the sensitivity of the perception test was $41 \%$ (9/22). Additionally, in 2 patients (no. 19 and 12) the perception threshold was increased to one side where the caloric examination showed no horizontal canal paresis.

Nine out of the 20 patients had an abnormal HIT to at least one side, which confirmed the dominant horizontal canal paresis (HIT abnormal in 13 out of 18 ears). For the 10 patients with unilateral or bilateral vestibular hypo- or areflexia the sensitivity of the HIT was $75 \%(12 / 16)$. For the 14 patients with a horizontal canal paresis, the sensitivity of the HIT was $59 \%(13 / 22)$. There was no patient who had an increased perception threshold where the caloric examination showed no horizontal canal paresis.

McNemar's test showed no significant difference $(p>0.2)$ in sensitivity between the HIT and perception threshold for horizontal plane rotations.

\subsection{Discussion}

\section{Healthy subjects}

In this study deceleration step stimuli of short duration opposite to the actual rotation direction were applied during continuous subthreshold acceleration rotation to induce vestibular rotation perception. Rotation perception is a multi sensory task [Merfeld et al., 2005a, Merfeld et al., 2005b] which complicates the detection of the vestibular thresholds of rotation perception. However, we succeeded in masking the non vestibular (visual, auditory or tactile) directional information effectively during the applied rotation profiles, because our healthy subjects and patients reported no other cues than a vestibular rotational sensation during the stimuli above their individual thresholds. All subjects indicated the direction of the stimuli and not the direction of the chair rotation.

The average threshold and confidence interval $(3.2 \%$ and 1.0 to $5.5 \%$ s) of perception of horizontal plane rotations found in this study were not significantly different $(p>0.05$ using the two-sample t-test for independent samples with unequal variances) from the thresholds found by Grabherr et al. [Grabherr et al., 2008] at 0.05 and $0.1 \mathrm{~Hz}(0.4$ to 5.6 and 1.1 to $4.1 \%$ respectively), who used single cycles of sinusoidal accelerations. Our 
confidence interval is significantly $(p<0.05)$ higher than found at frequencies of $0.2 \mathrm{~Hz}$ $(0.2$ to $3.4 \%$ s) and above [Grabherr et al., 2008], again indicating no non vestibular high frequent parts in our stimuli. Our confidence interval was also not significantly different from the data by Clark and Stewart $\left(0.17-0.87 \% \mathrm{~s}^{2}\right.$ using $10 \mathrm{~s}$ stimuli) [Clark and Stewart, 1970], thus opening the possibility for a clinical test with this new motion profile paradigm on rotation perception.

\section{Patients}

Our patient population was composed of patients with no function loss of the horizontal canals up to bilateral vestibular areflexia; a wide range in terms of function losses. The perception thresholds for horizontal plane rotations were increased in especially those patients with the most severe pareses based on the classical tests. Three out of our 20 patients had a bilateral vestibular areflexia and had increased thresholds to both sides, in line with Metcalfe and Gresty [Metcalfe and Gresty, 1992].

The age from our patients was significantly higher than from our healthy subjects, but we assumed no effect of age because Seemungal et al. [Seemungal et al., 2004] showed no effect of age on the threshold of yaw rotation perception.

The slow component eye velocity during a torsion swing and the perception of rotation induced by deceleration step stimuli of short duration during continuous (subthreshold acceleration) rotation both are a test of bilateral horizontal semicircular canal functionality. We expected this rotation perception test to be more sensitive than head impulse testing (HIT), because of its analogy to perception tests for hearing (audiometry) and vision (visual acuity testing) and because it might have a closer correlation to patients' complaints about vertigo [Kanayama 1995]. In 3 (with bilateral vestibular areflexia) out of the 18 patients the torsion swing test was abnormal and 7 out of the 20 patients had an increased rotation perception threshold to at least one side. McNemar's test however showed no significant difference $(p=0.06)$ in sensitivity between the torsion swing and perception threshold for horizontal plane rotations.

The frequency content of the stimuli applied differs in the caloric test, head impuls test, torsion swing test, and the perception tests, which might lead to differences in outcome. Additionally, HIT is a test for unilateral canal functionality [Halmagyi and Curthoys, 1988], like the caloric test [Schmid-Priscoveanu et al., 2001], whereas the torsion swing and rotation perception test are tests for bilateral canal functionality. 
The rotation perception test as described in this study is however not more sensitive than the HIT and both still have a limited sensitivity of about $40-60 \%$.

\subsection{Conclusion}

The vestibular rotation perception test - deceleration step stimuli of short duration opposite to the actual rotation direction during continuous subthreshold acceleration rotation - is easy to perform and reproducible in healthy subjects, making it a relatively simple clinically applicable quantitative test. The reports of healthy subjects and patients indicated that we were able to mask non-vestibular cues of rotation.

Interestingly, we showed that 13 out of 18 patients with a partial or complete unilateral or bilateral caloric hypofunction have normal rotation perception thresholds. It might be that in some unilateral affected patients, 1) the non affected side is responsible for the normal thresholds. Otherwise it might be that 2) their vertical canals and otolith function enable sufficient rotation perception in the horizontal plane, or that 3 ) the vestibular losses might have specific frequency dependence outside the frequency range tested. Also Metcalfe and Gresty [Metcalfe and Gresty, 1992] and von Brevern et al. [von Brevern et al., 1997] have shown that a significant part of patients with chronicstage unilateral nerve section, i.e. total function loss of otolith and canal function on one side, show normal responses for horizontal canal rotation perception testing. In patients with the most severe bilateral horizontal canal function losses, also rotation perception thresholds were increased. Together with differences in the frequency content of caloric, torsion swing, head impulse and the perception threshold detection presented here, this indicates that rotation perception as regular clinical tool might have a complementary value, but does not replace these tests.

Using the oculogyral illusion as a perception measure might be even more sensitive than the protocol applied in this study, because a significantly lower threshold has been reported using this illusion [Benson et al., 1989]. 


\section{CHAPTER 5: THRESHOLDS OF TILT AND TRANSLATION PERCEPTION IN HEALTHY SUBJECTS}

\subsection{Introduction}

Perception of body tilt is accomplished by multisensory input [Bringoux et al., 2003, Bronstein, 1999], in particular from the vestibular organs (otolith organs and semicircular canals), vision and the somatosensory system (including propriocepsis, interoceptors and touch [Bringoux et al., 2003]). It has mainly been investigated by adjusting a visual or haptic indicator [Clement and Deguine, 2010, Vingerhoets et al., 2009, Seidman et al., 2009, Guenther et al., 2009, Mazibrada et al., 2008, Wright and Horak, 2007, Lopez et al., 2007, Ceyte et al., 2007, Tribukait and Eiken, 2006, Trousselard et al., 2003, Jarchow and Mast, 1999, Anastasopoulos et al., 1999, Anastasopoulos et al., 1997, Ito and Gresty, 1996, Raphel and Barraud, 1994, Ross et al., 1969]. Indicator adjustment is known to be based on centrally weighted fusion of visual, vestibular and egocentric references [Vingerhoets et al., 2009]. This enables a healthy person in upright position to very accurately ${ }^{2}$ (standard deviation $0.5^{\circ}$ ) align an indicator with the direction of gravity [Trousselard et al., 2003]. This integration of available sensory information is an adaptive process referred to as 'sensory re-weighting' [Mahboobin et al., 2009].

Other methods to investigate human tilt perception are perception of body orientation [Mazibrada et al., 2008, Bringoux et al., 2003, Bringoux et al., 2002, Teasdale et al., 1999, Bisdorff et al., 1996, Fitzpatrick and McCloskey, 1994] and controlling a device to which a subject is restrained [Jarchow and Mast, 1999, Aoki et al., 1999, Anastasopoulos et al., 1999, Anastasopoulos et al., 1997, Ito and Gresty, 1996, Riccio et al., 1992, Nelson, 1968].

By excluding vision and canal stimulation by induction of slow rotations, perception is based on otolithic and somatosensory information only. Since already very small head tilts lead to modulation of the otolith afferents [Fernandez and Goldberg, 1976], it has for long been suggested that the otolith system is the primary sensor system for body tilt perception and postural control [Mittelstaedt, 1999]. Also, based on its physical properties, the otolith system might have the highest sensitivity for low frequency tilts

\footnotetext{
${ }^{2}$ The term accuracy is used to indicate bias or the proximity of a result to the true value. The term precision is used to indicate variable errors, uncertainty or repeatability.
} 
(gravity and constant linear accelerations $<1 \mathrm{~Hz}$ ) compared to all other sensory systems [Melvill Jones, 1979]. For this reason most studies on body tilt perception have searched for threshold properties of the otolith system.

A dominant role of the somatosensory system for the perception of body uprightness has however emerged [Yi and Park, 2009, Vaugoyeau et al., 2008, Mazibrada et al., 2008, Bringoux et al., 2003, Bringoux et al., 2002, Teasdale et al., 1999, Bronstein, 1999, Aoki et al., 1999, Anastasopoulos et al., 1999, Ito and Gresty, 1996, Bisdorff et al., 1996, Fitzpatrick and McCloskey, 1994, Mittelstaedt and Mittelstaedt, 1997, Vaitl et al., 2002] and especially when somatosensory information is masked or distorted, body orientation perception is neither accurate nor precise [Bringoux et al., 2003, Aoki et al., 1999, Nelson, 1968]. Nevertheless, precision of the perceived vertical in quasi-static conditions is improved with vestibular input [Bisdorff et al., 1996, Nelson, 1968, Clark and Graybiel, 1963] and improved with somatosensory input [Bringoux et al., 2003, Teasdale et al., 1999, Riccio et al., 1992, Ross et al., 1969]. Furthermore it has been shown that during perturbations of posture somatosensory and vestibular inputs are dynamically integrated [Tribukait and Eiken, 2006, Aoki et al., 1999], indicating that both the otolith and somatosensory system are important for precision of body orientation perception.

But even when vestibular and somatosensory information are present, accuracy of perception of body orientation is very poor [Bringoux et al., 2003, Ito and Gresty, 1996] and deteriorates due to tilt adaptation [Bringoux et al., 2003, Ito and Gresty, 1996, Bisdorff et al., 1996, Clark and Graybiel, 1964, Ross et al., 1969], which occurs rapidly within a minute [Bisdorff et al., 1996]. Additionally, it was recently suggested [Nooij, 2008] that the vestibular organs play an important role in the detection of orientation relative to the vertical at relatively small tilt angles, whereas larger tilt angle are predominantly detected with somatosensory and proprioceptive cues, be it with a limited accuracy. Moreover, the accuracy of body perception has been shown not to be different between normals and bilateral somatosensory deafferented [Mazibrada et al., 2008] or vestibular patients [Bringoux et al., 2002].

To study a possible otolithic threshold for body tilt perception, it is evident that exclusion of auditory, visual and semicircular canal information is essential [Merfeld et al., 2005b] but very difficult. Moreover, the otolithic threshold might be higher than the somatosensory threshold [Fitzpatrick and McCloskey, 1994] and accuracy of perception 
of body orientation is very poor [Bringoux et al., 2003, Ito and Gresty, 1996]. Therefore investigation of precision - changes in the direction (and/or magnitude) of linear acceleration - is essential, and not accuracy - the absolute direction -, to find an otolithic threshold [Nelson, 1968]. This is however in practice very difficult as tactile and semicircular canal cues need to be avoided. Perception of a rotating linear acceleration vector during off-vertical axis rotation (OVAR) is for example known to be largely influenced by the somatosensory system [Holly et al., 2009, Benson et al., 1975].

Reviewing the literature on body orientation perception, the threshold for body tilt perception seems to have a substantial inter-individual range for healthy subjects and appears to be about minimal $2^{\circ}$ :

- The group of Christian Raphel [Bringoux et al., 2003, Bringoux et al., 2002, Teasdale et al., 1999] instructed subjects to indicate verbally when they perceived a change in body orientation due to slow pitch body tilts $(0.05 \% \mathrm{~s})$ in total darkness, starting from an initial vertical body orientation while standing against an L-shaped vertical platform. Without body fixation they observed thresholds of about $1.5^{\circ} \pm 0.5^{\circ}$ [Teasdale et al., 1999], a situation dominated by somatosensory input. While strapping or completely immobilizing the subjects in a body cast, the threshold was $16^{\circ} \pm 2^{\circ}$ in the body cast condition and $8^{\circ} \pm 2^{\circ}$ in the strapped condition [Bringoux et al., 2003]. However, since platform tilt velocity was so slow that it took 1 minute to tilt $3^{\circ}$, their results might have been negatively influenced by adaptation to prolonged tilt. Especially because they observed no significant difference between healthy subjects and bilateral vestibular areflexia patients [Bringoux et al., 2002] while seated and immobilized (threshold of $5^{\circ} \pm 2^{\circ}$ in roll, $6^{\circ} \pm 2^{\circ}$ in pitch).

- Adolfo Bronstein's group [Bisdorff et al., 1996] used a similar approach, while subjects were seated in a padded chair, for pitch and roll body tilts $\left(1.5^{\circ} / \mathrm{s}\right)$ in total darkness. Tilt was perceived at $6^{\circ} \pm 2^{\circ}$ for both pitch and rolls in normal subjects and was significantly higher in bilateral vestibular areflexia patients.

The literature on controlling a device to which a subject is restrained [Jarchow and Mast, 1999, Aoki et al., 1999, Anastasopoulos et al., 1999, Anastasopoulos et al., 1997, Ito and Gresty, 1996, Riccio et al., 1992, Nelson, 1968] to a horizontal or vertical orientation is 
not further discussed in this manuscript. All these studies primarily focused on the accuracy of subjects, which seems to be dominated by the somatosensory system.

The otolith organs respond equivalently to changes in gravitational force due to head tilt and to changes in inertial force due to linear acceleration [Zupan and Merfeld, 2008, Groen and Bles, 2004]. Because of this ambiguity of the otolith system for translation and tilt, motion direction perception during whole body linear accelerations in darkness has also been used to determine the threshold for body tilt perception [Zupan and Merfeld, 2008, Kingma, 2005, Gianna et al., 1996, Benson et al., 1986, Melvill Jones and Young, 1978]. However, acceleration thresholds vary with the stimulus profile used to determine the threshold (sinusoidal, parabolic, linear, stepwise), and thresholds expressed in terms of velocity are more constant and less variable [Kingma, 2005]. This is due to the response characteristics of the otoliths: after a step in linear acceleration the macula deflection is exponential with a time constant of about 1 second [Melvill Jones, 1979, Guedry, 1974], which can initially be approximated with linear deflection after initiation of the acceleration step. This results in an inverse relationship between the duration and detection threshold for constant linear accelerations [Melvill Jones and Young, 1978]. This suggests to use the product of the acceleration and its duration to quantify the threshold for short duration stimuli. This product is similar to the Mulder product for perception of angular accelerations [Clark et al., 1980, Melvill Jones and Young, 1978].

- Melvill Jones and Young [Melvill Jones and Young, 1978] found the linear velocity threshold to be at least $15 \mathrm{~cm} / \mathrm{s}$ based on response latencies to acceleration steps superimposed on linear baseline velocities

- Gianna et al. [Gianna et al., 1996] used steps, linear and parabolic transients in linear accelerations and found the threshold to be at least $5 \mathrm{~cm} / \mathrm{s}$. Their stimuli however started from zero velocity, which not only stimulates the otolith organs, but also gives tactile cues

- Others [Kingma, 2005, Zupan and Merfeld, 2008, Benson et al., 1986] also found the threshold to be at least $5 \mathrm{~cm} / \mathrm{s}$ during single cycle sinusoidal stimuli, which however contains a certain periodicity which is by nature predictable and might lower the threshold [Kingma, 2005, Benson et al., 1986] 
So reviewing the literature on translation perception, we conclude that the threshold for motion direction perception has a substantial inter-individual range for healthy subjects and appears to be about $15 \mathrm{~cm} / \mathrm{s}\left(5 \mathrm{~cm} / \mathrm{s}^{2}\right.$ ( $0.3^{\circ}$ of tilt) for $3 \mathrm{~s}$ or $15 \mathrm{~cm} / \mathrm{s}^{2}$ ( $\sim .9^{\circ}$ of tilt) for $1 \mathrm{~s})$ which is significantly lower than the threshold for body tilt perception, possibly because of somatosensory cues.

The goal of this study was to determine the perception ${ }^{3}$ threshold for various stimuli. More specifically, we investigated in 3 new motion/tilt profile paradigms the extent to which subjects can perceive

- tilt sensations during unilateral and bilateral eccentric rotations [Merfeld et al., 2005a] while seated, which appears to be a promising tool for the evaluation of vestibular (utricular) dysfunction [Clement and Deguine, 2010]. Especially because the effect mainly depends on vestibular and not on somatosensory function

- whole body left-right and fore-aft decelerations opposite to the actual linear velocity direction [Melvill Jones and Young, 1978] while seated. In this way the impact of somatosensory cues were reduced and specific vestibular translation/tilt perceptions were induced

- tilt sensations during discrete slow velocity platform roll and pitch tilts while seated

3 The term perception is here defined as the ability to give a subjective report about his motion/tilt [Fitzpatrick and McCloskey, 1994]. 


\subsection{Methods}

15 healthy subjects (12 male, 3 female) without any history or evidence of any ophthalmologic or neuro-otological disorder participated in this study. Ages ranged from 18-31 years, mean 23 years. All subjects participated on a voluntary basis after giving their informed consent. 3 subjects were randomly selected and had 5 runs on all profiles (on 5 different days). The remaining 12 subjects had only one run on the profiles.

\section{Centrifugation}

Whole body eccentric rotations were generated by a rotational chair (Jaeger-Toennies) equipped with a motor driven linear sled to translate the chair perpendicular to the rotational axis [Kingma, 2006]. To achieve unilateral eccentricity, subjects were gradually translated $3.75 \mathrm{~cm}$ after having reached maximum rotational velocity [Nowe et al.], so that one utricule is on the rotation axis and the other is centrifugated at $7.5 \mathrm{~cm}$ [Clarke et al., 2003]. To achieve bilateral eccentricity, subjects were first translated $50 \mathrm{~cm}$, after which the chair slowly accelerated to a maximum velocity. In both conditions a centrifugal acceleration is delivered, perpendicular to gravity, resulting in a tilted linear acceleration vector (gravito inertial acceleration).

Subjects, were seated upright in the chair with their neck slightly flexed, tightly restrained with a 4-points seatbelt (to avoid potential tactile cues induced by movement of the subject relative to the chair), with their feet on a footrest and lower legs tightly restrained, head fixed with a restraining headband and vacuum pillow, eyes closed and masked, each hand around a bar fixed to the armrest of the chair, and headphones to mask auditory cues for spatial orientation and to communicate with the operator. To further minimize possible visual cues, the chair was rotated in a light-tight room about an earth-vertical (yaw) axis.

\section{Stimulation protocol}

- Unilateral centrifugation; the chair was accelerated on centre at $2 \% \mathrm{~s}^{2}$ up to an angular velocity of $360 \%$ s. On centre rotation was maintained for $30 \mathrm{~s}$ to ensure that the semicircular canal response had decayed. The chair was then translated radial to the right and left to a maximum eccentricity of $3.75 \mathrm{~cm}$ at 3 different velocities $(0.5,1.5$ and $3 \mathrm{~mm} / \mathrm{s}$ with a rise time in the order of $0.1 \mathrm{~s})$. Maximal eccentricity was maintained for $30 \mathrm{~s}$, where after the chair was translated back 
to its on centre position. Translation direction and velocities (from and toward on centre) were randomized, resulting in 12 different stimuli. An example is shown in figure C.5. $30 \mathrm{~s}$ after the last radial chair translation back to the on centre position, the chair was decelerated with $2 \% \mathrm{~s}^{2}$. Total profile time was 20 $\min$.

Subjects were told that the chair would constantly rotate very rapidly during the stimuli, but that they would only slightly feel the rotation at the beginning and end of the profile. They were informed that we would warn them about five seconds before initiation of each chair translation and asked them to verbally indicate as soon as possible the perceived tilt direction (left- or rightwards). Because the slowest chair translation took $75 \mathrm{~s}(0.5 \mathrm{~mm} / \mathrm{s}$ toward $3.75 \mathrm{~cm})$ subjects were asked at 25 and $50 \mathrm{~s}$, if they had not responded yet, if they had felt any change in orientation. Failure to respond during translation was recorded as an unperceived movement (which never occurred). Likewise, a response in the direction opposite to the actual stimulus was recorded as an unperceived movement (which never occurred). Per subject and per stimulus the moment at which tilt direction was perceived was recorded and converted to the angle at which tilt direction was perceived using this equation:

$$
\underline{\left(\tan ^{-1}\left[\frac{\omega^{2} \cdot\left\{r-r_{0}\right\}}{G}\right]+\tan ^{-1}\left[\frac{\omega^{2} \cdot\left\{r+r_{0}\right\}}{G}\right]\right)}
$$

with $\omega(\mathrm{rad} / \mathrm{s})$ the angular velocity, $r$ the eccentricity $(\mathrm{m}), \mathrm{r}_{0}$ the utricular distance to the center of the head $(3.75 \mathrm{~cm}$ [Nowe et al., 2003]) and $\mathrm{G}$ the gravitational acceleration $\left(9.81 \mathrm{~m} / \mathrm{s}^{2}\right)$, resulting in a maximum tilt angle of $8.4^{\circ}$ at $3.75 \mathrm{~cm}$ eccentricity. Thus, the 3 different eccenter velocities equal $0.11,0.34$ and $0.67 \%$ tilt velocities. We do realize however that interpretation of this tilt angle is difficult, because one utricle is centrifugated and the other is on the rotation axis. This results in different signals from the left and right utricle, which might centrally be processed different (inhibited?) than equivalent head or body tilts [Kingma et al., 2001]. 
- Bilateral centrifugation; the chair was translated $50 \mathrm{~cm}$ to the right, and subthreshold accelerated backward at $0.4 \% \mathrm{~s}^{2}$ up to $97.5 \%$. This velocity was maintained for $30 \mathrm{~s}$, where after the chair decelerated at $0.4 \% \mathrm{~s}^{2}$. Total profile time was $8.5 \mathrm{~min}$.

Subjects were told that the chair would constantly rotate backward, but that they would not be able to feel that slow rotation. They were asked to verbally indicate tilt direction as soon as they perceived any tilt (rightward during acceleration and leftward during deceleration). Subjects were asked every $30 \mathrm{~s}$ if they had felt any tilt and its direction. Per subject and per stimulus the moment at which tilt direction was perceived was recorded and was converted to the angle at which tilt direction was perceived, also using equation 1 , which was maximally $8.4^{\circ}$ at $97.5^{\circ} / \mathrm{s}$. Tilt velocity was on average $0.034 \%$.

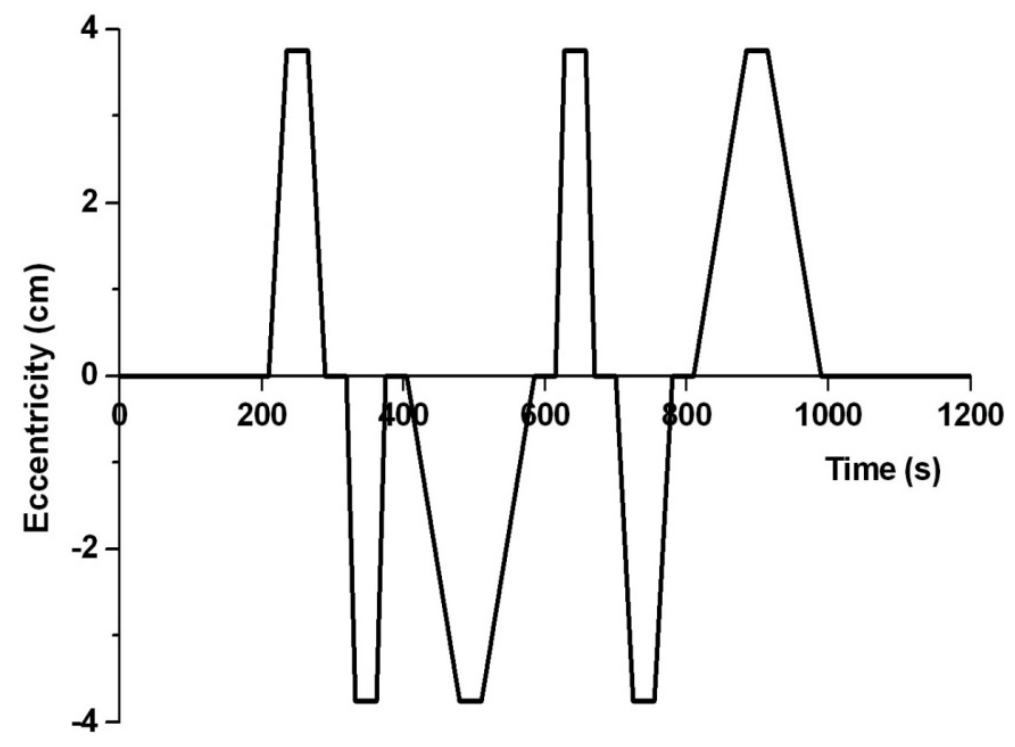

Figure C.5: Example of 12 stimuli during an eccenter profile for unilateral centrifugation during $360 \%$ rotation. Voice commands were given 5 s prior to a stimulus. 


\section{Linear sled}

Whole body motions while seated were generated by a motor driven linear sled (Maastricht Instruments BV) running on an earth-horizontal track of 4.2 meters [Kingma, 2005]. The seat could be changed into one of two positions in which the a. naso-occipital (AP) or b. interaural (LR) axis of the head was parallel to the direction of motion, resulting in an effective length of the track of a. $2.32 \mathrm{~m}$ and b. $3.04 \mathrm{~m}$. The motor was controlled by an embedded computer, which moved the sled according to a preprogrammed profile with a $0.01 \mathrm{~mm}$ resolution at a $1 \mathrm{kHz}$ sample rate.

Subjects, asked to wear clothes with long sleeves and were seated upright in the chair, tightly restrained with a 4-points seatbelt, to avoid potential tactile cues induced by movement of the subject relative to the chair. Their feet were on a foam footrest to avoid potential tactile cues from the sled. The head was fixed with a restraining headband to avoid potential accessory cues from the canals due to pitching or rolling angular movements of the head potentially induced by the horizontal linear accelerations [Melvill Jones and Young]. Eyes were closed and masked, hands folded between the legs, and headphones with prerecorded sled motor sound played back at $+50 \%$ speed in natural tones (Digital Pitch Control) using an IC Recorder (ICD-UX71) to mask auditory cues for spatial orientation and to communicate with the operator. To further minimize possible visual cues, the sled was moved in a light-tight room, and to further minimize the impact of wind on movement perception, the head was covered with a cloth. Upon request, all subjects indicated that they had no cues for detection of sled motion, except for the given stimuli, which opposed the movement direction of the sled to minimize possible cues about motion, direction and velocity.

\section{Stimulation protocol}

A subthreshold sled acceleration of $2 \mathrm{~cm} / \mathrm{s}^{2}$ (based on Melvill Jones and Young [Melvill Jones and Young, 1978] and a pilot study in 10 healthy subjects) was used to reach the required sled velocity (track length limited the maximum velocity to a. naso-occipital 25 $\mathrm{cm} / \mathrm{s}$ and $\mathrm{b}$. interaural $30 \mathrm{~cm} / \mathrm{s}$ ), after which the sled continued its motion at a constant velocity. After three or more seconds at this constant velocity, decelerations $(0,5,10$ and $20 \mathrm{~cm} / \mathrm{s}^{2}$ ) of short duration $(0-4 \mathrm{~s})$ were given, resulting in a total of a. eighteen $0-$ $20 \mathrm{~cm} / \mathrm{s}$ and b. twenty $0-25 \mathrm{~cm} / \mathrm{s}$ stimuli in steps of $5 \mathrm{~cm} / \mathrm{s}$, shown in table C.2. Stimuli were separated at least $15 \mathrm{~s}$ and the velocity after a stimulus was at least $5 \mathrm{~cm} / \mathrm{s}$. Each acceleration step was smoothed with a cosine shape of $0.5 \mathrm{~s}$ to avoid jerks, and was 
implemented in such a way that the net acceleration equaled the original step. Total profile time was a. 8 min., shown in figure C. 6 and b. 10.5 min., shown in figure C.7.
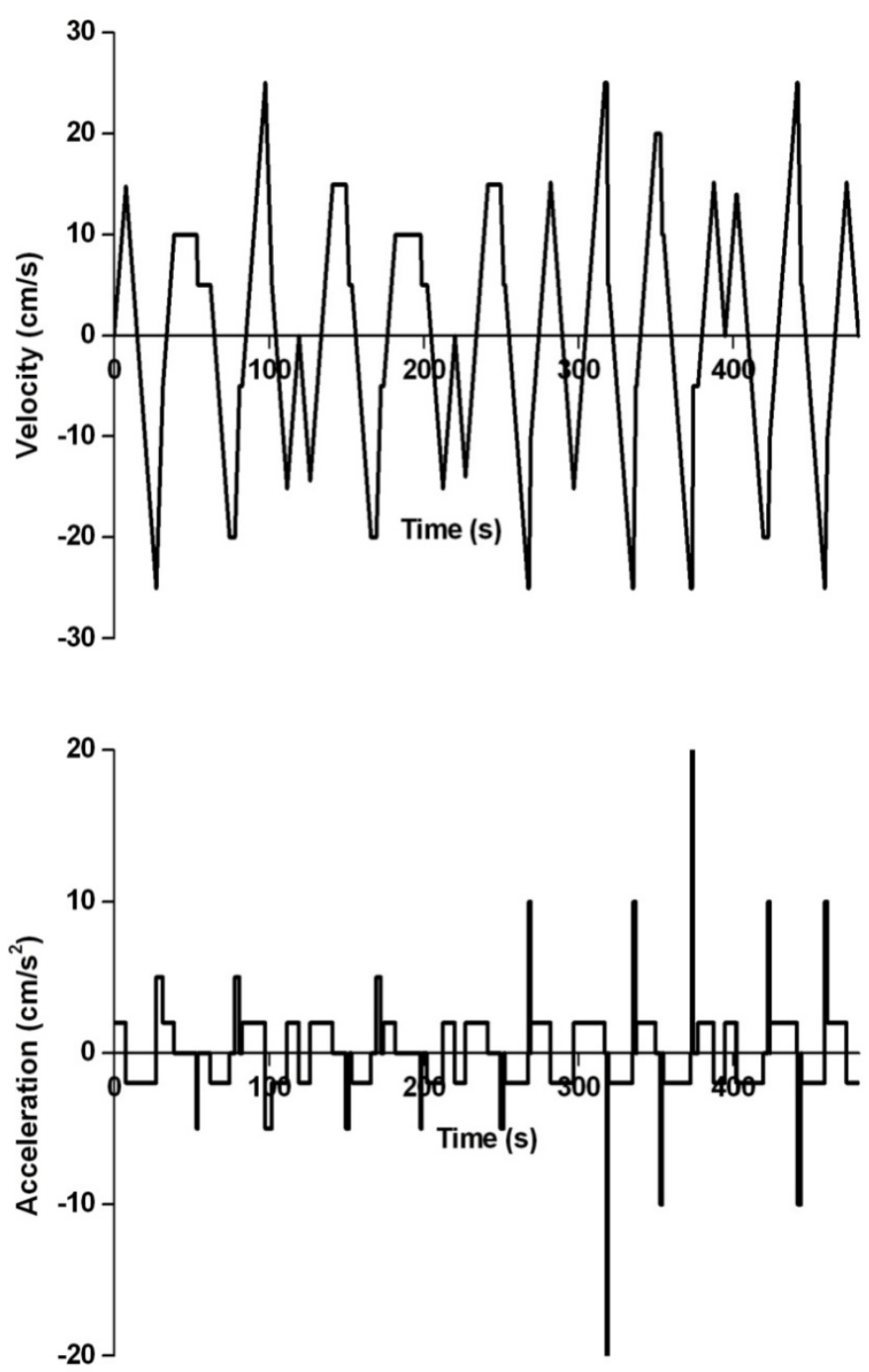

Figure C.6: Naso-occipital velocity and acceleration profile on the linear sled with 5, 10 and 20 $\mathrm{cm} / \mathrm{s}^{2}$ stimuli of 1-4 s, at least $15 \mathrm{~s}$ separated. Voice commands were given $5 \mathrm{~s}$ prior to each stimulus. Velocity after a stimulus was at least $5 \mathrm{~cm} / \mathrm{s}$. Voice commands with no stimulus were given at 193 and $214 \mathrm{~s}$. 
Table C.2: The a. 18 naso-occipital and b. 20 interaural stimuli given on the linear sled.

\begin{tabular}{llll}
$\begin{array}{l}\text { Stimuli } \\
(\mathbf{c m} / \mathrm{s})\end{array}$ & $\begin{array}{l}\text { combinations } \\
\text { time }(\mathbf{s}) \times \text { acceleration }\left(\mathrm{cm} / \mathrm{s}^{2}\right)\end{array}$ & \#per direction & seat orientation \\
\hline 0 & $0 \times 0$ & 2 & $\mathrm{a}+\mathrm{b}$ \\
5 & $1 \times 5$ & 2 & $\mathrm{a}+\mathrm{b}$ \\
10 & $1 \times 10,2 \times 5$ & 4 & $\mathrm{a}+\mathrm{b}$ \\
15 & $1.5 \times 10,3 \times 5$ & 4 & $\mathrm{a}+\mathrm{b}$ \\
20 & $1 \times 20,2 \times 10,4 \times 5$ & 6 & $\mathrm{a}+\mathrm{b}$ \\
25 & $2.5 \times 10$ & 2 & $\mathrm{~b}$
\end{tabular}

The velocity and acceleration profile for healthy subjects are shown in figures 6 (AP) and 7 (LR). Five seconds before the onset of each stimulus the operator warned the subject with a voice command. a. 193 and 214 (AP profile) and b. 212 and 244 (LR profile) seconds after initiation of the profile, the subject was also given a voice command, although 5 seconds later no stimulus $\left(0 \mathrm{~cm} / \mathrm{s}^{2}\right.$ deceleration, 0 s duration) was given.

The subjects were told that the chair would move constantly, to the a. front or back or $b$. right or left, but that they would not be able to feel that slow movement. They were also informed that we would warn them about five seconds before a sudden small increase in the motion velocity direction and asked them to verbally indicate the actual motion direction of the chair (a. for- or backwards or b. left- or rightwards) after the sudden increase of velocity. They first practiced on an introduction profile with $30-40 \mathrm{~cm} / \mathrm{s}$ stimuli, where after they were given the AP and LR profile. We then told them that in the actual profile it was possible that sometimes no increase in motion velocity could be perceived. If the subject did not respond within 5 seconds after the velocity change, the operator asked again if they had felt any change in motion. Failure to respond was recorded as an unperceived movement (which occurred for all $0 \mathrm{~cm} / \mathrm{s}$ stimuli). Likewise, a response in the direction opposite to the actual stimulus was recorded as an unperceived movement [Fitzpatrick and McCloskey, Melvill Jones and Young, 1978]. Per subject and per stimulus the proportion of correct detection was calculated [Gianna et al., 1996]. 

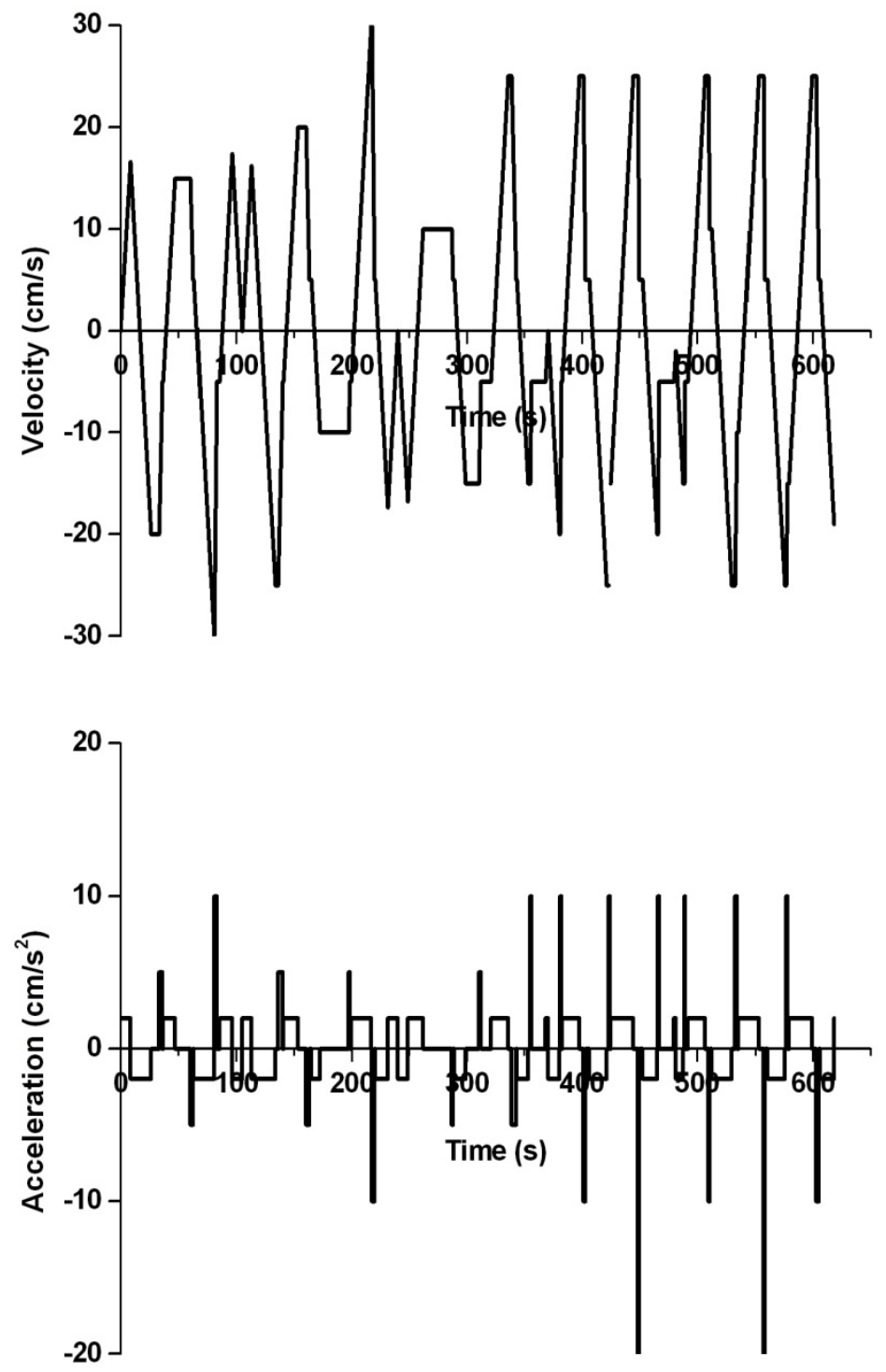

Figure C.7: Interaural velocity and acceleration profile on the linear sled with 5,10 and $20 \mathrm{~cm} / \mathrm{s}^{2}$ stimuli of 1-4 s, at least $15 \mathrm{~s}$ separated. Voice commands were given $5 \mathrm{~s}$ prior to each stimulus. Velocity after a stimulus was at least $5 \mathrm{~cm} / \mathrm{s}$. Voice commands with no stimulus were given at 212 and $244 \mathrm{~s}$. 


\section{Tilt platform}

Whole body tilts while seated were generated by a tilting platform (Maastricht Instruments BV) in roll and pitch direction randomly. The platform was controlled by an embedded computer, which tilted the platform to pre-programmed tilt orientations with a $0.01^{\circ}$ resolution and $1 \mathrm{kHz}$ sample rate with a tilt velocity of $0.1 \%$ (rise time of about $0.1 \mathrm{~s})$.

Subjects were seated [Bringoux et al., 2002, Bisdorff et al., 1996] on a chair positioned on the platform without restraints, with their feet on a foam footrest (to avoid potential tactile cues from the platform), eyes closed and masked, hands on the knees, and headphones to mask auditory cues for spatial orientation and to communicate with the operator. To further minimize possible visual cues, the platform was tilted in a light-tight room. Upon request, all subjects indicated that they had no cues for absolute platform orientation as soon as the platform started moving.

\section{Stimulation protocol}

A sub canal threshold platform tilt velocity of $0.1 \%$ was used to reach the preprogrammed tilt orientations, $0.5,1.0,1.5,2.0$ and $3.0^{\circ}$ left-, right-, for- and backward, from and towards absolute horizontal platform orientation, resulting in a total of 40 stimuli. After the subject's response, the operator pushed a button to move the platform to the next orientation. Total profile time was on average $14 \mathrm{~min}$. The platform orientation profile is shown in figure C.8.

The subjects were told that the platform would tilt left-, right-, for- and backward independently, and would not tilt for example left- and forward at the same time. They were also informed that the operator would warn them just before each platform orientation change with a voice command and asked them to verbally indicate the actual tilt direction of the platform. Subjects were instructed to use all available body sensations and verbally indicate as soon as they felt the direction of tilt [Bringoux et al., 2003]. We then told them that it was possible that sometimes no orientation change could be perceived. If the subject did not respond during or directly after an orientation had been reached, the operator asked again if they had felt any change in orientation. Failure to respond was recorded as an unperceived movement. Likewise, a response in a direction other than the actual tilt direction was recorded as an unperceived movement 
[Fitzpatrick and McCloskey, Melvill Jones and Young, 1978]. Per subject and per stimulus the proportion of correct detection was calculated [Gianna et al., 1996].

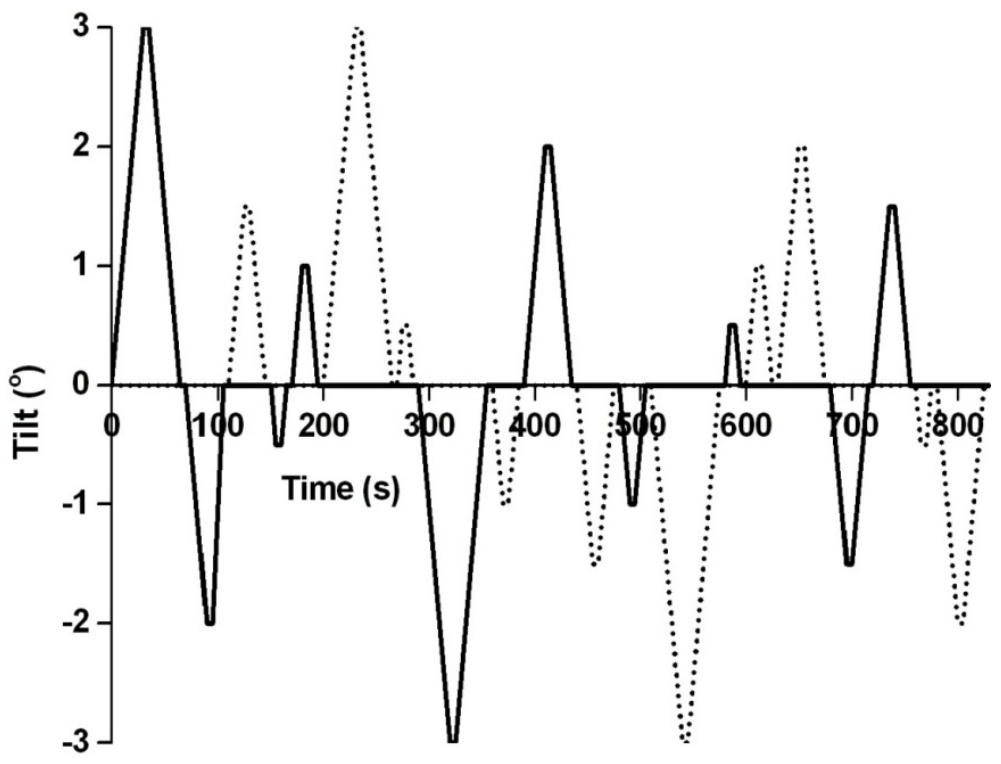

Figure C.8: Tilt platform profile with $0.1 \%$ tilts of $0.5,1.0,1.5,2.0$ and $3.0^{\circ}$ from and towards absolute horizontal platform orientation. Voice commands were given just before an orientation change. Positive angles are right- or forward. Solid: right- and leftward, dashed: for- and backward.

Concerning the profile, it might have been theoretically possible for the subjects to derive direction perception through rational pondering (e.g. a leftward tilt from absolute horizontal was always followed by a rightward tilt). However, subjects in our experiments were not told this would happen and there was no evidence that this might be the case. Small tilts were not perceived very well, making this pondering very difficult. Moreover, the first stimulus was a rightward tilt which was followed by 2 leftward tilts. Finally, subjective reports from our subjects indicated that tilt perception during these slow movements was based on bodily sensations rather than on rational estimates. 


\section{Data analysis}

After having tested the subjects on centrifugation, on the linear sled and on the tilt platform, they were asked in an open question to indicate their cues for stimulus directions.

The response rates, defined as the percentage of correctly detected stimuli, or the angles at which tilt direction was perceived, were compared between stimuli using the Friedman non-parametric repeated measures test, and if it reached significance it was followed by a post-hoc Wilcoxon signed rank test with Tukey correction for multiple testing to determine possible:

- Unilateral centrifugation; differences between tilt directions and eccenter velocities

- Bilateral centrifugation; the difference between acceleration and deceleration stimuli

- Linear sled; differences between stimulus velocities and AP and LR stimuli

- Tilt platform; differences between 1. Left- and rightward stimuli, 2. For- and backward stimuli and 3. Stimuli from and towards absolute horizontal platform orientation.

The level of significance in all tests was set at $p<0.05$. Average thresholds and $95 \%$ confidence intervals using percentiles were determined. Intra and inter-individual variability was defined as the standard deviation relative to the average threshold, the former averaged over the 3 subjects selected for 5 runs.

\subsection{Results}

\section{Centrifugation}

\section{Unilateral}

The angles at which tilt direction was perceived were not significantly different $(p>0.4)$ for left- or rightward tilts or the 3 eccenter velocities during unilateral centrifugation and therefore averaged per individual. The average threshold $\left(3.6^{\circ}\right)$ and confidence interval $\left(1.9-5.6^{\circ}\right)$ at which tilt direction was perceived are shown in figure C.9. Inter-individual variability was $25 \%$, intra-individual variability was on average $11 \%$. 


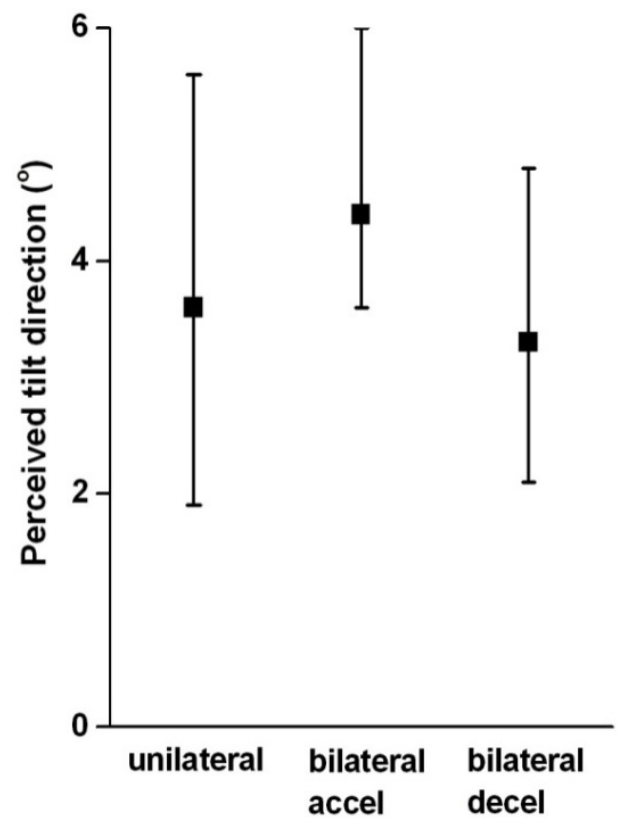

Figure C.9: Mean and 95\% confidence intervals for the angles at which tilt direction was perceived during unilateral and bilateral centrifugation. The differences between unilateral and bilateral acceleration as well as bilateral acceleration (accel) and deceleration (decel) were both significant $(p<0.01)$, the difference between unilateral and bilateral deceleration was not significant $(p>$ 0.6).

\section{Bilateral}

The difference between acceleration and deceleration angles at which tilt direction was perceived during bilateral centrifugation was significant $(p<0.01)$. The average thresholds $\left(4.4^{\circ}\right.$ and $3.3^{\circ}$ respectively) and confidence intervals $\left(3.6-6.0^{\circ}\right.$ and $2.1-$ $\left.4.8^{\circ}\right)$ are shown in figure C.9. Inter-individual variability was $13 \%$ and $21 \%$ respectively, intra-individual variability was on average $7 \%$.

The angles at which tilt direction was perceived was significantly different $(p<0.01)$ for unilateral (confidence interval $1.9-5.6^{\circ}$ ) versus bilateral acceleration (confidence interval $\left.3.6-6.0^{\circ}\right)$ and not significantly different $(p>0.6)$ for unilateral versus bilateral deceleration (confidence interval $2.1-4.8^{\circ}$ ).

All subjects indicated that they had no cues for tilt direction detection during centrifugation other than a whole body tilt sensation. 


\section{Linear sled}

The low accelerations $\left(5 \mathrm{~cm} / \mathrm{s}^{2}\right)$ had significantly $(p<0.01)$ lower response rates than the high accelerations $\left(10\right.$ and $20 \mathrm{~cm} / \mathrm{s}^{2}$ ). The 4 stimuli with $5 \mathrm{~cm} / \mathrm{s}^{2}$ (low) accelerations and the 5 stimuli with 10 and $20 \mathrm{~cm} / \mathrm{s}^{2}$ (high) accelerations were however mutually not significantly different $(p>0.4)$, nor were the AP and LR stimuli $(p>0.6)$. Therefore the response rates were averaged for each subject and calculated separately for low (5 $\mathrm{cm} / \mathrm{s}^{2}$ ) and high (10 and $20 \mathrm{~cm} / \mathrm{s}^{2}$ ) accelerations. The average response rates (68\% (low) and $86 \%$ (high)) and confidence intervals (33-96\% (low) and $56-98 \%$ (high)) are shown in figure C.10.

Inter-individual variability was $23 \%$ and $12 \%$ respectively, intra-individual variability was on average $3 \%$. Average response rates (confidence intervals) for naso-occipital (AP) and interaural stimuli (LR) only are $68 \%(29-100 \%)$ for low AP, $83 \%(63-100 \%)$ for high AP, $68 \%$ (38-100\%) for low LR and $87 \%$ (46-100\%) for high LR.

13 subjects indicated that their cues for stimulus direction (sled deceleration) were pressure changes on the body surface (in other words movement perception), only 2 subjects indicated the perception of whole body tilt as a cue.

\section{Tilt platform}

Response rates for the 5 tilt angles in 4 directions (left, right, for- and backward) from and towards horizontal were significantly different $(p=0.000)$. Tilts from and towards horizontal were not significantly different $(p>0.2)$ and therefore these responses were averaged. Response rates for LR and AP tilts were significantly different for $0.5^{\circ}(p<$ $0.02)$ and $1.5^{\circ}(p=0.05)$ tilts, but not for 1,2 and $3^{\circ}$ tilts, and therefore $L R$ and AP responses were not averaged.

- For LR tilts response rates were not significantly different for $0.5,1$ and $2^{\circ}$ tilts or for 1.5 and $3^{\circ}$ tilts, but the former were significantly $(p<0.05)$ lower than the latter

- For AP tilts response rates were not significantly different for $1,1.5,2$ and $3^{\circ}$ tilts, the response rate for $0.5^{\circ}$ tilts was significantly $(p<0.02)$ lower than the other response rates

The average response rates and confidence intervals for both LR and AP tilts are shown in figure C.11. 
All subjects indicated that their main cues for perception of platform tilt direction were reflexive body reactions to compensate body tilts and pressure changes under their buttocks on the chair.

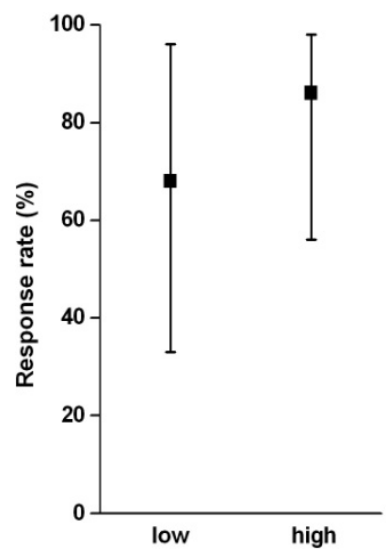

Figure C.10: $95 \%$ confidence intervals for the correct response rates for low $\left(5 \mathrm{~cm} / \mathrm{s}^{2}\right)$ and high (10 and $20 \mathrm{~cm} / \mathrm{s}^{2}$ ) accelerations on the linear sled. The difference between low and high accelerations was significant $(p<0.01)$

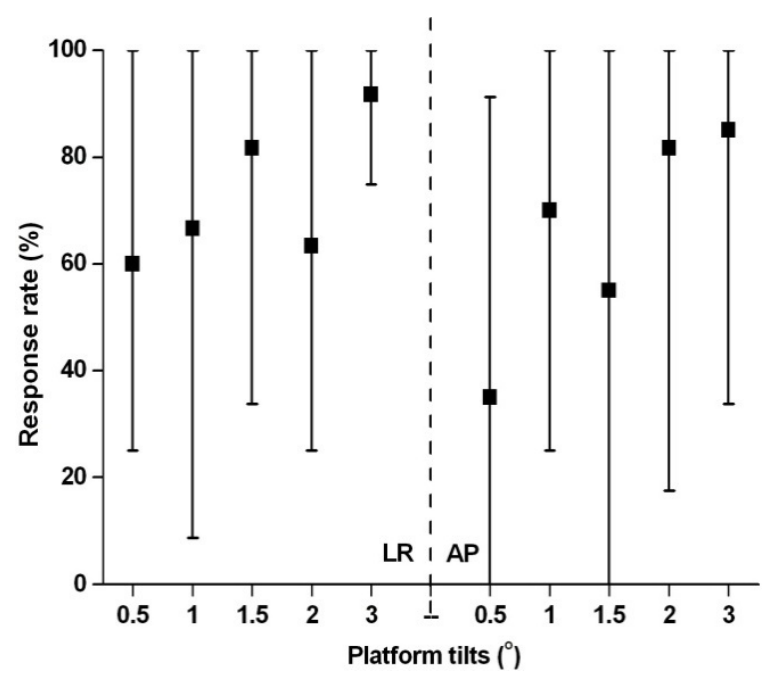

Figure C.11: 95\% confidence intervals for the correct response rates for $L R$ and AP tilts on the tilt platform $\left(0.1^{\circ} / \mathrm{s}\right)$ for $0.5,1.0,1.5,2.0$ and $3.0^{\circ}$ tilts. For $L R$ tilts response rates for $0.5,1.0$ and 2.0 ${ }^{\circ}$ tilts were significantly $(p<0.05)$ lower than for 1.5 and $3.0^{\circ}$ tilts. For AP tilts response rates for $0.5^{\circ}$ tilts were significantly $(p<0.02)$ lower than for $1.0,1.5,2.0$ and $3.0^{\circ}$ tilts. 


\subsection{Discussion}

In this study, thresholds for perception of tilt and translation were investigated using 3 new motion/tilt profile paradigms; tilt perception during centrifugation, tilt perception during support-phase tilts and translation perception during whole body accelerations.

\section{Centrifugation}

During unilateral and bilateral eccentric rotations subjects were instructed to verbally indicate the perceived tilt direction, which mainly depends on otolithic function, because all subjects had no cues for tilt direction detection other than a whole body tilt sensation. Our results indicate a threshold for perception of tilt during centrifugation of about $2^{\circ}$ with a substantial inter-individual range $\left(1.9-5.6^{\circ}\right)$ for healthy subjects. Bringoux et al. [Bringoux et al., 2002] found a similar threshold - about $1.5^{\circ}$ - in both healthy subjects and bilateral vestibular areflexia patients while seated and immobilized during $0.05 \%$ tilts. Their results might have been negatively influenced by tilt adaptation, although their results were not significantly different from ours. The results from Bisdorff et al. [Bisdorff et al., 1996], who investigated tilt direction perception in healthy subjects while seated in a padded chair during $1.5 \%$ tilts, were significantly ( $p$ $<0.001$ ) higher than ours, probably because of the time to respond in relation to the applied tilt velocity. Teasdale et al. [Teasdale et al., 1999] found a significantly $(p<0.01)$ lower threshold of about $0.5^{\circ}$ while standing against an L-shaped vertical platform without body fixation during $0.05 \%$ tilts, a situation which is however dominated by somatosensory input.

\section{Linear sled}

Whole body left-right and fore-aft decelerations opposite to the actual linear velocity direction while seated were applied during continuous subthreshold linear accelerations to induce vestibular translation/tilt perceptions. The response rate confidence intervals for low $\left(5 \mathrm{~cm} / \mathrm{s}^{2}\right.$ ) accelerations (average $68 \%, \mathrm{Cl} 33-96 \%$ ) were significantly lower than for high (10 and $20 \mathrm{~cm} / \mathrm{s}^{2}$ ) accelerations (average $86 \%, \mathrm{Cl} 56-98 \%$ ), and we found no significant directional preponderance just as Benson et al. [Benson et al., 1986]. We could not find consistent results within stimuli expressed in terms of velocity [Kingma, 2005]. 
Melvill Jones and Young [Melvill Jones and Young, 1978] found the threshold to be about $15 \mathrm{~cm} / \mathrm{s}$, based on response latencies to acceleration steps. In practice however all subjects responded in their study on all possible occasions at acceleration magnitudes above $15 \mathrm{~cm} / \mathrm{s}^{2}$ ( $0.9^{\circ}$ of tilt), which seems to be in line with our results. Nevertheless, the responses mainly depended on somatosensory information, because most of our subjects indicated pressure changes as indication for stimulus direction.

\section{Tilt platform}

During discrete slow velocity platform roll and pitch tilts subjects were instructed while seated to verbally indicate the perceived tilt direction. The response rate confidence intervals had a substantial inter-individual range for healthy subjects with the lower limit on average $24 \%$. The low response rate for most platform tilts might be caused by an unconscious body orientation compensation in the direction opposite to the platform rotation, as was previously shown by Teasdale et al. [Teasdale et al., 1999]. Nevertheless, just as for the linear sled, the responses mainly depended on somatosensory signals, because all our subjects indicated that their main cues for perception of platform tilt direction were antigravity muscular activity and pressure changes under their buttocks.

\subsection{Conclusion}

According to the reports of our healthy subjects, tilt perception during eccentric rotations seems to depend on vestibular, i.e. otolithic function. Following the subjects' sensations, movement perception during whole body decelerations on a linear sled and tilt perception upon discrete platform tilts mainly depends on somatosensory information, suggesting a dominant role of the somatosensory and proprioceptive system.

So perception of platform tilts and perception of movement on a linear sled might be more appropriate to detect thresholds for the somatosensory / proprioceptive systems, but less appropriate to detect thresholds for the vestibular system. Tilt sensations during eccentric rotations seems to be the most promising perception tool for the evaluation of utricular dysfunction [Clement and Deguine, 2010], with a threshold in healthy subjects ranging between 1.9 and $5.6^{\circ}$. 


\section{References}

Anastasopoulos, D., Bronstein, A., Haslwanter, T., Fetter, M. \& Dichgans, J. (1999) The role of somatosensory input for the perception of verticality. Ann N Y Acad Sci, 871, 379-83.

Anastasopoulos, D., Haslwanter, T., Bronstein, A., Fetter, M. \& Dichgans, J. (1997) Dissociation between the perception of body verticality and the visual vertical in acute peripheral vestibular disorder in humans. Neurosci Lett, 233, 151-3.

Aoki, M., Ito, Y., Burchill, P., Brookes, G. B. \& Gresty, M. A. (1999) Tilted perception of the subjective 'upright' in unilateral loss of vestibular function. Am J Otol, 20, 741-7.

Benson, A. J., Diaz, E. \& Farrugia, P. (1975) The perception of body orientation relative to a rotating linear acceleration vector. Fortschr Zool, 23, 264-74.

Benson, A. J., Hutt, E. C. \& Brown, S. F. (1989) Thresholds for the perception of whole body angular movement about a vertical axis. Aviat Space Environ Med, 60, 205-13.

Benson, A. J., Spencer, M. B. \& Stott, J. R. (1986) Thresholds for the detection of the direction of whole-body, linear movement in the horizontal plane. Aviat Space Environ Med, 57, 1088-96.

Bisdorff, A. R., Wolsley, C. J., Anastasopoulos, D., Bronstein, A. M. \& Gresty, M. A. (1996) The perception of body verticality (subjective postural vertical) in peripheral and central vestibular disorders. Brain, 119 ( Pt 5), 1523-34.

Bringoux, L., Nougier, V., Barraud, P. A., Marin, L. \& Raphel, C. (2003) Contribution of somesthetic information to the perception of body orientation in the pitch dimension. $Q J$ Exp Psychol A, 56, 909-23.

Bringoux, L., Schmerber, S., Nougier, V., Dumas, G., Barraud, P. A. \& Raphel, C. (2002) Perception of slow pitch and roll body tilts in bilateral labyrinthine-defective subjects. Neuropsychologia, 40, 367-72.

Bronstein, A. M. (1999) The interaction of otolith and proprioceptive information in the perception of verticality. The effects of labyrinthine and CNS disease. Ann N Y Acad Sci, 871, 324-33.

Ceyte, H., Cian, C., Nougier, V., Olivier, I. \& Trousselard, M. (2007) Role of gravity-based information on the orientation and localization of the perceived body midline. Exp Brain Res, 176, 504-9.

Clark, B. (1967) Thresholds for the perception of angular acceleration in man. Aerosp Med, 38, 443-50.

Clark, B. \& Graybiel, A. (1963) Perception of the postural vertical in normals and subjects with labyrinthine defects. J Exp Psychol, 65, 490-4.

Clark, B. \& Graybiel, A. (1964) Perception of the Postural Vertical Following Prolonged Bodily Tilt in Normals and Subjects with Labyrinthine Defects. Acta Otolaryngol, 58, 143-8.

Clark, B. \& Stewart, J. D. (1970) Thresholds for the perception of angular acceleration about the three major body axes. Acta Otolaryngol, 69, 231-8.

Clark, B., Stewart, J. D. \& Phillips, N. H. (1980) Thresholds for detection of constant rotary acceleration during vibratory rotary acceleration. Aviat Space Environ Med, 51, 603-6.

Clarke, A. H., Schonfeld, U. \& Helling, K. (2003) Unilateral examination of utricle and saccule function. J Vestib Res, 13, 215-25. 
Clement, G. \& Deguine, O. (2010) Perception of tilt and ocular torsion of vestibular patients during eccentric rotation. Neurosci Lett, 468, 161-5.

Fernandez, C. \& Goldberg, J. M. (1976) Physiology of peripheral neurons innervating otolith organs of the squirrel monkey. I. Response to static tilts and to long-duration centrifugal force. J Neurophysiol, 39, 970-84.

Fitzpatrick, R. \& Mccloskey, D. I. (1994) Proprioceptive, visual and vestibular thresholds for the perception of sway during standing in humans. J Physiol, 478 ( Pt 1), 173-86.

Gianna, C., Heimbrand, S. \& Gresty, M. (1996) Thresholds for detection of motion direction during passive lateral whole-body acceleration in normal subjects and patients with bilateral loss of labyrinthine function. Brain Res Bull, 40, 443-7; discussion 448-9.

Grabherr, L., Nicoucar, K., Mast, F. W. \& Merfeld, D. M. (2008) Vestibular thresholds for yaw rotation about an earth-vertical axis as a function of frequency. Exp Brain Res, 186, 67781.

Groen, E. L. \& Bles, W. (2004) How to use body tilt for the simulation of linear self motion. J Vestib Res, 14, 375-85.

Grunfeld, E. A., Okada, T., Jauregui-Renaud, K. \& Bronstein, A. M. (2000) The effect of habituation and plane of rotation on vestibular perceptual responses. $J$ Vestib Res, 10, 193-200.

Guedry, F. E. (1974) Vestibular System part 2: Psychophysics, Applied Aspects and General Interpretations. IN KORNHUBER, H. H. (Ed.). Berlin - Heidelberg - New York, SpringerVerlag.

Guenther, A. L., Bartl, K., Nauderer, J., Schneider, E., Huesmann, A., Brandt, T. \& Glasauer, S. (2009) Modality-dependent indication of the subjective vertical during combined linear and rotational movements. Ann N Y Acad Sci, 1164, 376-9.

Halmagyi, G. M. \& Curthoys, I. S. (1988) A clinical sign of canal paresis. Arch Neurol, 45, 737-9.

Holly, J. E., Wood, S. J. \& Mccollum, G. (2009) Phase-linking and the perceived motion during offvertical axis rotation. Biol Cybern.

Ito, Y. \& Gresty, M. A. (1996) Shift of subjective reference and visual orientation during slow pitch tilt for the seated human subject. Brain Res Bull, 40, 417-21.

Jarchow, T. \& Mast, F. W. (1999) The effect of water immersion on postural and visual orientation. Aviat Space Environ Med, 70, 879-86.

Kanayama, R., Bronstein, A. M., Gresty, M. A., Brookes, G. B., Faldon, M. E. \& Nakamura, T. (1995) Perceptual studies in patients with vestibular neurectomy. Acta Otolaryngol Suppl, 520 Pt 2, 408-11.

Kingma, H., Kavelaars, J., Van Tienen, N. \& Caris, R. (2001) Evaluation of the statolith function by measurement of ocular counterrolling? Oto-Rhino-Laryngologia Nova, 11, 68-79.

Kingma, H. (2005) Thresholds for perception of direction of linear acceleration as a possible evaluation of the otolith function. BMC Ear Nose Throat Disord, 5, 5.

Kingma, H. (2006) Function tests of the otolith or statolith system. Curr Opin Neurol, 19, 21-5.

Lopez, C., Lacour, M., Ahmadi, A. E., Magnan, J. \& Borel, L. (2007) Changes of visual vertical perception: a long-term sign of unilateral and bilateral vestibular loss. Neuropsychologia, $45,2025-37$. 
Mahboobin, A., Loughlin, P., Atkeson, C. \& Redfern, M. (2009) A mechanism for sensory reweighting in postural control. Med Biol Eng Comput.

Mazibrada, G., Tariq, S., Perennou, D., Gresty, M., Greenwood, R. \& Bronstein, A. M. (2008) The peripheral nervous system and the perception of verticality. Gait Posture, 27, 202-8.

Melvill-Jones, G. (1979) Biophysics of the Peripheral End Organs. IN WILSON, V. J. \& MELVILLJONES, G. (Eds.) Mammalian vestibular physiology. New York, Plenum Press.

Melvill Jones, G. (1979) Biophysics of the Peripheral End Organs. IN WILSON, V. J. \& MELVILLJONES, G. (Eds.) Mammalian vestibular physiology. New York, Plenum Press.

Melvill Jones, G. \& Young, L. R. (1978) Subjective detection of vertical acceleration: a velocitydependent response? Acta Otolaryngol, 85, 45-53.

Merfeld, D. M., Park, S., Gianna-Poulin, C., Black, F. O. \& Wood, S. (2005a) Vestibular perception and action employ qualitatively different mechanisms. I. Frequency response of VOR and perceptual responses during Translation and Tilt. J Neurophysiol, 94, 186-98.

Merfeld, D. M., Park, S., Gianna-Poulin, C., Black, F. O. \& Wood, S. (2005b) Vestibular perception and action employ qualitatively different mechanisms. II. VOR and perceptual responses during combined Tilt\&Translation. J Neurophysiol, 94, 199-205.

Metcalfe, T. \& Gresty, M. (1992) Self-controlled reorienting movements in response to rotational displacements in normal subjects and patients with labyrinthine disease. Ann NY Acad Sci, 656, 695-8.

Mittelstaedt, H. (1999) The role of the otoliths in perception of the vertical and in path integration. Ann N Y Acad Sci, 871, 334-44.

Mittelstaedt, M. L. \& Mittelstaedt, H. (1997) The effect of centrifugal force on the perception of rotation about a vertical axis. Naturwissenschaften, 84, 366-9.

Nelson, J. G. (1968) Effect of water immersion and body position upon perception of the gravitational vertical. Aerosp Med, 39, 806-11.

Nooij, S. A. (2008) Vestibular adaptation to an altered gravitational environment: Consequences for spatial orientation, Delft, University of Technology. ISBN 978-90-9022982-9.

Nowe, V., Wuyts, F. L., Hoppenbrouwers, M., Van De Heyning, P. H., De Schepper, A. M. \& Parizel, P. M. (2003) The interutricular distance determined from external landmarks. J Vestib Res, 13, 17-23.

Okada, T., Grunfeld, E., Shallo-Hoffmann, J. \& Bronstein, A. M. (1999) Vestibular perception of angular velocity in normal subjects and in patients with congenital nystagmus. Brain, 122 ( Pt 7), 1293-303.

Raphel, C. \& Barraud, P. A. (1994) Perceptual thresholds of radial accelerations as indicated by visually perceived eye level. Aviat Space Environ Med, 65, 204-8.

Riccio, G. E., Martin, E. J. \& Stoffregen, T. A. (1992) The role of balance dynamics in the active perception of orientation. J Exp Psychol Hum Percept Perform, 18, 624-44.

Rodenburg, M., Stassen, H. P. \& Maas, A. J. (1981) The threshold of perception of angular acceleration as a function of duration. Biol Cybern, 39, 223-6.

Roggeveen, L. J. \& Nijhoff, P. (1956) The normal and pathological threshold of the perception of angular accelerations for the optogyral illusion and the turning sensation. Acta Otolaryngol, 46, 533-41. 
Ross, H. E., Crickmar, S. D., Sills, N. V. \& Owen, E. P. (1969) Orientation to the vertical in free divers. Aerosp Med, 40, 728-32.

Schmid-Priscoveanu, A., Bohmer, A., Obzina, H. \& Straumann, D. (2001) Caloric and search-coil head-impulse testing in patients after vestibular neuritis. J Assoc Res Otolaryngol, 2, 728.

Seemungal, B.M., Gunaratne, I.A., Fleming, I.O., Gresty, M.A., Bronstein, A.M. (2004) Perceptual and nystagmic thresholds of vestibular function in yaw. J Vest Res 14 (6), 2004, 461-66.

Seidman, S. H., Au Yong, N. \& Paige, G. D. (2009) The perception of translational motion: what is vestibular and what is not. Ann N Y Acad Sci, 1164, 222-8.

Teasdale, N., Nougier, V., Barraud, P. A., Bourdin, C., Debu, B., Poquin, D. \& Raphel, C. (1999) Contribution of ankle, knee, and hip joints to the perception threshold for support surface rotation. Percept Psychophys, 61, 615-24.

Tribukait, A. \& Eiken, O. (2006) Changes in the perceived head transversal plane and the subjective visual horizontal induced by Coriolis stimulation during gondola centrifugation. J Vestib Res, 16, 105-16.

Trousselard, M., Cian, C., Nougier, V., Pla, S. \& Raphel, C. (2003) Contribution of somesthetic cues to the perception of body orientation and subjective visual vertical. Percept Psychophys, $65,1179-87$.

Vaitl, D., Mittelstaedt, H., Saborowski, R., Stark, R. \& Baisch, F. (2002) Shifts in blood volume alter the perception of posture: further evidence for somatic graviception. Int J Psychophysiol, 44, 1-11.

Vaugoyeau, M., Viel, S., Amblard, B., Azulay, J. P. \& Assaiante, C. (2008) Proprioceptive contribution of postural control as assessed from very slow oscillations of the support in healthy humans. Gait Posture, 27, 294-302.

Vingerhoets, R. A., De Vrijer, M., Van Gisbergen, J. A. \& Medendorp, W. P. (2009) Fusion of visual and vestibular tilt cues in the perception of visual vertical. J Neurophysiol, 101, 1321-33.

Von Brevern, M., Faldon, M. E., Brookes, G. B. \& Gresty, M. A. (1997) Evaluating 3D semicircular canal function by perception of rotation. Am J Otol, 18, 484-93.

Wright, W. G. \& Horak, F. B. (2007) Interaction of posture and conscious perception of gravitational vertical and surface horizontal. Exp Brain Res, 182, 321-32.

Yi, Y. \& Park, S. (2009) Effect of reduced cutaneous cues on motion perception and postural control. Exp Brain Res, 195, 361-9.

Zupan, L. H. \& Merfeld, D. M. (2008) Interaural self-motion linear velocity thresholds are shifted by roll vection. Exp Brain Res, 191, 505-11. 


\section{Part D}

EYE MOVEMENT QUANTIFICATION
Over the last decades, small head-mounted video eye trackers have been developed in order to record

eye movements. Real-time systems - with a low sampling frequency of 50/60 Hz-are used for

clinical vestibular practice, but are generally considered not to be suited for measuring fast eye

movements.

In this part it will be shown that saccadic eye movements, having an amplitude of at least $5^{\circ}$, can

in good approximation be considered to be bandwidth limited up to a frequency of $25-30 \mathrm{~Hz}$. Using the Nyquist theorem to reconstruct saccadic

eye movement signals at higher temporal resolutions, it was shown that accurate values for saccade peak velocities, recorded at $50 \mathrm{~Hz}$, can be

obtained, but saccade peak accelerations and decelerations cannot. Additionally, for detection of eye saccadic amplitudes, video oculography (VOG) and the scleral search coil (SSC) technique are the preferred techniques, because substantial drift makes electro nystagmography ENG less appropriate. VOG and ENG are the preferred techniques to measure eye peak velocities, because of lens slippage of the search coil and slowing down of the eye dynamics by the presence of the search coil in SSC.

In conclusion: video-eyetrackers sampling at 50/60 $\mathrm{Hz}$ are appropriate for detecting the clinical relevant saccade peak velocities in contrast to what has been stated up till now, when the data is interpolated to 1 $\mathrm{kHz}$. Online real-time VOG with a sampling frequency

of minimal $50 \mathrm{~Hz}$ using the Nyquist sampling theorem to interpolate the data to $1 \mathrm{kHz}$ appears to be the preferred clinical technique for measurement of saccadic amplitudes and velocities. 
This part would not have been possible without the work from my graduate students Anne Vrijling, Roel Wierts, Mariken Zijlmans and Mark Pijnenburg, the help from Stephan Jansen and the technical support from Iwan de Jong, René Hermans and Erik Brands.

Based on

Wierts $R$, Janssen MJA and Kingma $H$. Measuring saccade peak velocity using a lowfrequency sampling rate of $50 \mathrm{~Hz}$. IEEE Trans Biomed Eng 2008; 55: 2840-2 
Measurement of eye movements plays an important role in testing the oculomotor and vestibular system. In order to quantify and observe eye movements, several eye movement recording techniques have been developed. The techniques most frequently used in clinical practice at current are electro nystagmography (ENG), infrared light reflexes oculography and video oculography (VOG). The semi-invasive scleral search coil technique is also used (SSC) and is considered as the gold standard of eye movement recording, but is mainly applied in research or in patients with a complex abnormalities or when calibration necessary for ENG and VOG is not possible [Kessler et al., 2008].

For many years ENG has been the most applied technique in clinical practice for detection of horizontal and vertical eye movements for the following reasons:

1. ENG is non-invasive and is generally well tolerated by patients of all ages

2. the signal can theoretically be sampled at high frequencies $(300-1000 \mathrm{~Hz})$ as long as the signal-noise ratio does not force the use of high-frequency cut-off filtering

3. ENG allows detection of eye movements over large angles of gaze $\left( \pm 80^{\circ}\right.$ for horizontal and $\pm 50^{\circ}$ for vertical eye movements [Young and Sheena, 1975, Kumar and Krol, 1992]) although linearity is only preserved over angles smaller than 25 degrees

4. ENG recordings can even be obtained when eyes are closed.

Disadvantages of ENG are that

1. eye torsion cannot be measured

2. the magnitude of the ENG signal depends on the cornea-retinal potential which changes with the illumination of the retina which makes it necessary to calibrate frequently and to avoid large changes in the ambient illumination of the room [North, 1965].

3. due to the anatomy the electrodes have to be fixed always at an asymmetric position relative to the eye which results in non-linearities in monocular recordings.

4. the presence of noise, caused by muscle activity, ECG, eyelid movement and changes in skin impedance (e.g. transpiration) requires the signal to be filtered, which results in a limited bandwidth of about $60 \mathrm{~Hz}$ [Reulen et al., 1988]. 
The SSC technique was introduced by Robinson [Robinson, 1963] and modified by Collewijn et al. [Collewijn et al., 1975]. It is considered to be the gold standard for the measurement of eye movements in three dimensions because of its high spatial accuracy ( $<1^{\prime}$ of arc) and temporal resolution ( $<1 \mathrm{~ms}$ ) [Collewijn et al., 1975, van der Geest and Frens, 2002]. However, a major drawback of this technique is the need for the patient to wear a relatively large contact lens that is not customized for the individual, and is held to the eye by suction, which causes irritation of the eye and limits the examination time to about 30 minutes. Therefore, the SSC technique has never gained a broad clinical application. The SSC technique has also been criticised when it was reported that slippage of the contact lens (silicone ring) over the cornea may occur especially during fast eye movements ${ }^{4}$. This has also been suggested by others [Houben et al., 2006, van der Geest and Frens, 2002, DiScenna et al., 1995] based on a tendency of higher velocities measured with the video system than for the coil system, when recording these signals simultaneously. Additionally it was shown by Frens and Van der Geest [Frens and van der Geest, 2002] that the placement of scleral search coils onto the eyes influences the kinematics of saccades, as saccades become longer and slower when a scleral search coil is mounted on the eye.

In the infrared light reflection oculography technique, infrared sensors are directed towards the middle of the nasal and temporal limbus (the transition between the eye white and the iris) of the eye that is illuminated by infrared LED's. Each sensor converts the amount of reflected light to a voltage that is a function of eye position. The method enables detection of horizontal eye movements of $30^{\circ}$ amplitude with a time resolution of $1 \mathrm{~ms}$, permitting accurate determination of latency values [Reulen et al., 1988]. However, substantial artefacts and errors can occur when the sensors and diodes are not precisely positioned relative to the limbus. Moreover, vertical eye movements can hardly be recorded as the limbus is often covered by the eye lids [Truong and Feldon, 1987]. Therefore, the method has not gained broad clinical application.

\footnotetext{
${ }^{4}$ Lens slippage of the scleral search coil was detected with a VOG system by Clarke, A. H. and shown in a poster presentation at the Bárány Society Meeting (1998).
} 
Since the development of small head-mounted video eye trackers, infra red video oculography has gained in popularity amongst clinical practitioners. The major reasons for that seem to be:

1. the easy use

2. the lack of clear artefacts as compared to ENG

3. the low noise level and high spatial accuracy

4. the fact that calibration is often not strictly necessary.

5. in many infrared VOG systems visual fixation can be completely abolished by covering the video eye tracker, so that there is no need for a complex infrastructure with a completely dark examination room any more

6. some VOG systems allow detection of torsional eye movements.

The developed head-mounted VOG systems can mainly be divided into two different groups: low-frequency sampling systems $(50-60 \mathrm{~Hz}$, [Schmid-Priscoveanu and Allum, 1999, Allum et al., 1998]) operating real-time, and high speed sampling systems up to $400 \mathrm{~Hz}$ [Clarke et al., 2002]. Major drawbacks of the latter are that 1) data is processed offline after the examination is completed - patient coaching during the examination is essential - 2) the available online systems are not useful in vestibular practice - they are unstable or uncomfortable during head movements and 3 ) relatively expensive. The drawback of real-time sampling VOG systems is the low sampling rate which is often considered to hamper a correct analysis of fast eye movements like saccades. Especially for the calculation of saccadic peak velocities, an important parameter in clinical practice, high sampling frequencies are always mentioned to be necessary. Juhola et al. stated that it is important to use a relatively high sampling frequency of at least $300 \mathrm{~Hz}$ for accurate recording of saccades [Juhola et al., 1985].

In chapter 6 saccade power spectra will be examined and a technique to improve the temporal resolution of $50 \mathrm{~Hz}$ eye movement recordings using VOG will be evaluated. In chapter 7 a quantitative and qualitative comparison of 1) the SSC technique, considered to be the gold standard for the measurement of eye movements, 2 ) the regular clinically applied ENG method, and 3) online real-time $50 \mathrm{~Hz}$ VOG using the Nyquist sampling theorem to interpolate the data to $1 \mathrm{kHz}$ [Wierts et al., 2008]. 


\section{CHAPTER 6: MEASURING SACCADE PEAK VELOCITY USING A LOW-FREQUENCY SAMPLING RATE OF $50 \mathrm{HZ}$}

The purpose of this study was to evaluate saccade power spectra and to evaluate a technique to improve the temporal resolution of $50 \mathrm{~Hz}$ eye movement recordings using VOG.

\subsection{Methods}

\section{Eye movement recordings}

Three healthy subjects without any history or evidence of ophthalmologic or neurologic disorders participated in the experiment. Ages ranged from 23 to 28 years. All subjects participated on a voluntary base after giving their informed consent.

Subjects were seated in a chair. Movement of the head was minimized using a head-rest attached to the chair. After calibration, subjects were asked to visually fixate a dot of 0.5 $\mathrm{cm}$, projected on a screen positioned $1 \mathrm{~m}$ in front of the subjects. The dot moved abruptly from side to side, forcing the subjects to make horizontal midline-crossing saccades. The angle over which the spot moved started at a small value of $5^{\circ}$ and increased in steps of $5^{\circ}$ up to an angle of $25^{\circ}$ (and $28^{\circ}$, limited by the dimensions of the screen). For each rotation angle, three saccades to the left and right were made, resulting in a total of 36 saccades per subject.

Since saccadic eye movements are the fastest eye movements one can make, they have the highest cutoff frequency of all eye movements. Thus, a sampling frequency, capable of accurately recording saccades, is also capable of recording all other types of eye movements.

Horizontal saccadic eye movements were recorded with the Skalar scleral search coil (SSC) system S3020 (Skalar Medical). The SSC signal was amplified by an analogue amplifier having a bandwidth of $200 \mathrm{~Hz}$. For the scleral search coils, the Skalar Medical combination annulus was used. The signal was recorded at a sampling frequency of 1 $\mathrm{kHz}$. 


\section{Nyquist sampling theorem}

When recording a dynamic signal, the used sampling frequency $f_{s}$ is of high importance, since a too low sampling frequency results in a loss of information, called aliasing. The Nyquist critical frequency $f_{c}$ equals half the sampling frequency $f_{s}$ [Press et al., 1995].

The Nyquist sampling theorem states that if a continuous function $x(t)$, sampled at a sampling interval $T_{s}=1 / f_{s}$, is bandwidth limited with a maximum frequency component, $\mathrm{f}_{\max }$, equal to or smaller than $f_{c}$, then the function $x(t)$ is completely determined by its samples $x[n]$ and is given explicitly by

$$
x(t)=\sum_{n=-\infty}^{+\infty} x[n] \cdot \frac{\sin \left\{2 \pi f_{c}\left(t-n T_{S}\right)\right\}}{\pi\left(t-n T_{s}\right)}
$$

with $x[n]$ given by

$$
x[n]=x\left(n T_{S}\right), n=0, \pm 1, \pm 2, \pm 3, \ldots
$$

Also, the Nyquist sampling theorem states that in order to prevent aliasing, the (continuous) signal has to be bandwidth limited with the upper frequency equal to or smaller than $f_{c}$.

The power spectra presented in this paper were obtained using a method similar to Harris et al. [Harris et al., 1990].

\subsection{Results}

\section{Spectra of saccades}

In figure D.1 the power spectra are shown for saccades of varying amplitude, showing that at low frequencies there is a constant roll-off of $20 \mathrm{~dB} /$ decade, reflecting the overall step change in eye position [Harris et al., 1990]. The power spectra of two idealized instantaneous saccades with amplitudes of $5^{\circ}$ and $28^{\circ}$ are represented by the dotted lines in figure D.1.

The fact that saccades do not occur instantaneously results in a downward departure from the theoretical $20 \mathrm{~dB} /$ decade roll-off at a frequency of $20-30 \mathrm{~Hz}$, indicating a lack of energy of higher frequency components. Since larger amplitude saccades have longer 
durations, this departure begins at lower frequencies as the amplitude of the saccade increases [Harris et al., 1990]. This indicates that for the small amplitude saccades the higher frequency components are relatively more important than for the large amplitude saccades. The lack of energy at high frequencies, together with the 20 $\mathrm{dB} /$ decade roll-off, indicates that for the accurate description of saccades the high frequency components are negligible compared to the low frequency components. This means that saccadic eye movements are in good approximation bandwidth limited with an upper frequency of $25-30 \mathrm{~Hz}$.

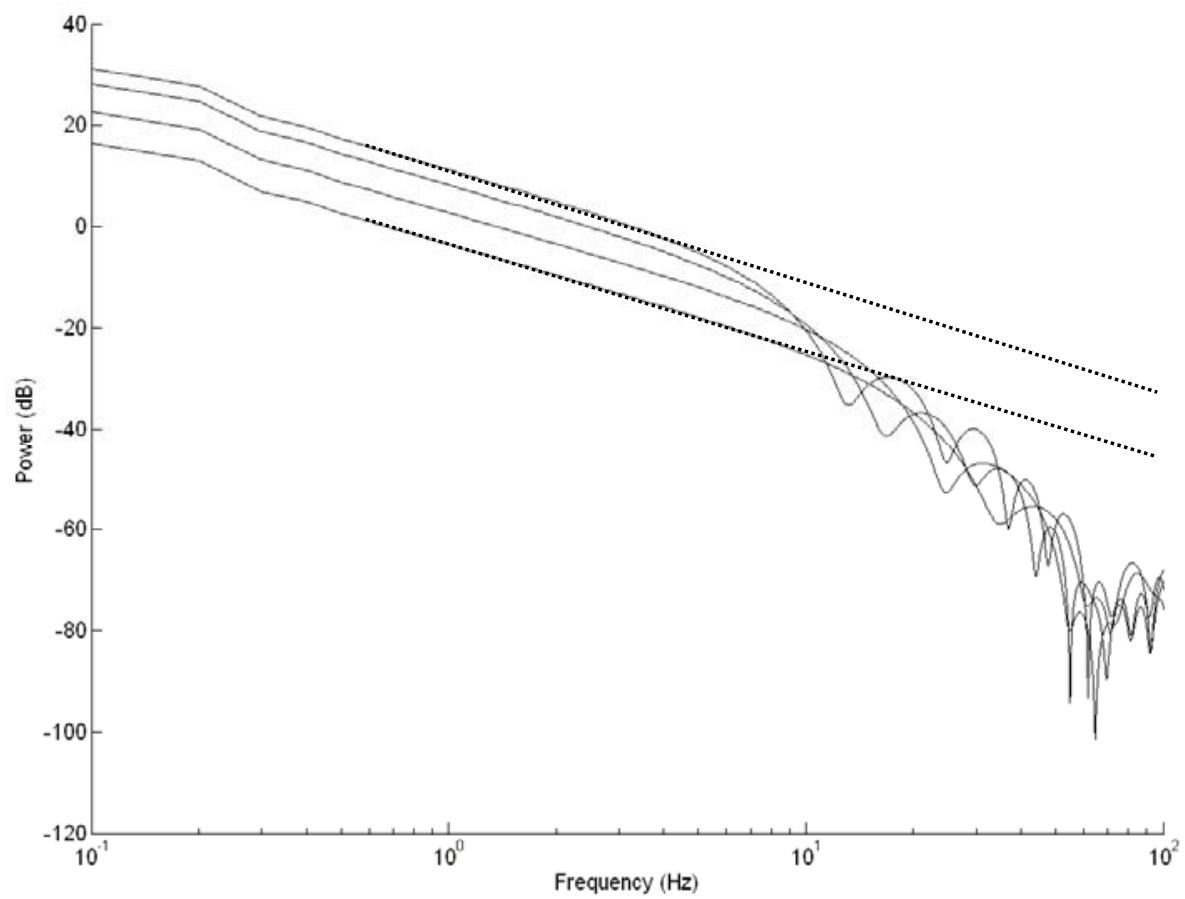

Figure D.1: Power spectra of saccades of varying amplitude. Top to bottom: $28^{\circ}, 20^{\circ}, 10^{\circ}$ and $5^{\circ}$. The dotted lines represent the power spectra of the idealized, instantaneous saccades with corresponding amplitudes. 


\section{Reconstruction technique}

As a result of this bandwidth limit, the Nyquist theorem states that a sampling frequency of $50-60 \mathrm{~Hz}$ is sufficiently high to avoid aliasing. Moreover, the eye position for times inbetween two consecutive sampling points can be obtained using equation (1). As a result, the original signal can be reconstructed at higher "sampling" frequencies. In order to show this, the saccadic eye position signal recorded at a frequency of $1 \mathrm{kHz}$ was decimated to a frequency of $50 \mathrm{~Hz}$. The obtained signal can be considered to be obtained by direct sampling of the eye position at a frequency of $50 \mathrm{~Hz}$. Thereafter, the original eye position signal was reconstructed (interpolated to the original sampling frequency of $1 \mathrm{kHz}$ ) using equation (1). From each eye position signal, also the corresponding eye velocity signal was calculated using the two point central difference differentiation algorithm. Usui and Amidror [Usui and Amidror, 1982] showed this method to be not only very practical for use, but even almost optimum. In order to avoid the differentiation of high frequency noise, a spread of six was used [Bahill et al., 1982].

In figure D.2 the measured peak velocities of both the original and reconstructed signals for one subject are shown as a function of the saccade amplitude, the so-called main sequence [Bahill et al., 1975].From this figure it can be seen that for saccades, having amplitudes in the range of $5^{\circ}$ to $28^{\circ}$, there is a good agreement between the saccade peak velocity determined for the original and reconstructed signals. For the small amplitude saccades of about $5^{\circ}$, an underestimation of the peak velocity of 10-15 $\%$ was found, shown in the inset of figure D. 2 for all subjects. For the larger amplitude saccades, the deviation of peak velocity was $3 \%$ on average, without a preference for under- or overestimation. Typical VOG noise of $0.05^{\circ}$ results in an additional error of 0.5 $\%$, independent of the saccade amplitude. Furthermore, for correction saccades present in the signal, having an amplitude smaller than $5^{\circ}$, it was observed that the reconstruction technique often did not result in reliable position and velocity signals resulting in a large underestimation of the peak velocity. And also, ringing occurs at the start and end of the saccade, which shows that the eye acceleration and decelerations cannot be detected or reconstructed accurately with sampling rates of $50-60 \mathrm{~Hz}$ or less. 


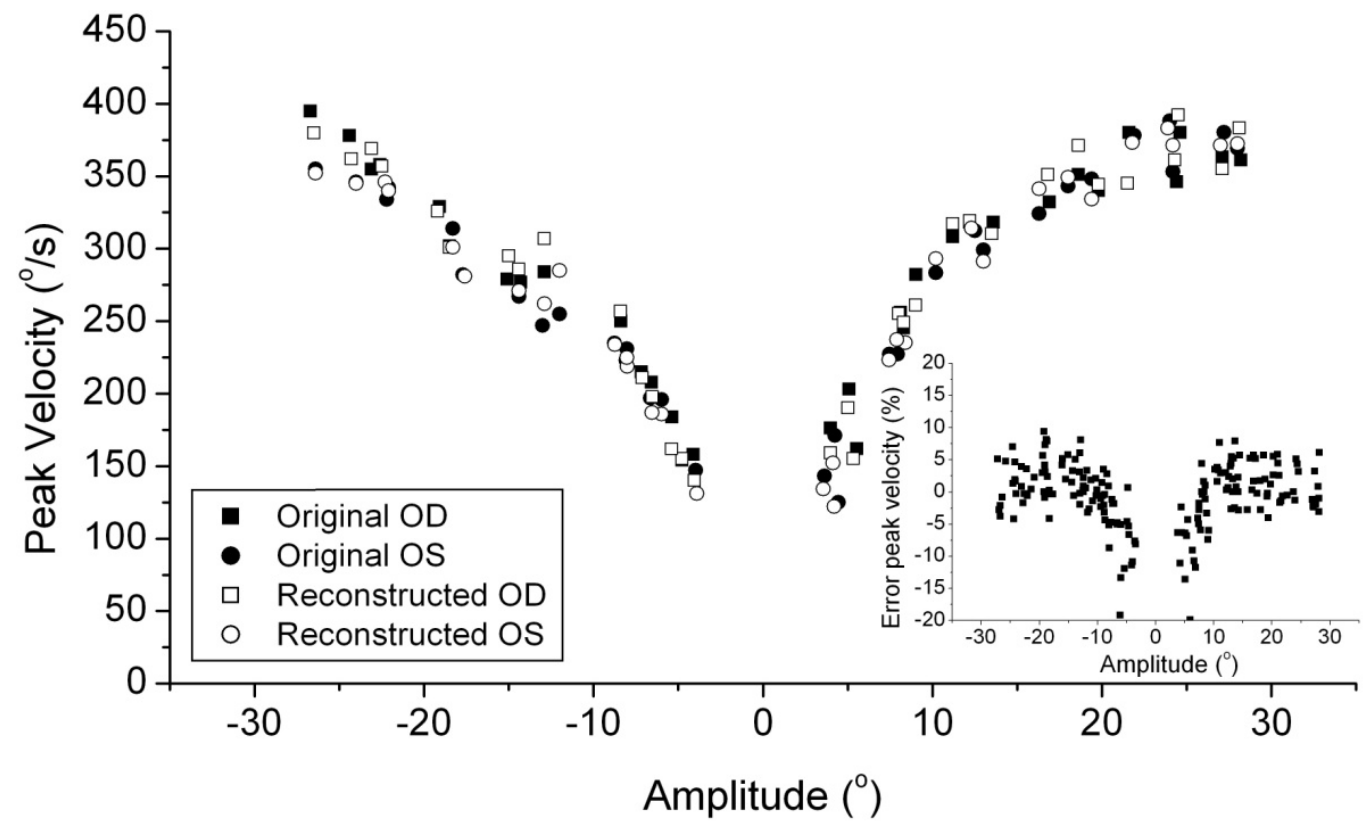

Figure D.2: Main sequence of horizontal midline-crossing saccades of the left (OS) and right (OD) eye from one subject. The filled symbols correspond to the original signal, the empty symbols with the reconstructed signal. Positive (negative) amplitudes correspond to saccades to the right (left). The inset shows the difference in peak velocity between original and reconstructed signals vs. amplitude of all subjects.

\subsection{Discussion}

From the power spectra of saccades it was shown that saccades, having an amplitude of at least $5^{\circ}$, can be considered to be bandwidth limited with an upper frequency of 25-30 $\mathrm{Hz}$. The fact that the higher frequency components still contain some relevant information results in ringing and velocity underestimation and somewhat ill-defined saccade duration and amplitude in the reconstructed signal at small amplitude saccades. Good approximations of saccade peak velocity can be obtained, as deviations of $3 \%$ are much smaller than normal variations [Wong et al., 2006].

In this study, 3 healthy subjects were considered. Since eye velocity is not increased in case of vestibular or ophthalmologic pathology, the reconstruction technique will also yield reliable eye velocity signals in patients. 


\subsection{Conclusions}

A sampling frequency of about $50 \mathrm{~Hz}$ is sufficiently high to prevent aliasing. Furthermore, it was shown that using the Nyquist sampling theorem to interpolate the data to $1 \mathrm{kHz}$, accurate values for the peak velocity of saccades, recorded at $50 \mathrm{~Hz}$, can be obtained. From these observations it can be concluded that the presented reconstruction technique is a promising technique for improving low-frequency realtime VOG systems. 


\section{CHAPTER 7: QUANTITATIVE AND QUALITATIVE COMPARISON OF ELECTRO NYSTAGMOGRAPHY (ENG), VIDEO OCULOGRAPHY (VOG) AND SCLERAL SEARCH COIL (SSC) IN MEASURING HORIZONTAL SACCADES}

The purpose of this study was a quantitative and qualitative comparison of 1) the SSC technique, considered to be the gold standard for the measurement of eye movements, 2) the regular clinically applied ENG method, and 3) online real-time $50 \mathrm{~Hz}$ VOG using the Nyquist sampling theorem to interpolate the data to $1 \mathrm{kHz}$ [Wierts et al., 2008]. Both static (accuracy ${ }^{5}$ and sensitivity ${ }^{6}$ of absolute eye position) and dynamic (saccade amplitude and peak velocity) properties of horizontal eye movements were evaluated.

\subsection{Materials and methods}

Three healthy subjects (two male, one female) without any history or evidence of ophthalmologic or neurologic disorders participated in the experiment. Ages ranged from 23 to 28 years. All subjects participated on a voluntary basis after giving their informed consent.

Subjects were seated in a chair. Movement of the head was minimized using a head-rest attached to the chair. After calibration with $20^{\circ}$ saccades, subjects were asked to visually focus on a dot with a diameter of $5 \mathrm{~mm}$, projected on a screen positioned $1 \mathrm{~m}$ in front of the subjects. The dot moved abruptly from side to side, forcing the subjects to make horizontal midline-crossing saccades. The angle over which the spot moved started at a small value of $5^{\circ}$ and increased in steps of $5^{\circ}$ up to an angle of $25^{\circ}$ (and $28^{\circ}$, limited by the dimensions of the screen). For each angle, three saccades to the left and right were made, resulting in a total of 36 saccades per subject.

Horizontal saccadic eye movements were simultaneously recorded with SSC, ENG and VOG. SSC recordings were made using the Skalar scleral search coil (SSC) system S3020 (Skalar Medical), using the Skalar Medical combination annulus after having anesthetized the eyes. ENG signals were recorded with Balancelab 1.10 (Maastricht Instruments) using $\mathrm{Ag}-\mathrm{AgCl}$ skin electrodes, after intensive cleaning of the skin with petroleum-aether and skin scrub. The ENG leads were twined to avoid disturbing

\footnotetext{
${ }^{5}$ The closeness of a measured value to a reference value.

${ }^{6}$ The degree of reproducibility
} 
electrical currents produced by the AC magnetic fields of the SSC system. VOG signals were recorded on tape (Sony Dvcam) using a (head-mounted) clinical video eyetracker (C-VET Maastricht Instruments). All signals were analysed using Matlab version R2006b. None of the subjects had worn scleral search coils before, but all were used to wearing contact lenses. For each subject, the measurement procedure was performed twice: first while recording was simultaneously performed with the ENG and the VOG system, second while simultaneously recording using the ENG, VOG and SSC system. For 3 reasons the measurements were repeated: investigation of 1) magnetic field artifacts in ENG or VOG signals due to the presence of the magnetic fields, 2) artifacts in the VOG signal due to the SSC coil on the eye which is visible in the video images and 3) the effect of the SSC coil on the eye dynamics.

The SSC signal was amplified by an analogue amplifier having a bandwidth of $200 \mathrm{~Hz}$ and recorded at a sampling frequency of $1 \mathrm{kHz}$. The SSC velocity signal was calculated using the two-point central difference method with a spread of six to suppress noise in the velocity signal [Bahill et al., 1982].

The ENG signal was amplified by an analogue amplifier having a bandwidth of $100 \mathrm{~Hz}$, recorded at a sampling frequency of $1 \mathrm{kHz}$ and low passed filtered using a discrete Fourier transform with a cut off frequency of $25 \mathrm{~Hz}$ to filter out high frequency noise, which would deteriorate the ENG velocity signal. Because saccadic eye movements are in good approximation bandwidth limited with an upper frequency of $25-30 \mathrm{~Hz}, 25 \mathrm{~Hz}$ low pass filtering of ENG does not disturb the recorded ENG signals [Wierts et al., 2008].The ENG velocity signal was calculated using the two-point central difference method with a spread of two (high frequency noise is suppressed due to the low passed filtering, thus a spread of six, as used for the SSC, is not necessary). Using two electrodes for each eye (one on the temple near the outer canthus of the eye and one nasally) monocular signals were recorded.

The VOG images were sampled interlaced at a sampling frequency of $50 \mathrm{~Hz}$. The VOG signals were interpolated to $1 \mathrm{kHz}$ using the Nyquist sampling theorem [Wierts et al., 2008]. The pupil centre was determined using an improved version of the method as described by Kingma et al. [Kingma et al., 1995]. The VOG velocity signal was calculated using the two-point central difference method with a spread of six. 


\section{Static properties}

The accuracy of each eye measurement technique was estimated by calculating the difference between the absolute eye position and the dot's screen position over all 36 saccades per subject. The sensitivity was estimated to be equal to twice the noise level (average of the standard deviation of each fixation point) during fixation. Saccade amplitudes were calculated from the absolute eye positions. To determine the relations between the saccade amplitudes of ENG and SSC and between VOG and SSC for each eye of each subject, saccade amplitude differences were compared with saccade amplitude averages using the Bland and Altman technique [Bland and Altman, 1986]. Limits of agreement were determined for ENG and SSC and for VOG and SSC.

\section{Dynamic properties}

The relations between saccade peak velocities and saccade amplitudes, the so-called main sequence [Bahill et al., 1975], were determined for each eye of each subject. Exponential curves were fitted through the individual main sequences and were separately fitted for saccades to the left and right [van der Geest and Frens, 2002, Baloh et al., 1975, Houben et al., 2006].

$$
v_{\text {peak }}=C \cdot\left(1-\exp \left(-b \cdot\left|A_{\text {sac }}\right|\right)\right)
$$

with $v_{\text {peak }}$ the saccade peak velocity $\left({ }^{\circ} / \mathrm{s}\right), A_{\text {sac }}$ the saccade amplitude $\left({ }^{\circ}\right), C$ the maximum eye peak velocity for large saccades $(\% / s)$ and $b$ the saturation rate or curve steepness $\left(1 /{ }^{\circ}\right)$.

Statistic analysis was performed using SPSS 17.0 using Wilcoxon signed-rank tests (nonparametric two-related-samples test) and Mann-Whitney $U$ test (non-parametric twoindependent-samples test), both with a threshold for significance of $p=0.05$.

\subsection{Results}

A qualitative comparison of the VOG and ENG signals measured with and without the SSC system in all subjects revealed no magnetic field artifacts in the video images and no artifacts in the twined ENG signals. Moreover, the analyses showed no artifacts in the VOG signals, which might be caused by magnetic interference with the camera electronics or the presence of coils on the eyes, which are visible in the video images. Therefore the results for the static properties are only shown for the measurements 
recorded with the 3 techniques simultaneously (ENG, VOG and SSC). In two subjects the video recordings showed that lens slippage incidently occurs during fast eye movements confirming previous observations by Clarke A.H.

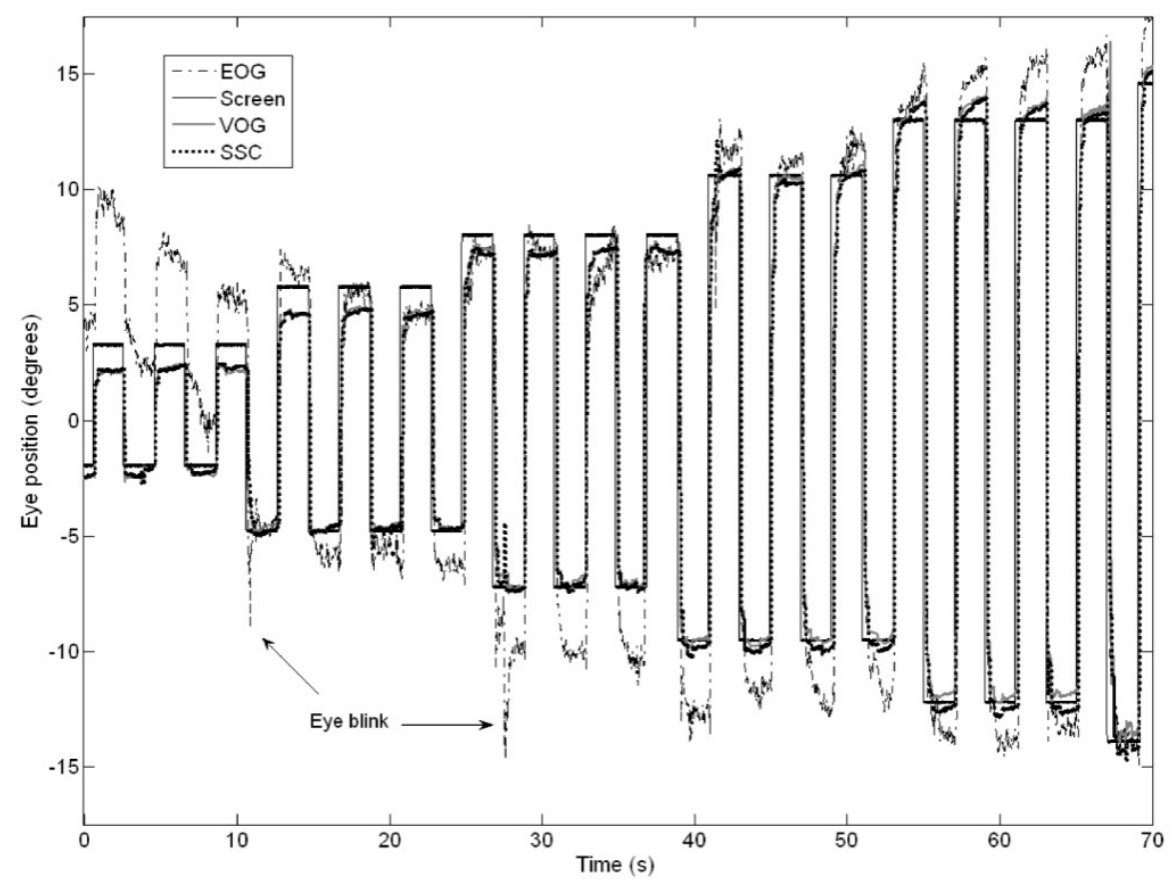

Figure D.3: Position signal for horizontal midline-crossing saccades recorded simultaneously with the ENG, VOG and SSC system. The position of the spot projected on the screen is also shown.

\section{Static properties}

Figure D.3 shows an example of the eye position signal for horizontal midline-crossing saccades recorded simultaneously with the ENG, VOG and SSC system. Based on the three subjects measured, upper values of accuracy and sensitivity of horizontal eye movement measurements in the three different recording techniques are given in table D.1. The accuracy of ENG is worse than $2^{\circ}$, whereas the accuracy of both VOG and SSC is better than $1^{\circ}$. The sensitivity of ENG is better than $1^{\circ}$. The sensitivity of VOG and SSC is better than $0.5^{\circ}$ and $0.2^{\circ}$ respectively. 
Table D.1: Accuracy and sensitivity of horizontal eye movements in three different recording techniques

\begin{tabular}{lll}
\hline Measurement & Accuracy & Sensitivity \\
\hline ENG monocular & $2^{\circ}-15^{\circ}$ & $<1^{\circ}$ \\
VOG monocular & $<1^{\circ}$ & $<0.5^{\circ}$ \\
SSC monocular & $<1^{\circ}$ & $<0.2^{\circ}$ \\
\hline
\end{tabular}

Figure D.4 shows in one subject a typical example of the saccade amplitude differences versus saccade amplitude averages. Limits of agreement for ENG and SSC were $-6^{\circ}$ to $5^{\circ}$ and for VOG and SSC were $-3^{\circ}$ to $3^{\circ}$. In about $70 \%$ of the comparisons there was a clear association between the saccade amplitude differences and averages.

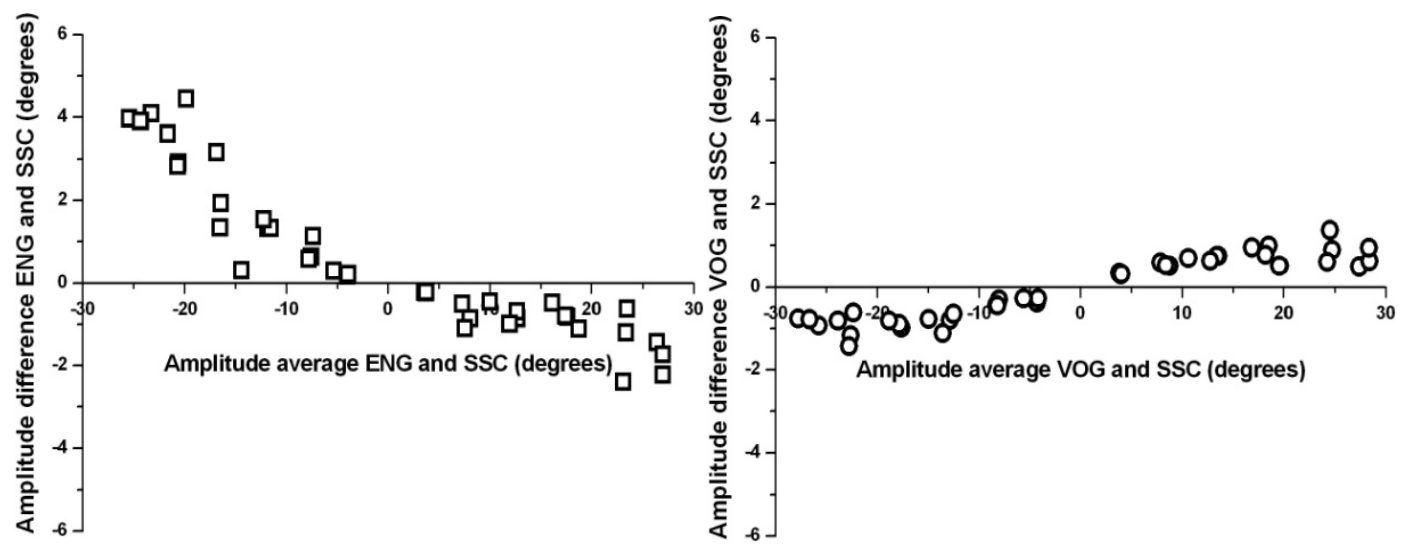

Figure D.4: Typical example in one subject of saccade amplitude differences versus saccade amplitude averages. Left: ENG and SSC, right: VOG and SSC.

\section{Dynamic properties}

In figure D.5 a typical example of the main sequence for the left eye of one subject is presented, showing that the 3 recording techniques ENG, VOG and SSC yield similar results. In table D.2 the average parameters of the exponential curve fits are given, which were separately fitted for saccades to the left and right through the individual main sequences of each eye of each subject and then averaged per subject. Fits were 
made for the measurements while simultaneously recording using the ENG, VOG and SSC system and for the measurements while simultaneously recording using the ENG and VOG. There were no significant differences between the average fit parameters.

More in detail, the VOG fit in figure D.5 shows a significantly higher maximum eye peak velocity $(C=638 \pm 47 \%, b=0.07 \pm 0.01 \%$ ) for saccades to the right (positive amplitudes in figure D.5) than the SSC fit ( $C=553 \pm 29 \%, b=0.08 \pm 0.01 \mu)$. For saccades to the left, the VOG fit $(C=549 \pm 22 \%, b=0.10 \pm 0.01 \%)$ and the SSC fit $(C=$ $544 \pm 15 \%, b=0.11 \pm 0.01 \%$ ) were not significantly different. In table D. 3 the average differences in fit parameters between the 3 recording techniques are given. The average difference between the VOG and SSC fits was significant and systematically positive for $C$ and significantly negative for $b$ for each eye of each subject. Furthermore, the difference between the ENG and SSC fits was significantly positive for $C$ and significantly negative for $b . b$ was also significantly different for the ENG and VOG fits.

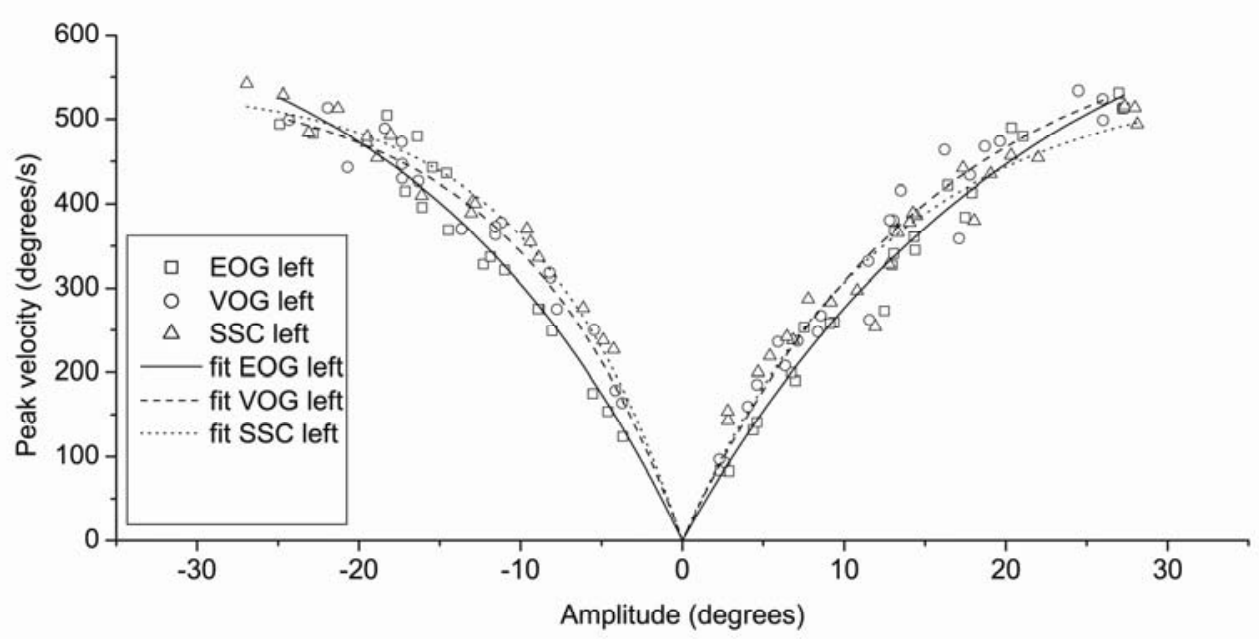

Figure D.5: Main sequence of horizontal midline-crossing saccades of the left eye, simultaneously recorded with the ENG, VOG and SSC method. Positive (negative) saccadic amplitudes correspond to saccades to the right (left). Exponential fits according to equation (1) are also shown. 
Table D.2: Average parameters of the exponential curve fits, separately fitted for saccades to the left and right through the individual main sequences of each eye of each subject

\begin{tabular}{llllll}
\hline Measurement & $\mathbf{C}\left({ }^{\circ} / \mathbf{s}\right)$ & $\mathbf{b}\left(/^{\circ}\right)$ & Measurement & $\mathbf{C}\left({ }^{\circ} / \mathbf{s}\right)$ & $\mathbf{b}\left(/^{\circ}\right)$ \\
ENG, VOG, SSC & & \multicolumn{5}{c}{ ENG, VOG } \\
\hline ENG & $527 \pm 135$ & $0.09 \pm 0.03$ & ENG & $591 \pm 119$ & $0.08 \pm 0.03$ \\
VOG & $487 \pm 87$ & $0.11 \pm 0.03$ & VOG & $535 \pm 64$ & $0.11 \pm 0.03$ \\
SSC & $442 \pm 79$ & $0.12 \pm 0.03$ & & & \\
\hline
\end{tabular}

Table D.3: Average differences in parameters of the exponential curve fits while simultaneously recording with the ENG, VOG and SSC system. * marks significant differences

\begin{tabular}{lll}
\hline Difference between & $\mathbf{C}\left({ }^{\circ} / \mathbf{s}\right)$ & $\mathbf{b}\left(/^{\circ}\right)$ \\
\hline VOG - SSC & $56(4 \text { to } 112)^{*}$ & $-0.02(-0.05 \text { to } 0.02)^{*}$ \\
ENG - SSC & $86(-39$ to 177$) *$ & $-0.04(-0.09 \text { to } 0)^{*}$ \\
ENG - VOG & $53(-113$ to 139$)$ & $-0.03(-0.10 \text { to } 0.03)^{*}$ \\
\hline
\end{tabular}

Although not significant, table D.2 shows higher maximum eye peak velocities $C$ while recording without the SSC system for the ENG (591 $\pm 119 \%$ vs. $527 \pm 135 \%$ s) and VOG ( $535 \pm 64 \%$ vs. $487 \pm 87 \%$ s) measurements as compared to the measurements while simultaneously recording with the SSC system. In table D.4 the average differences in $C$ between the measurements while recording without and with the SSC system for each eye of each subject are given. ENG and VOG both had significantly higher maximum eye peak velocities $C$ in the measurements without the SSC system as compared to the measurements while simultaneously recording with the SSC system. 
Table D.4: Average differences in parameters of the exponential curve fits without the SSC system as compared to with the SSC system. * marks significant differences

\section{$\mathrm{C}\left({ }^{\circ} / \mathrm{s}\right) \quad \mathrm{b}\left(/^{\circ}\right)$}

$\begin{array}{lll}\text { ENG } & 55(-68 \text { to } 196) * & 0.00(-0.05 \text { to } 0.05) \\ \text { VOG } \quad 48(-47 \text { to } 151) * & 0.00(-0.05 \text { to } 0.02)\end{array}$

\subsection{Discussion}

\section{Static properties}

The drift in the ENG signal was about $0.25 \%$, which is no problem for the accurate determination of saccade amplitudes, since the duration of a relatively large amplitude saccade of the order of $40^{\circ}$ does not exceed about $100 \mathrm{~ms}$ [Carpenter, 1988]. But the ENG drift is a common problem for determination of absolute eye position. Therefore ENG had a much lower accuracy than VOG and SSC. The sensitivity of VOG and SSC recordings was also better than ENG, whereas SSC had an even better sensitivity than VOG, probably because different eye features such as a dark iris or the upper eye lid covering the pupil can deteriorate the video signal analysis of the VOG system. The limits of agreement between ENG and SSC were twice as large as the limits of agreement between VOG and SSC, showing that VOG is better than ENG for determination of saccadic amplitudes. Thus, not to our surprise, VOG and SSC are the preferred techniques to measure eye saccadic amplitudes.

\section{Dynamic properties}

The 3 recording techniques ENG, VOG and SSC yield similar results for the main sequence of horizontal midline-crossing saccades, again indicating that online real-time $50 \mathrm{~Hz}$ VOG using the Nyquist sampling theorem to interpolate the data to $1 \mathrm{kHz}$ is appropriate for detecting saccade peak velocities [Wierts et al., 2008]. However, an important feature noticed was the fact that VOG and ENG had significantly higher individual peak velocities than SSC. In other words, when averaged, the differences in peak velocities were not significant for the different eye recording techniques, whereas for the individual case (each subject and each eye) systematic differences were 
observed. This phenomenon was caused by the inter-subject variation which was larger than the intra-subject variation. Since the main sequences were obtained from saccades simultaneously recorded with ENG, VOG and SSC, the observed difference could be caused by either technique. Observation of our video recordings shows that the search coil is not really fixed on the eye, confirming previous observations by Clarke A.H. Moreover, underestimation of saccade peak velocity of the SSC technique was also observed in other studies in which a direct comparison was made between the values of the peak velocity of saccades simultaneously recorded with both VOG and SSC [van der Geest and Frens, 2002, Houben et al., 2006, DiScenna et al., 1995]. Therefore, this phenomenon is a result of lens slippage of the search coil and suggests that VOG and ENG yield the correct values. Instability of the head-mounted eye tracker, as mentioned by Houben and colleagues [Houben et al., 2006] is very unlikely to be the cause, because ENG also had significantly higher peak velocities than SSC. The phenomenon cannot be ascribed to the effect of the scleral search coil on the kinematics of the eye [DiScenna et al., 1995, Frens and van der Geest, 2002] since the saccades were simultaneously measured with both the VOG and SSC method. However, the coil does have an effect on the eye dynamics because both ENG and VOG have higher maximum eye peak velocities in the measurements without a search coil on the eye as compared to with a coil on the eye. Because no magnetic field artifacts in the video images or in the ENG signals were present, the lower peak velocities cannot be due to the SSC system but have to be due to the coils on the eyes. Thus, VOG and ENG are the preferred techniques to measure eye peak velocities.

We therefore conclude that, based on the static and dynamic properties comparison, VOG is the favoured technique to measure eye movements. Unfortunately, clinical practice taught us that VOG sometimes fails to detect the pupil centre correctly without being noticed by the technician, for example in subjects with slant-eyes, large pupils or in examinations in which the pupil makes large vertical excursions. We observed this problem for example when we monitored eye movements with VOG and ENG simultaneously in Gilles de la Tourette patients provided with a deep brain stimulator. Here the ENG is often contaminated by the electrical activity from the stimulator, which forced us to use the VOG as well. We however noticed that in 3 out of the 9 patients VOG was inaccurate to detect the pupil properly because of slant eyes or problems with keeping the eyes open for sufficiently long time during testing. In such cases, - without warning or notice - no reliable eye position signal will be obtained from the video 
eyetracker, whereas the ENG method can still record eye movements, even when the eyes are completely closed. In our recent pilot study with simultaneous use of ENG and VOG, it was observed that VOG failed in a detecting the correct eye position in about $17 \%$ of the subjects $(n=112)$. We recently developed techniques to overcome this problem by implementing algorithms that warn the technician for failures in accurate pupil detection [Jansen et al., 2009]. This observation is a clear argument in favor of application of ENG at least as a backup technique. The SSC technique is not suited for clinical practice, because of patient discomfort leading to restricted measurement time and the unsuitability for routine evaluations. With stabilized and frequently calibrated ENG signals, the sensitivity of the ENG technique is in general acceptable.

Additionally, the SSC technique, that is often mentioned to be the gold standard of eye movement recordings, appears to suffer from at least 2 limitations: 1) lens slippage and 2) reduction of eye peak velocities by the fixation of lenses on the cornea, resulting in underestimation of the real eye peak velocities in pure physiological conditions. However, these effects are small and only relevant for large amplitude saccades. The SSC technique is suitable for measuring more static properties of the eye, like saccade amplitudes, because of its superior time and spatial resolution. 


\subsection{Conclusions}

Based on the static properties VOG and SSC are the preferred techniques to measure eye saccadic amplitudes. ENG is less appropriate because of the substantial drift in the ENG signal. Also, due to non-linearities in ENG it is not accurate in detection of monocular eye movements; in contrast ENG is more accurate in movement detection of the average of both eyes. Based on the dynamic properties VOG and ENG are the preferred techniques to measure eye peak velocities because of lens slippage of the search coil and slowing down of the eye dynamics by the presence of the search coil on the eye.

In other words, based on both the static and dynamic properties, from a technical point of view online real-time $50 \mathrm{~Hz}$ VOG using the Nyquist sampling theorem to interpolate the data to $1 \mathrm{kHz}$ appears to be the preferred technique for measurement of eye saccades with amplitudes larger than $5^{\circ}$ [Wierts et al., 2008]. Even more important is the fact that a sampling rate of $50 \mathrm{~Hz}$ is appropriate for detecting the clinical relevant saccade peak velocities [Wierts et al., 2008]. Moreover, the physician is mainly interested in the slow phase velocity, as it is the main clinical relevant output measure of the vestibular ocular reflex. Since the dominant frequency of this slow phase velocity is in the low frequency domain $(0.025 \mathrm{~Hz}$ for the caloric test), a sampling frequency of 50 Hz suffices. 


\section{References}

Allum, J. H., Honegger, F. \& Troescher, M. (1998) Principles underlying real-time nystagmus analysis of horizontal and vertical eye movements recorded with electro-, infra-red-, or video-oculographic techniques. J Vestib Res, 8, 449-63.

Bahill, A. T., Clark, M. R. \& Stark, L. (1975) The main sequence, a tool for studying human eye movements. Math Biosci., 24, 191-197.

Bahill, A. T., Kallman, J. S. \& Lieberman, J. E. (1982) Frequency limitations of the two-point central difference differentiation algorithm. Biol Cybern, 45, 1-4.

Baloh, R. W., Sills, A. W., Kumley, W. E. \& Honrubia, V. (1975) Quantitative measurement of saccade amplitude, duration, and velocity. Neurology, 25, 1065-70.

Bland, J. M. \& Altman, D. G. (1986) Statistical methods for assessing agreement between two methods of clinical measurement. Lancet, 1, 307-10.

Carpenter, R. H. S. (1988) Movement of the eyes, Pion Limited.

Clarke, A. H., Ditterich, J., Druen, K., Schonfeld, U. \& Steineke, C. (2002) Using high frame rate CMOS sensors for three-dimensional eye tracking. Behav Res Methods Instrum Comput, 34, 549-60.

Collewijn, H., Van Der Mark, F. \& Jansen, T. C. (1975) Precise recording of human eye movements. Vision Res, 15, 447-50.

Discenna, A. O., Das, V., Zivotofsky, A. Z., Seidman, S. H. \& Leigh, R. J. (1995) Evaluation of a video tracking device for measurement of horizontal and vertical eye rotations during locomotion. J Neurosci Methods, 58, 89-94.

Frens, M. A. \& Van Der Geest, J. N. (2002) Scleral search coils influence saccade dynamics. J Neurophysiol, 88, 692-8.

Harris, C. M., Wallman, J. \& Scudder, C. A. (1990) Fourier analysis of saccades in monkeys and humans. J Neurophysiol, 63, 877-86.

Houben, M. M., Goumans, J. \& Van Der Steen, J. (2006) Recording three-dimensional eye movements: scleral search coils versus video oculography. Invest Ophthalmol Vis Sci, 47, 179-87.

Jansen, S. M. H., Kingma, H. \& Peeters, R. L. M. (2009) A confidence measure for real-time eye movement detection in video-oculography, IFBM, 23, 335-39

Juhola, M., Jantti, V. \& Pyykko, I. (1985) Effect of sampling frequencies on computation of the maximum velocity of saccadic eye movements. Biol. Cybern., 53, 67-72.

Kessler, P., Zarandy, M. M., Hajioff, D., Tomlinson, D., Ranalli, P. \& Rutka, J. (2008) The clinical utility of search coil horizontal 118estibule-ocular reflex testing. Acta Otolaryngol, 128, 29-37.

Kingma, H., Gullikers, H., De Jong, I., Jongen, R., Dolmans, M. \& Stegeman, P. (1995) Real time binocular detection of horizontal vertical and torsional eye movements by an infra red video-eye tracker. Acta Otolaryngol Suppl, 520 Pt 1, 9-15.

Kumar, A. \& Krol, G. (1992) Binocular infrared oculography. Laryngoscope, 102, 367-78.

North, A. W. (1965) Accuracy and precision of electro-oculographic recording. Invest Ophthalmol, 4, 343-8. 
Press, W. H., Teukolsky, S. A., Vetterling, W. T. \& Flannery, B. P. (1995) Numerical Recipes in C, Press Syndicate of the University of Cambridge.

Reulen, J. P., Marcus, J. T., Koops, D., De Vries, F. R., Tiesinga, G., Boshuizen, K. \& Bos, J. E. (1988) Precise recording of eye movement: the IRIS technique. Part 1. Med Biol Eng Comput, 26, 20-6.

Robinson, D. A. (1963) A method of measuring eye movement using a scleral search coil in a magnetic field IEEE Trans Biomed Eng, 10, 137-45.

Schmid-Priscoveanu, A. \& Allum, J. H. (1999) Infrared and video oculography - alternatives to electrooculography? HNO, 47, 472-8.

Truong, D. M. \& Feldon, S. E. (1987) Sources of 119 estibul in infrared recording of eye movement. Invest Ophthalmol Vis Sci, 28, 1018-22.

Usui, S. \& Amidror, I. (1982) Digital low-pass differentiation for biological signal processing. IEEE Trans Biomed Eng, 29, 686-93.

Van Der Geest, J. N. \& Frens, M. A. (2002) Recording eye movements with video-oculography and scleral search coils: a direct comparison of two methods. J Neurosci Methods, 114, 18595.

Wierts, R., Janssen, M. J. \& Kingma, H. (2008) Measuring saccade peak velocity using a lowfrequency sampling rate of $50 \mathrm{~Hz}$. IEEE Trans Biomed Eng, 55, 2840-2.

Wong, A. M., Mcreelis, K. \& Sharpe, J. A. (2006) Saccade dynamics in peripheral vs central sixth nerve palsies. Neurology, 66, 1390-8.

Young, L. R. \& Sheena, D. (1975) Survey of eye movement recording methods. Behavior Research Methods \& Instrumentation, 7, 397-429. 


\section{Part E}

SUMMARYAND CONCLUDING REMARKS 
Part E

This part would not have been possible without the discussions with Nils Guinand, Raymond van de Berg and Herman Kingma. 
The aim of this thesis was to evaluate and improve existing and develop advanced diagnostic and therapeutic possibilities for vestibular medicine, based on a theoretical background as presented in part $\mathbf{A}$.

The ambulatory vibrotactile biofeedback (AVBF) system is introduced in part B and its effects on orientation, stance and gait were explored. The purpose of the AVBF system is to improve postural control in vestibular patients by vibrotactile biofeedback of tilt of head or trunk. It was first demonstrated in chapter 1 that the AVBF system has the ability to change earth vertical orientation even in healthy subjects, which had to reorient a tilting platform back to absolute horizontal using a velocity controlled joystick. Secondly, in chapter $\mathbf{2}$, in patients with severe bilateral vestibular hyporeflexia the AVBF system was used to increase postural stability during gait. Observational gait analysis was used to score individual balance during gait and it was shown that in general the AVBF system significantly improves balance during gait. However, we were able to identify significant individual improvements of balance during gait in only 2 patients with the AVBF system activated. In the majority of our patients it increased confidence, independence and a feeling of balance and safety.

Finally, in chapter $\mathbf{3}$, in another group of patients with severe bilateral vestibular losses a placebo-controlled study was performed to evaluate the effect of the AVBF system on body sway in stance. In $40 \%$ of the patients body sway decreased significantly using biofeedback. This improvement with true biofeedback was however only observed when an improvement was present with placebo biofeedback as well. The improvement in body sway was, at least partially, caused by other effects than biofeedback like training, increased self confidence or alertness. Additionally, training to optimise use of the AVBF system seems to be essential, because improvement of balance by biofeedback is only shown in the minority of patients with balance problems. Adaptation effects do however occur and continuous use of the AVBF system might not be necessary. The feasibility of the AVBF system for vestibular rehabilitation and balance improvement in stance and gait is however strongly supported by our work.

In part $\mathbf{C}$ a new diagnostic possibility -perception thresholds for rotation, tilt and translation - was explored. For perception of rotation, deceleration step stimuli during continuous subthreshold acceleration rotation were applied in chapter 4 as a new motion paradigm to induce specific vestibular rotation perceptions opposite to the actual rotation direction. Non-vestibular cues for rotation were masked. Subjects were 
instructed to verbally indicate a change in rotation direction. The normal range of perception threshold for rotation was up to $6 \%$. The perception threshold for rotation in patients with a history pointing to a possible vestibular deficit was increased in especially those patients with the most severe pareses based on the classical tests. However, $72 \%$ of our patients with a partial or complete unilateral or bilateral hypofunction of the horizontal canals had normal rotation perception thresholds. It might be that their vertical canals and otolith function enabled the rotation perception, or that vestibular losses have a specific frequency dependence. Together with the fact that different vestibular diagnostic tests have differing frequency contents, it might be that the perception threshold for rotation has a complimentary value in diagnostics.

For perception of tilt and translation, seated subjects were submitted to 1) unilateral and bilateral eccentric rotations (centrifugation), 2) whole body translatory decelerations opposite to the movement direction on a linear sled and 3) discrete slow velocity platform tilts in chapter $\mathbf{5}$. Tactile, semicircular canal, visual and auditory cues were masked. Subjects were instructed to verbally indicate the perceived direction of tilt or translation. The normal range of perception threshold for tilt during centrifugation was up $1.9-5.6^{\circ}$, which to our interpretation mainly depends on otolithic function. Tilt perception during whole body decelerations and discrete platform tilts mainly depended on somatosensory information, showing the dominant role of the somatosensory system for the perception of body orientation in these conditions. The perception threshold for tilt during eccentric rotations thus seems to be the most promising perception tool for the evaluation of utricular function.

In part $\mathbf{D}$ we focused on improvement of infrared video oculography (VOG), since about 10 years a quite popular diagnostic method to visualize and quantify eye movements for testing the oculomotor and vestibular system. Real-time systems with a low sampling frequency of $50 / 60 \mathrm{~Hz}$ are used for clinical practice, but are generally considered not to be suited for measuring fast eye movements or saccadic peak velocities, because high sampling frequencies are in literature always mentioned to be necessary. In chapter 6 it was shown that without any additional postprocessing, VOG can indeed largely underestimate saccade peak eye velocities. Saccadic eye movements, having an amplitude of at least $5^{\circ}$, are however in good approximation bandwidth limited up to a frequency of $25-30 \mathrm{~Hz}$. Therefore the Nyquist theorem states that 1 ) a sampling of 50-60 $\mathrm{Hz}$ is sufficiently high to avoid aliasing and 2) a signal at a higher temporal resolution can be reconstructed. By interpolating the data to $1 \mathrm{kHz}$ it was shown that deviations of 
saccade peak velocities were on average $3 \%$, much smaller than normal variations. VOG of $50-60 \mathrm{~Hz}$ using the Nyquist sampling theorem to interpolate the data to $1 \mathrm{kHz}$ is thus appropriate for determining saccade peak velocities for basic clinical application.

Additionally, in chapter 7,1 ) the semi-invasive scleral search coil technique (SSC), considered to be the gold standard for eye movement measurements, 2) the regular clinically applied electro nystagmography (ENG) method and 3) a $50 \mathrm{~Hz}$ VOG system using the Nyquist sampling theorem to interpolate the data to $1 \mathrm{kHz}$ were compared. It was shown that for detection of eye saccadic amplitudes, VOG and SSC are the preferred techniques, because substantial drift makes ENG less appropriate. Also, due to nonlinearities in ENG it is not accurate in detection of monocular eye movements; in contrast ENG is more accurate in movement detection of the average of both eyes. VOG and ENG are the preferred techniques to measure eye peak velocities, because of lens slippage of the search coil and slowing down of the eye dynamics by the presence of the search coil on the eye. Thus, VOG of $50-60 \mathrm{~Hz}$ using the Nyquist sampling theorem to interpolate the data to $1 \mathrm{kHz}$ appears to be the preferred clinical technique for measurement of eye movements, from a technical point of view. However, in subjects with slant eyes or problems with keeping their eyes open during testing, VOG fails to detect the pupil centre correctly and most frequently used VOG systems do not warn the technician for the errors made. The ENG method is much more robust and can despite some other draw backs - record eye movements in almost any condition, which argues for clinical application of ENG, at least as a backup technique. 


\section{Concluding remarks}

When somatosensory information is present and not distorted, it is a very prominent cue for tilt perception and strongly interacts with vestibular input [part C, chapter 5, Horak, 2010]. This explains why a large amount of people with severe vestibular losses do not complain of balance problems, but which also opens the possibility for sensory substitution in those who do have severe balance problems [part B, chapters 2 and 3]. Based on its physical properties, the otolith system might have the highest sensitivity for low frequency tilts (gravity and constant linear accelerations) [Melvill Jones, 1979]:

- we investigated the perception of tilt during centrifugation [part C, chapter 5]. It was shown that perception of tilt occurs at tilt angle of minimal $2{ }^{\circ}$ which is quite inaccurate for postural control because the limit of stability in healthy subjects is about $6^{\circ}$ [Nashner et al., 1989].

- we equipped the AVBF system [part B] with linear accelerometers. It was shown that the AVBF system significantly improves stance, gait and confidence in the majority of our patients with severe bilateral vestibular losses and frequent falls, but improvements in stance and gait were only present in the minority of patients and improvements with true biofeedback were only observed where an improvement was present with placebo biofeedback as well.

This could imply that it might be possible to control balance without otolith system and solely with our visual and somatosensory system, especially because the time constant for detection of linear accelerations and thus tilts is about 1 second [part A]. Additionally, it has been shown that 1 ) for complete static posture the availability of otolith inputs does not imply a better perception of body orientation [Bringoux et al., 2003] and 2) vestibular nucleus activity is modulated during tilt in animals following definitive ablation of vestibular inputs [Yates et al., 2000]. However:

- it has been shown that different mechanisms contribute to postural control and postural perception [Merfeld et al., 2005, Mittelstaedt, 1999] and that it is likely that processing at a subconscious level occurs before conscious perception [Teasdale et al., 1999]. As we all know, balance control is in general not a conscious process. It was therefore suggested that at a perceptual level the otolith organs cannot be considered as efficient detectors of gravity in quasistatic conditions [Teasdale et al., 1999], in the absence of all other spatial 
information [Mittelstaedt, 1999]. This is in certain situations (for example when trying to escape when buried by a snow avalanche or from a sinking car in troubled waters) very problematic. Additionally, our results on perception thresholds for rotation [part C, chapter 4] also indicate that reflex measurements might have a higher sensitivity than perception tests.

- the AVBF system feeds back the absolute orientation of the gravity vector, although the accuracy of body orientation perception is very poor [part $C$, chapter 5, part B, chapter 1].

So we advise that the future AVBF system should indicate tilt angle velocity instead of tilt angle, for which gyroscopes are also better suited. Recently it was however suggested that the impact of feedback of tilt angle versus tilt angle velocity does not differ, but that the combination of both works best to decrease body sway [Wall and Kentala, 2010], similar to functional vestibular organs in which the semicircular canals support the otolith organs to discriminate between translations and tilts [part A].

As the vestibular system is situated in the head, vestibular information is a top-down vertical reference and thus body posture requires integration and interpretation with somatosensory information, particularly from the neck [part A, Horak, 2010]. A robust model of sensory integration for postural control suggests that the nervous system is always comparing somatosensory with vestibular information and when the two systems agree, somatosensory is primarily relied upon [Mergner et al., 2002]. With disagreement between somatosensory and vestibular information, the nervous system depends more and more on labyrinthine input [part A]. In this way the vestibular system is a strongly learning and adaptive system [part A] and vestibular information provides a 'set point' for postural somatosensory feedback loops [Horak, 2010], which can be problematic for patients with severe vestibular losses. Biofeedback of this 'set point' absolute orientation of the head and trunk in space - as well as body tilt movements, discriminated from translatory movements, can only be accomplished if the future AVBF system is able to indicate both tilt angle and tilt rate and should be equipped with both accelerometers and gyroscopes, just as a functional vestibular organ.

The future AVBF system should be developed and evaluated for 1. a broader clinical application, 2. performance in daily life and 3. quality of life of patients. It will at least have a benefit in patient motivation to be more active, to support training and to learn more about postural sway and its dependence on daily life activities. Moreover, because 
there is a need for training and rehabilitation, biofeedback is a rewarding approach as it gives a positive feeling if no feedback has been given during a task. Therefore a custom made, easy to use, AVBF system is currently under development in our clinic in cooperation with Maastricht Instruments. The artist impression below shows one of the numerous ideas we have for several exciting challenges ahead.

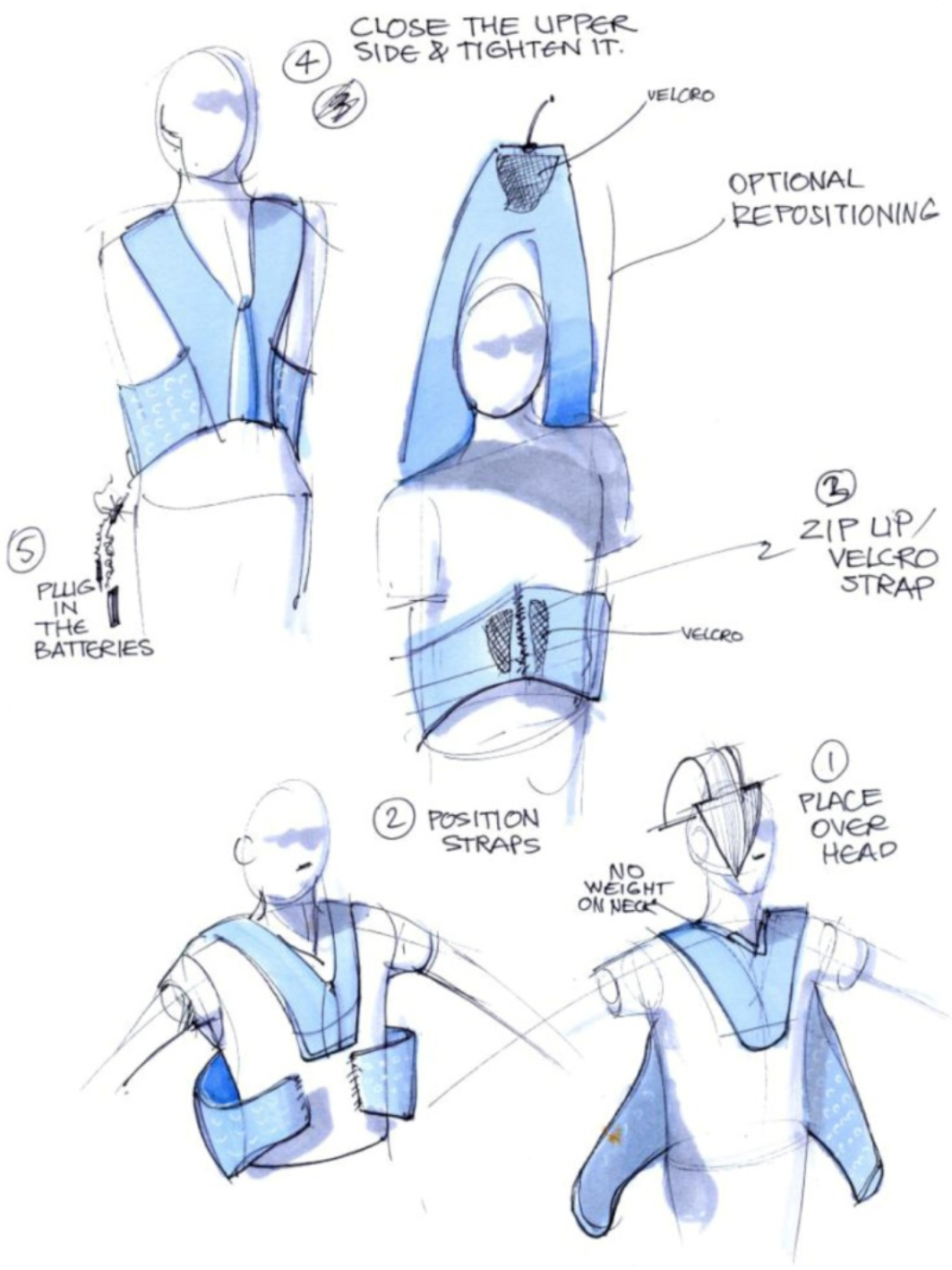

Artist impression of the future AVBF system, courtesy of Herman Assink 
Rationing in health care is a major challenge, therefore patients need to be selected for specific rehabilitation programs or advanced technological therapeutic possibilities like the AVBF system or even a vestibular implant. The latter is in cooperation with worldwide leading groups under research in our clinic. To be able to select a limited number of patients, perception tests will play an important role in the diagnosis and treatment of patients [part $\mathrm{C}$ ], because perception measurements in general seem to have a closer correlation with specific complaints than reflex measurements. There is however a strong interaction between and individual dependence on vestibular and somatosensory information [part A], which also explains the low sensitivity of vestibular perception tests explored in this thesis. Moreover, because we tried to mask as much as possible all non-vestibular cues, unfamiliar stimuli are presented which might explain a large variability. So probably learning will play a prominent role. This is currently all under research in our clinic. Additionally it can be expected that vestibular tests with differing frequency characteristics all are complimentary in showing vestibular deficits, which might have specific frequency dependence. 


\section{References}

Bringoux, L., Nougier, V., Barraud, P. A., Marin, L. \& Raphel, C. (2003) Contribution of somesthetic information to the perception of body orientation in the pitch dimension. $Q J$ Exp Psychol A, 56, 909-23.

Horak, F. B. (2010) Postural compensation for vestibular loss and implications for rehabilitation. Restor Neurol Neurosci, 28, 57-68.

Melvill Jones, G. (1979) Biophysics of the Peripheral End Organs. IN WILSON, V. J. \& MELVILLJONES, G. (Eds.) Mammalian vestibular physiology. New York, Plenum Press.

Merfeld, D. M., Park, S., Gianna-Poulin, C., Black, F. O. \& Wood, S. (2005) Vestibular perception and action employ qualitatively different mechanisms. I. Frequency response of VOR and perceptual responses during Translation and Tilt. J Neurophysiol, 94, 186-98.

Mergner, T., Maurer, C. \& Peterka, R. J. (2002) Sensory contributions to the control of stance: a posture control model. Adv Exp Med Biol, 508, 147-52.

Mittelstaedt, H. (1999) The role of the otoliths in perception of the vertical and in path integration. Ann N Y Acad Sci, 871, 334-44.

Nashner, L. M., Shupert, C. L., Horak, F. B. \& Black, F. O. (1989) Organization of posture controls: an analysis of sensory and mechanical constraints. Prog Brain Res, 80, 411-8; discussion 395-7.

Teasdale, N., Nougier, V., Barraud, P. A., Bourdin, C., Debu, B., Poquin, D. \& Raphel, C. (1999) Contribution of ankle, knee, and hip joints to the perception threshold for support surface rotation. Percept Psychophys, 61, 615-24.

Wall, C., $3^{\text {rd }}$ \& Kentala, E. (2010) Effect of displacement, velocity, and combined vibrotactile tilt feedback on postural control of vestibulopathic subjects. J Vestib Res, 20, 61-69.

Yates, B. J., Jian, B. J., Cotter, L. A. \& Cass, S. P. (2000) Responses of vestibular nucleus neurons to tilt following chronic bilateral removal of vestibular inputs. Exp Brain Res, 130, 151-8. 


\section{Scientific Output}

Janssen M, Lauvenberg M, van der Ven W, Bloebaum T and Kingma H. (2011) Perception Threshold for Tilt. Otol Neurotol.

Janssen M, Pas R, Aarts J, Janssen-Potten $\mathrm{Y}$, Vles H, Nabuurs C, van Lummel R, Stokroos $R$ and Kingma H. (2011) Clinical observational gait analysis to evaluate improvement of balance during gait with vibrotactile biofeedback. Phys Res Int.

Janssen M, Stokroos, R., Aarts, J, Van Lummel, R and Kingma, H (2010) Salient and placebo vibrotactile feedback are equally effective in reducing sway in bilateral vestibular loss patients. Gait Posture, 31, 213-7.

Wierts R, Janssen $\mathbf{M}$ and Kingma $H$ (2008) Measuring saccade peak velocity using a lowfrequency sampling rate of $50 \mathrm{~Hz}$. IEEE Trans Biomed Eng, 55, 2840-2.

Janssen M, van der Ven W, Wolters M and Kingma H. (2011) Clinical application of perception threshold of horizontal plane rotation. J Vest Res, submitted. 
Janssen M, Stokroos R and Kingma H (2007) Het Vibrotactiel Labyrinthair Substitutie systeem (VLS): klinische evaluatie van een ambulante prothese voor patiënten met ernstig vestibulair functieverlies. Nederlandse Tijdschrift voor KNOheelkunde, 13 (4), 177-84

Janssen M, Pas R, Vles $\mathrm{H}$, Janssen-Potten $\mathrm{Y}$, Aarts J, van Pinxteren S, Stokroos R and Kingma H (2005) De VLS, een kunstmatig evenwichtsorgaan. Technologie in de gezondheidszorg, 10 en 11, oktober en november 2005

Pas R, Janssen M, Aarts J, Nabuurs C, van Pinxteren S, Janssen-Potten Y, Stokroos R and Kingma $H$ (2004) De SsLsS, een kunstmatig evenwichtsorgaan. Klinische Fysica, $3+4$

van Pul C, Buijs J, Janssen M, Roos G, Vlaardingerbroek M and Wijn P (2005) Selecting the best index to follow the temporal evolution of $A D C$ and diffusion anisotropy after hypoxic-ischemic white matter injury in neonates. American Journal of Neuroradiology, 26(3), 469-81

Janssen M and Duynstee H (2004) Het elektronisch patientendossier en privacy. Klinische Fysica, 3+4 


\section{Samenvatting}

Het doel van dit proefschrift was het evalueren en verbeteren van bestaande en ontwikkelen van geavanceerde diagnostische en therapeutische mogelijkheden voor de vestibulaire geneeskunde, gebaseerd op een theoretische basis (deel A).

Het vibrotactile labyrinthair substitutie (VLS) system is geïntroduceerd in deel B en de effecten van de VLS op oriëntatie, houding en gangbeeld werden verkend. Het doel van het VLS systeem is om houdingsevenwicht in vestibulaire patiënten te verbeteren d.m.v. vibrotactiele biofeedback van kanteling van hoofd of romp. Allereerst werd aangetoond in hoofdstuk 1 dat het VLS systeem in gezonde proefpersonen, die een gekanteld platform m.b.v. een snelheidsgestuurde joystick horizontaal moesten zetten, oriëntatie t.o.v. de verticaal kan beïnvloeden. Vervolgens werd, in hoofdstuk 2, het VLS systeem gebruikt om in patiënten met een ernstige tweezijdige vestibulaire hyporeflexie houdingsevenwicht tijdens lopen te verbeteren. Observationele gangbeeld analyse werd gebruikt om individuele balans tijdens lopen te scoren en er werd aangetoond dat het VLS systeem in het algemeen een significante verbetering van balans tijdens lopen teweegbrengt. Echter, we konden in slechts 2 patiënten een significante individuele verbetering van balans tijdens lopen identificeren. Toch had het VLS systeem in de meerderheid van de patiënten meer zelfvertrouwen, onafhankelijkheid en een gevoel van balans en veiligheid tot gevolg.

Tenslotte werd, in hoofdstuk 3, in een andere groep patiënten met ernstige tweezijdige vestibulaire verliezen, een placebo gecontroleerde studie uitgevoerd om het effect van de VLS op houdingsevenwicht tijdens stilstaan te evalueren. In $40 \%$ van de patiënten nam het houdingsevenwicht m.b.v. biofeedback significant toe. Deze verbetering was echter even groot met placebo biofeedback als met echte biofeedback. Deze verbetering was dus, in ieder geval deels, veroorzaakt door andere effecten dan de biofeedback van het VLS systeem, zoals training, meer zelfvertrouwen of alertheid. Additioneel, training om het gebruik van het VLS systeem te optimaliseren lijkt essentieel omdat verbetering van houdingsevenwicht m.b.v. biofeedback slechts in de minderheid van de patiënten met balans problemen wordt waargenomen. Adaptatie effecten treden ook op, dus een continu gebruik van het VLS systeem is wellicht niet noodzakelijk. Maar de mogelijkheden van het VLS systeem voor vestibulaire rehabilitatie en verbetering van balans worden sterk ondersteund door ons werk. 
In deel C werd een nieuwe diagnostische mogelijkheid - perceptie drempels van rotatie, kanteling en translatie - verkend. Voor perceptie van rotatie is een nieuw bewegingsparadigma met vertragingsstimuli gedurende continue subthreshold versnelling toegepast in hoofdstuk 4 , om specifieke vestibulaire rotatie percepties tegengesteld aan de daadwerkelijke draairichting op te wekken. Niet-vestibulaire aanwijzingen voor rotatie werden gemaskeerd. Proefpersonen werden geïnstrueerd om verbaal de verandering in draairichting aan te geven. Normaalwaarden van rotatie perceptie drempels waren tot $6 \%$. De perceptie drempel voor rotatie in patiënten met een geschiedenis indicatief voor een mogelijke vestibulaire dysfunctie was verhoogd in voornamelijk die patiënten met de meest ernstige pareses gebaseerd op de klassieke testen. Echter, $72 \%$ van onze patiënten met een gedeeltelijke of volledige unilaterale of tweezijdige hypofunctie van de horizontale kanalen hadden een normale rotatie perceptie drempels. Het zou kunnen dat hun verticale kanalen en otolieten functie rotatie perceptie mogelijk maakten, of dat vestibulaire verliezen een specifieke frequentie afhankelijkheid hebben. In combinatie met het feit dat verschillende vestibulaire diagnostische testen een verschillende frequentie inhoud hebben, zou het kunnen dat rotatie perceptie drempels een complementaire diagnostische waarde hebben.

Voor perceptie van kanteling en translatie, werden zittende proefpersonen onderworpen aan 1) een- en tweezijdige excentrische rotaties (centrifugatie), 2) translatie vertragingen tegengesteld aan de bewegingsrichting van een lineaire slede en 3) langzame snelheid discrete platform kantelingen in hoofdstuk 5. Tactiele, halfcirkelvormige kanalen, visuele en auditieve aanwijzingen werden gemaskeerd. Proefpersonen werden geïnstrueerd om verbaal de waargenomen richting van kanteling of translate aan te geven. Normaalwaarden van kantel perceptie drempels gedurende centrifugatie waren $1.9-5.6^{\circ}$, welke naar onze mening vooral afhankelijk is van de otolieten functie. Kantel perceptie gedurende translatie vertragingen en discrete platform kantelingen werd vooral bepaald door somatosensorische informatie, wat de dominante rol van het somatosensorische systeem voor de perceptie van lichaamsoriëntatie aantoont in deze condities. De perceptie drempel voor tilt gedurende excentrische rotaties lijkt dus de meest veelbelovende methode voor de perceptieve evaluatie van utriculaire functie.

In deel D werd gefocusseerd op verbetering van infrarode video oculografie (VOG), een sinds ongeveer 10 jaar redelijk populaire diagnostische methode om oogbewegingen te 
visualiseren en kwantificeren bij de evaluatie van het oculomotor en vestibulaire systeem. Real-time systemen met een lage bemonsterfrequentie van $50 / 60 \mathrm{~Hz}$ worden gebruikt in de klinische praktijk, maar worden in het algemeen niet geschikt geacht om snelle oogbewegingen of saccade piek snelheden te meten, omdat in de literatuur hoge bemonsterfrequenties altijd als noodzakelijk worden geacht. In hoofdstuk 6 werd aangetoond dat zonder extra dataverwerking VOG inderdaad saccade piek snelheden flink kan onderschatten. Saccadische oogbewegingen met een amplitude van minstens 5 ${ }^{\circ}$ hebben echter in goede benadering een beperkte bandbreedte tot ongeveer $25-30 \mathrm{~Hz}$. Het Nyquist theorema stelt daarom dat 1 . Een bemonsterfrequentie van $50-60 \mathrm{~Hz}$ voldoende is om aliasing te voorkomen en 2 . Een signaal met een hogere bemonsterfrequentie gereconstrueerd kan worden. Door de data te interpoleren naar 1 $\mathrm{kHz}$ werd aangetoond dat afwijkingen van saccade piek snelheden gemiddeld $3 \%$ waren, veel kleiner dan natuurlijke variaties. $50-60 \mathrm{~Hz}$ VOG, met gebruikmaking van het Nyquist bemonster theorema om de data te interpoleren naar $1 \mathrm{kHz}$ is dus geschikt om saccade piek snelheden te bepalen in een standaard klinische toepassing.

Additioneel werden in hoofdstuk 7 1. de semi-invasieve scleral search coil (SSC) techniek, beschouwd als de gouden standaard voor oogbewegingsmetingen, 2. de klinisch veelvuldig toegepaste electro nystagmografie (ENG) methode en 3 . een $50 \mathrm{~Hz}$ VOG systeem, met gebruikmaking van het Nyquist bemonster theorema om de data te interpoleren naar $1 \mathrm{kHz}$ met elkaar vergeleken. Voor het detecteren van de amplituden van saccaden zijn VOG en SSC de voorkeurstechnieken, omdat substantiële drift ENG minder geschikt maakt. Als gevolg van niet-lineariteiten in ENG is deze methode ook niet nauwkeurig in detectie van monoculaire oogbewegingen; het gemiddelde ENG van 2 ogen is daarentegen meer nauwkeurig. VOG en ENG zijn de voorkeurstechnieken voor het meten van oog pieksnelheden vanwege 1. slippen van de SSC lens en 2. vertraging van de oogdynamiek door de aanwezigheid van de SSC lens. Dus lijkt VOG, met gebruikmaking van het Nyquist bemonster theorema om de data te interpoleren naar 1 $\mathrm{kHz}$, vanuit een technische oogpunt de klinische keuze te zijn voor het meten van oogbewegingen. VOG faalt echter om het pupilmidden correct te detecteren in personen met hangende oogleden of problemen met het open houden van de ogen tijdens metingen. De meeste regelmatig gebruikte VOG systemen waarschuwen de laborant niet voor de gemaakte fouten. ENG is veel robuuster en kan - ondanks enkele andere tekortkomingen -oogbewegingen meten in bijna iedere situatie, wat pleit voor ENG als klinische toepassing, in ieder geval als backup techniek. 


\section{Nawoord}

Ruim voor de afronding van dit proefschrift had ik me al voorgenomen geen dankwoord te schrijven. De eenvoudige reden daarvoor is dat er zovelen zijn die een bijdrage geleverd hebben aan de totstandkoming van dit boekje, dat ik altijd iemand zal vergeten. Dat is niet mijn stiel.

Dankbaarheid en respect zijn echter het grootste goed, van zowel mens als dier. Daarom, aan iedereen die mij professioneel en vriendschappelijk gevormd heeft tot de persoon die ik nu ben:

Bedankt voor het evenwicht dat je in mijn leven hebt gebracht!

Merci! 


\section{Curriculum Vitae}
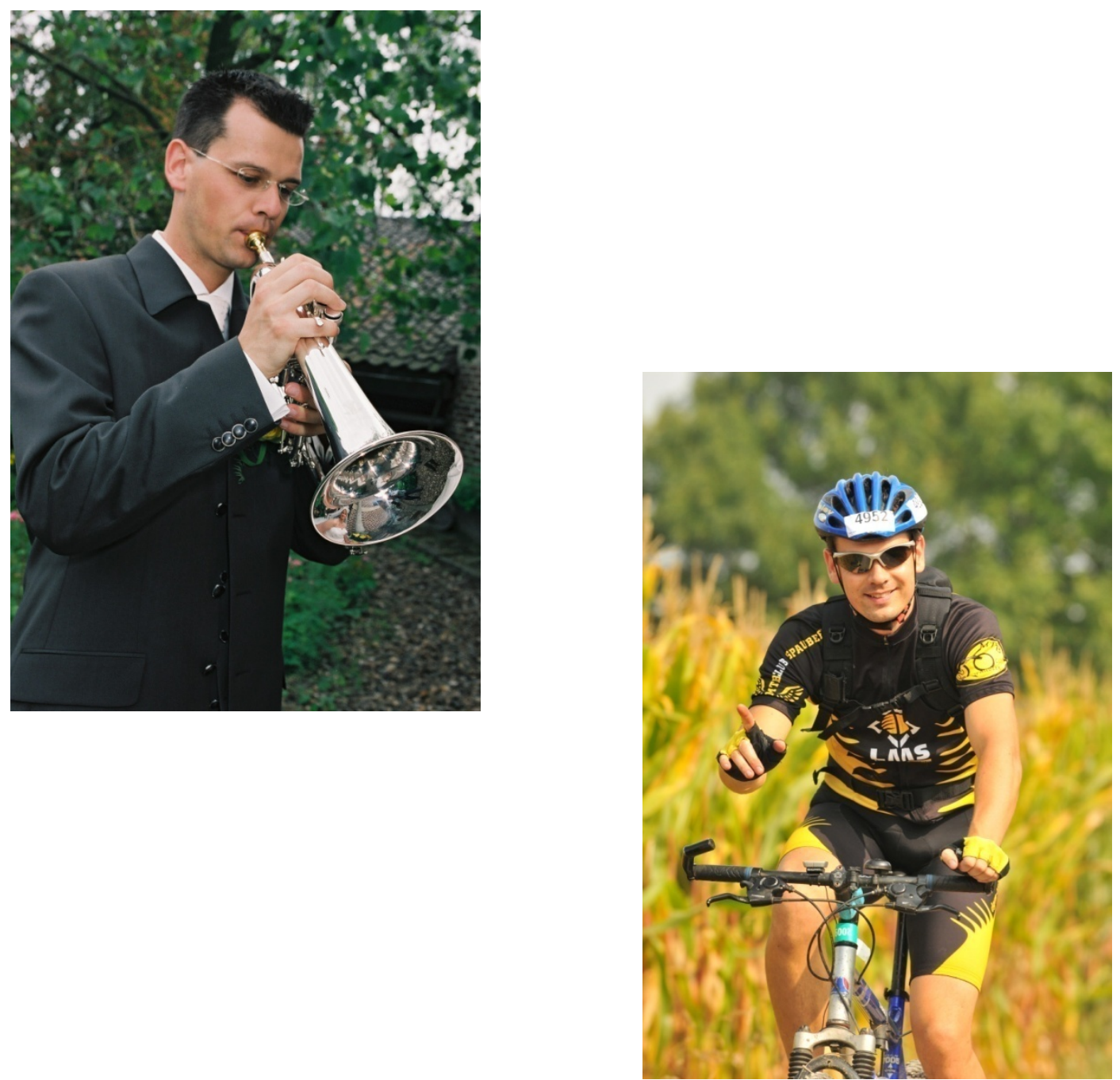
Maurice Janssen is geboren op 29 april 1978 in Heerlen. Hij groeide op in het ZuidLimburgse dorp Neerbeek. In 1996 behaalde hij zijn gymnasium diploma aan de toenmalige scholengemeenschap Sint-Michiel te Geleen. In 1997 verliet hij zijn ouderlijke dorp om Technische Natuurkunde te gaan studeren aan de Technische Universiteit van Eindhoven (TU/e). Dit nadat hij 2 maal was uitgeloot voor geneeskunde en de enige loting ooit voor Biomedische Technologie (BMT). Tijdens zijn studie liep hij stage op het gebied van brachytherapie aan het London Regional Cancer Centre in London, Ontario (Canada). Zijn afstudeerwerk deed hij in het toenmalige Sint Joseph ziekenhuis te Veldhoven op het gebied van diffusie tensor MRI. Onder begeleiding van Carola van Pul en Pieter Wijn rondde hij zijn studie met als afstudeervariant Klinische Fysica af in 2003. Zij interesseerden hem voor de breedte van het vakgebied, en daarom startte hij in 2003 met de opleiding tot Algemeen Klinisch Fysicus in het academisch ziekenhuis Maastricht (azM) onder begeleiding van Jos Reulen. Daartoe verhuisde hij naar Spaubeek, het dorp naast zijn ouderlijk dorp, waar hij ging samenwonen samen met zijn huidige vrouw Nancy. Samen hebben ze 2 prachtige kinderen, Senna en Luca, en een trouwe labrador, Max.

In 2007 behaalde Maurice de registratie Klinisch Fysicus, nadat hij in 2006 zijn opleiding gecombineerd had met een ad interim hoofdschap van de Instrumentele Dienst van het azM. Tijdens zijn opleiding ging zijn interesse vooral uit naar functiemeting en bewaking en focuseerde zijn wetenschappelijke interesse zich op de evenwichtskunde.

$\mathrm{Na}$ zijn opleiding kon hij in het azM blijven om zijn onderwijs- en onderzoekactiviteiten voor de afdeling BMT-azM nog enkele jaren voort te zetten, om zodoende ook zijn wetenschappelijke verdieping te vervolmaken tot dit proefschrift. De verbreding bleef echter lonken, en daarom is hij sinds 2008 ook cursuscoördinator en mentor Klinische Fysica van de School of Medical Physics and Engineering Eindhoven (SMPE/e) en deeltijd Klinisch Fysicus in het Máxima Medisch Centrum.

Maurice besloot in 2010 om niet als a(cademische) Klinisch Fysicus verder te gaan in de evenwichtskunde, maar als A(lgemeen) Klinisch Fysicus in het Orbis Medisch Centrum te Sittard-Geleen. Deze aanstelling combineert hij nu met een detachering bij de SMPE/e. 